CERN-TH/98-303, CTP TAMU-36/98, Imperial/TP/97-98/77,

LPTENS-98/39, UPR/0819-T

hep-th/9810159

October 1998

\title{
M-theory/heterotic Duality: a Kaluza-Klein Perspective
}

\author{
H. Lü ${ }^{\$ \ddagger}$, C.N. Pope ${ }^{\dagger \S 1}$ and K.S. Stelle ${ }^{\star}$ \\ * The Blackett Laboratory, Imperial College ${ }^{2}$ \\ Prince Consort Road, London SW' 2BZ, UK \\ \# TH Division, CERN, CH-1211 Geneva 23, Switzerland \\ ${ }^{\dagger}$ Center for Theoretical Physics, Texas A\&M University, College Station, Texas 77843 \\ \$Department of Physics, University of Pennsylvania, Philadelphia, PA 19104 \\ ${ }^{\ddagger}$ Laboratoire de Physique Théorique de l'École Normale Supérieure ${ }^{2,3}$ \\ 24 Rue Lhomond - 75231 Paris CEDEX 05 \\ $\S_{S I S S A}$, Via Beirut No. 2-4, 34013 Trieste, Italy ${ }^{2}$
}

\begin{abstract}
$\underline{\text { ABSTRACT }}$
We study the duality relationship between M-theory and heterotic string theory at the classical level, emphasising the transformations between the Kaluza-Klein reductions of these two theories on the K3 and $T^{3}$ manifolds. Particular attention is devoted to the corresponding structures of $\sigma$-model cosets and the correspondence between the $p$-brane charge lattices. We also present simple and detailed derivations of the global symmetries and coset structures of the toroidally-compactified heterotic theory in all dimensions $D \geq 3$, making use of the formalism of solvable Lie algebras.
\end{abstract}

\footnotetext{
${ }^{1}$ Research supported in part by DOE grant DE-FG03-95ER40917.

${ }^{2}$ Research supported in part by the EC under TMR contract ERBFMRX-CT96-0045.

${ }^{3}$ Unité Propre du Centre National de la Recherche Scientifique, associée à l'École Normale Supérieure et à l'Université de Paris-Sud.
} 


\section{Contents}

\begin{tabular}{lll}
\hline & Introduction & 2
\end{tabular}

2 Global symmetries of the heterotic string on $T^{n} \quad 3$

$2.1 \quad D=9$ heterotic string $\ldots \ldots \ldots \ldots \ldots \ldots$

2.2 Geometry of $O(p, q) /(O(p) \times O(q))$ cosets $\ldots \ldots \ldots \ldots$. . . . . . 5

2.3 Global $O(10-D, 10-D+N)$ symmetries from dimensional reduction . . . 7

3 Scalar cosets in the $T^{n}$-compactified heterotic theory 9

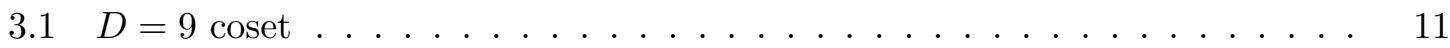

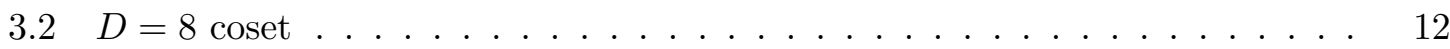

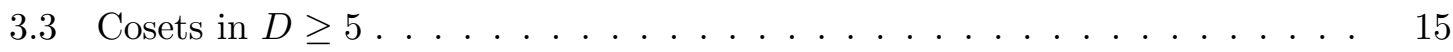

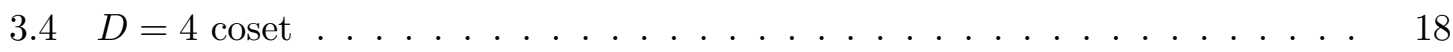

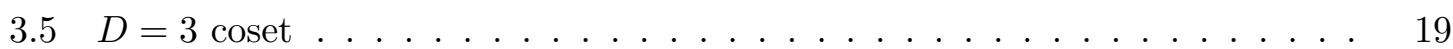

\begin{tabular}{|lll}
4 & Time-like reductions of the heterotic string theory & 21
\end{tabular}

\begin{tabular}{|lll}
5 & K3 compactifications of M-theory & 23
\end{tabular}

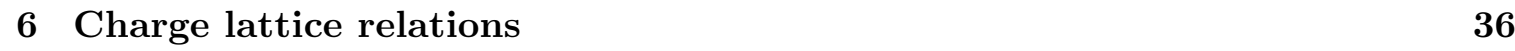

\begin{tabular}{lll}
\hline & $p$-brane orbits with Yang-Mills charges & 40
\end{tabular}

$7.1 \quad D=9$ black holes and Yang-Mills wave excitations . . . . . . . . . . . . . 41

$7.2 \quad 5$-branes in $D=9 \ldots \ldots \ldots \ldots \ldots \ldots \ldots \ldots$

\begin{tabular}{ll}
\hline Appendices & 50
\end{tabular}

\begin{tabular}{|l|l|}
\hline A Toroidal reduction of the heterotic string & 50
\end{tabular}

B Further coset geometry examples

$\begin{array}{ll}\text { References } & 55\end{array}$ 


\section{Introduction}

The duality relations [1, 2, 3, 4] between the heterotic string theory and M-theory are perhaps the most surprising of the web of dualities that is now seen to underpin the search for a satisfactory nonperturbative formulation of a quantum theory of gravity and everything else. One of the most striking features of this web of dualities is the degree to which such nonperturbative relations can be seen already in nonlinear features of the classical or semiclassical field theory limits of the underlying quantum theories. In the present paper, we shall investigate in some detail the classical Kaluza-Klein relations between the heterotic theory and $D=11$ supergravity, which is the field-theory limit of M-theory. Specifically, we shall consider the relation between $D=11$ supergravity compactified on a K3 manifold and the heterotic theory compactified on $T^{3}$.

A number of general features of the M-theory/heterotic correspondence were originally detailed in Refs [1, 2]. In the present paper, we shall study some of the apsects of the Kaluza-Klein reduction procedure in more detail, to reveal further aspects of the correspondences between the two theories. We begin in sections 2 and 3 by considering the nonlinear $\sigma$-model structure of the dimensionally-reduced heterotic string, focusing in particular on the relation between group-theoretic coset descriptions of these $\sigma$-models and embeddings of the $\sigma$-models into linearly realised representations of the numerator symmetry groups, combined with appropriate invariant constraints. Generalising the Borel-subalgebra constructions of analogous $\sigma$-models in the maximally supersymmetric supergravities [5, [6], we find that the appearance of solvable subalgebras [0, 8, 9, 10, 11, 12] of the heterotic theory duality symmetries significantly simplifies the derivation of explicit parametrisations for the corresponding heterotic $\sigma$-model cosets. We include a relatively digestible discussion of solvable Lie algebras, taking examples from some of the simpler global symmetry groups arising in the heterotic compactifications to illustrate the essential ideas.

In section 5, we analyse the dimensional reduction of M-theory on a K3 manifold, using in particular the approximate description of a $\mathrm{K} 3$ manifold as $T^{4} / Z_{2}$, with the 16 orbifold singularities of this construction blown up by the cutting and pasting in of 16 EguchiHanson instantons [13]. This analysis will allow us to establish a detailed correspondence between the fields of M-theory and the heterotic theory, and in particular to establish the relationship between the couplings of dilatonic scalars in the two theories.

In section 6, we proceed to establish the correspondence between the lattices of $p$ brane charges in the two theories. This correspondence reconfirms the structure of the M-theory charge lattice that had originally been derived using duality relations [14, 15] and 
the special properties of "scale-setting" $p$-brane species [16]. The paper concludes with a discussion of the charge orbits containing $p$-brane solutions supported purely by the YangMills sector. Given the currently anticipated general relationship between string theory states and semiclassical solutions, such solutions are required to correspond to the short massive multiplets of Yang-Mills sector states associated to fields in the heterotic Lagrangian acquiring masses from the Higgs effect for a generic heterotic vacuum. A perhaps unsettling feature of the only available supersymmetric $p$-branes supported by the Yang-Mills sector is that they have naked singularities. We shall explain how these naked singularities appear as an artefact of dimensional reduction from wave-like solutions using singular Killing vectors.

In the appendices, we give some details of the toroidal dimensional reduction of the heterotic theory and also some additional embeddings of coset models into constrained linear realisations of symmetry groups.

\section{Global symmetries of the heterotic string on $T^{n}$}

\section{1 $D=9$ heterotic string}

Taking the general $T^{n}$ reduction of the heterotic theory given in Appendix A, and specialising to the case of reduction to $D=9$ on a single circle, we find, after rotating the dilatonic scalars so that

$$
\phi_{1}=\frac{1}{2 \sqrt{2}} \varphi-\frac{1}{2} \sqrt{\frac{7}{2}} \phi, \quad \phi_{2}=\frac{1}{2} \sqrt{\frac{7}{2}} \varphi+\frac{1}{2 \sqrt{2}} \phi,
$$

that the nine-dimensional Lagrangian is given by

$$
\begin{aligned}
e^{-1} \mathcal{L}_{9}= & R-\frac{1}{2}(\partial \phi)^{2}-\frac{1}{2}(\partial \varphi)^{2}-\frac{1}{2} e^{\sqrt{2} \varphi} \sum_{I}\left(\partial B_{(0)}^{I}\right)^{2}-\frac{1}{12} e^{-\sqrt{\frac{8}{7}} \phi}\left(F_{(3)}\right)^{2} \\
& -\frac{1}{4} e^{-\sqrt{\frac{2}{7}} \phi}\left(e^{\sqrt{2} \varphi}\left(F_{(2)}\right)^{2}+e^{-\sqrt{2} \varphi}\left(\mathcal{F}_{(2)}\right)^{2}+\sum_{I}\left(G_{(2)}^{I}\right)^{2}\right)
\end{aligned}
$$

where the $I$ index labels the 16 unbroken Cartan-subalgebra gauge-group generators for a generic "fully-Higgsed" vacuum configuration. From (A.10), and dropping the primes on the potentials, the field strengths are given by

$$
\begin{aligned}
& F_{(3)}=d A_{(2)}+\frac{1}{2} B_{(1)}^{I} d B_{(1)}^{I}-\frac{1}{2} \mathcal{A}_{(1)} d A_{(1)}-\frac{1}{2} A_{(1)} d \mathcal{A}_{(1)}, \\
& \mathcal{F}_{(2)}=d \mathcal{A}_{(1)}, \quad G_{(2)}^{I}=d B_{(1)}^{I}+B_{(0)}^{I} \mathcal{A}_{(1)}, \\
& F_{(2)}=d A_{(1)}+B_{(0)}^{I} d B_{(1)}^{I}+\frac{1}{2} B_{(0)}^{I} B_{(0)}^{I} d \mathcal{A}_{(1)},
\end{aligned}
$$

the nine-dimensional string coupling constant is given by $\lambda_{9}=e^{\sqrt{7 / 8} \phi}$. 
The symmetry group of the scalar manifold, parametrised by the dilatons $\phi$ and $\varphi$, and the axions $B_{(0)}^{I}$, is easily analysed. Let us, for greater generality, consider the case where there are $N$ abelian vectors potentials $B_{(1)}^{I}(1 \leq I \leq N)$ in $D=10$, rather than the particular case $N=16$ arising in the heterotic string. We now introduce the set of $N+2$ fields $X, Y, Z^{I}$ in $\mathbb{R}^{1, N+1}$, defined by

$$
X+Y=e^{\frac{1}{\sqrt{2}} \varphi}, \quad X-Y=e^{-\frac{1}{\sqrt{2}} \varphi}+\frac{1}{2} B_{(0)}^{I} B_{(0)}^{I} e^{\frac{1}{\sqrt{2}} \varphi}, \quad Z^{I}=\frac{1}{\sqrt{2}} B_{(0)}^{I} e^{\frac{1}{\sqrt{2}} \varphi} .
$$

It is evident that these satisfy the constraint

$$
X^{2}-Y^{2}-Z^{I} Z^{I}=1
$$

and that the scalar part of the Lagrangian (2.2) can be written as

$$
e^{-1} \mathcal{L}_{\text {scalar }}=(\partial X)^{2}-(\partial Y)^{2}-\left(\partial Z^{I}\right)^{2}-\frac{1}{2}(\partial \phi)^{2}
$$

subject to the constraint (2.5). Thus we see that the Lagrangian and the constraint are invariant under global $O(1, N+1)$ transformations, which act by matrix multiplication on the column vector $\left(X, Y, Z^{I}\right)$, and also that the Lagrangian is invariant under constant shifts of $\phi$. Thus the symmetry of the scalar Lagrangian is $O(1, N+1) \times \mathbb{R}$.

We find that in terms of the fields $\left(X, Y, Z^{I}\right)$, the Lagrangian (2.2) can be written as

$$
\begin{aligned}
e^{-1} \mathcal{L}_{9}= & R+\mathcal{L}_{\text {scalar }}-\frac{1}{2} e^{-\sqrt{\frac{8}{7}} \phi}\left(F_{(3)}\right)^{2} \\
& +\frac{1}{4} e^{-\sqrt{\frac{2}{7}} \phi}\left(\left(d A_{X}\right)^{2}-\left(d A_{Y}\right)^{2}-\left(d B_{(1)}^{I}\right)^{2}-2\left(X d A_{X}+Y d A_{Y}+Z^{J} d B_{(1)}^{J}\right)^{2}\right),
\end{aligned}
$$

where we define

$$
A_{X}=\frac{1}{\sqrt{2}}\left(A_{(1)}+\mathcal{A}_{(1)}\right), \quad A_{Y}=\frac{1}{\sqrt{2}}\left(A_{(1)}-\mathcal{A}_{(1)}\right)
$$

In terms of these redefined potentials, the 3 -form field $F_{(3)}$ becomes

$$
F_{(3)}=d A_{(2)}-\frac{1}{2} A_{X} d A_{X}+\frac{1}{2} A_{Y} d A_{Y}+\frac{1}{2} B_{(1)}^{I} d B_{(1)}^{I}
$$

It is now manifest that if the $O(1, N+1)$ transformations act on the column co-vector $\left(A_{X}, A_{Y}, B_{(1)}^{I}\right)$ of 1 -form potentials at the same time as they act on $\left(X, Y, Z^{I}\right)$, then the entire Lagrangian (2.7) is invariant. To be precise, if $\Lambda$ is an $O(1, N+1)$ group element then the Lagrangian is invariant under the global transformation

$$
\left(\begin{array}{c}
X \\
Y \\
Z^{I}
\end{array}\right) \longrightarrow \Lambda\left(\begin{array}{c}
X \\
Y \\
Z^{I}
\end{array}\right), \quad\left(\begin{array}{c}
A_{X} \\
A_{Y} \\
B^{I}
\end{array}\right) \longrightarrow\left(\Lambda^{\mathrm{T}}\right)^{-1}\left(\begin{array}{c}
A_{X} \\
A_{Y} \\
B^{I}
\end{array}\right)
$$


Note that $F_{(3)}$ is a singlet. In particular, this completes our demonstration that the dimensional reduction of the heterotic theory with $U(1)^{16}$ gauge fields gives a theory with an $O(1,17)$ global symmetry in $D=9$. There is in addition the previously-mentioned $\mathbb{R}$ factor also, corresponding to a constant shift of the dilaton $\phi$, accompanied by appropriate rescalings of the potentials.

\subsection{Geometry of $O(p, q) /(O(p) \times O(q))$ cosets}

In the previous subsection, we showed how the global symmetry of the heterotic theory when reduced on $S^{1}$ could be understood from a geometrical point of view. In fact, more generally, we showed that the reduction of the bosonic sector of ten-dimensional $N=1$ supergravity coupled to $N$ abelian gauge fields gives rise to a nine-dimensional theory with an $O(1, N+1) \times \mathbb{R}$ global symmetry. The $O(1, N+1)$ symmetry could be understood geometrically as a set of linear transformations on an $(N+2)$-vector of coordinates $\left(X, Y, Z^{I}\right)$ on $\mathbb{R}^{1, N+1}$ with the Minkowski metric $\eta=\operatorname{diag}(-1,1,1, \ldots, 1)$. The scalar manifold is therefore the coset space $O(1, N+1) / O(N+1)$ (together with an extra trivial $\mathbb{R}$ factor).

In this subsection, we shall consider the geometry of the coset spaces that arise in general toroidal dimensional reductions of the heterotic theory. In fact, we shall consider more generally the geometrical construction of the entire class of coset spaces

$$
\frac{O(p, q)}{O(m, q-n) \times O(p-m, n)}
$$

where $0 \leq m \leq p$ and $0 \leq n \leq q$.

To begin, we introduce the indefinite-signature flat metric

$$
\eta=\operatorname{diag}(\underbrace{-1,-1, \ldots,-1}_{p}, \underbrace{1,1, \ldots, 1}_{q}) .
$$

Group elements $W$ in $O(p, q)$ then, by definition, satisfy

$$
W^{\mathrm{T}} \eta W=\eta \text {. }
$$

One can also then impose the additional $O(p, q)$-covariant constraint

$$
W^{\mathrm{T}}=W
$$

Note that matrices $W$ satisfying these two conditions do not form a group; rather, as we shall now show, they decompose into orbits described by cosets of the form (2.11).

To make this more precise, we shall consider the orbits of matrices $W$ satisfying (2.13, 2.14), under the $O(p, q)$ transformations

$$
W \longrightarrow \Lambda^{\mathrm{T}} W \Lambda
$$


Noting that any non-degenerate symmetric matrix $S$ can be diagonalised under the action $S \rightarrow \Lambda^{\mathrm{T}} S \Lambda$ where $\Lambda$ is some $O(p, q)$ matrix, we see that every $O(p, q)$ orbit for $W$ satisfying (2.13, 2.14) passes through a point where $W$ is a diagonal matrix. For diagonal matrices, 2.13) implies directly that the diagonal elements are all \pm 1 . Thus we may characterise the $O(p, q)$ orbits by a fiducial matrix

$$
W_{0}=\operatorname{diag}(\underbrace{-1,-1, \ldots,-1}_{m}, \underbrace{1,1, \ldots, 1}_{p-m}, \underbrace{-1,-1, \ldots,-1}_{n}, \underbrace{1,1, \ldots, 1}_{q-n})
$$

where $0 \leq m \leq p$ and $0 \leq n \leq q$. The specific distributions of the +1 and -1 eigenvalues within the $p \times p$ timelike and $q \times q$ spacelike subspaces may be modified by further $O(p) \times O(q)$ transformations. The fiducial matrix $W_{0}$ in 2.16 is therefore representative of a class of equivalent matrices. Note, however, that no $O(p, q)$ transformation is able to exchange eigenvalues between the timelike and spacelike sectors, since the corresponding eigenvectors would also have to be exchanged, and this is impossible since $O(p, q)$ transformations preserve the norms of vectors, while timelike and spacelike vectors have norms of opposite signs. Thus, the numbers $m$ and $n$ in (2.16) are $O(p, q)$-invariant. These numbers will determine the denominator groups $K$ in the coset spaces $O(p, q) / K$.

For a given fiducial matrix $W_{0}$, the denominator group $K$ is easily determined, since it is nothing but the stability subgroup of $O(p, q)$ that leaves $W_{0}$ invariant. From (2.12) and (2.16), we can see that $W_{0}$ will be left invariant by the subgroup

$$
K=O(m, q-n) \times O(p-m, n)
$$

of $O(p, q)$. Thus the orbit for this particular fiducial matrix $W_{0}$ is the coset space (2.11). Note that all points on the given orbit have matrices $W$ that satisfy the trace condition

$$
\operatorname{tr}(W \eta)=q-p+2(m-n)
$$

This follows from the fact that $\operatorname{tr}(W \eta)$ is manifestly $O(p, q)$-invariant, and that $\operatorname{tr}\left(W_{0} \eta\right)$ can be determined by inspection from (2.12) and (2.16). Note that if we consider the particular fiducial matrix

$$
W_{0}=\operatorname{diag}(1,1, \ldots, 1),
$$

corresponding to $m=n=0$, then the $O(p, q)$ orbits will describe the coset

$$
\frac{O(p, q)}{O(p) \times O(q)} \text {. }
$$

It is cosets of this type that will be principally relevant in our subsequent discussion. We discuss some further examples of classes of cosets in Appendix B. 


\subsection{Global $O(10-D, 10-D+N)$ symmetries from dimensional reduction}

We saw in subsection 2.1 that the bosonic sector of ten-dimensional simple supergravity coupled to $N$ abelian gauge multiplets gives rise, when reduced on $S^{1}$, to a theory with a global $O(1, N+1) \times \mathbb{R}$ symmetry in nine-dimensions. In order to bring out the geometrical structure, we have exploited the fact that the coset $O(1, N+1) / O(N+1)$ could be viewed as a hypersurface in the flat Minkowski-signature space $\mathbb{R}^{1, N+1}$. In this subsection, we shall extend the discussion to the lower-dimensional theories obtained by compactifying on $T^{10-D}$ instead. In these cases, one expects the global symmetry group to be $O(10-D, 10-$ $D+N) \times \mathbb{R}$ when $D \geq 5$, while in $D=4$ and $D=3$ one expects $O(6, N+6) \times S L(2, \mathbb{R})$ and $O(8, N+8)$ respectively. Here, we shall make use of the more general discussion of coset spaces given in subsection 2.2 in order to give a geometrical interpretation of the symmetries of the $D$-dimensional theories.

Our starting point is the $D$-dimensional Lagrangian (A.6), obtained by dimensional reduction from $D=10$. We use the expressions (A.10) for the 3-form and 2-form field strengths, which were obtained after making the field redefinitions (A.9). First, we note that the particular linear combination $\vec{a}_{1} \cdot \vec{\phi}$ of dilatons that couples to the 3 -form field strength is decoupled from all the axionic scalars $A_{(0) \alpha \beta}, \mathcal{A}_{(0) \beta}^{\alpha}$ and $B_{(0) \alpha}^{I}$. In other words, the dot products $\vec{a}_{1} \cdot \vec{a}_{1 \alpha \beta}, \vec{a}_{1} \cdot \vec{b}_{\alpha \beta}$ and $\vec{a}_{1} \cdot \vec{c}_{\alpha}$ all vanish. It is therefore natural to perform a rotation on the dilatons so that the combination $\phi=-\sqrt{(D-2) / 8} \vec{a}_{1} \cdot \vec{\phi}$ is separated from the rest. After this rotation, one expects that the Lagrangian should be expressible in the form

$e^{-1} \mathcal{L}_{D}=R-\frac{1}{2}(\partial \phi)^{2}+\frac{1}{4} \operatorname{tr}\left(\partial \mathcal{M}^{-1} \partial \mathcal{M}\right)-\frac{1}{12} e^{-\sqrt{8 /(D-2)} \phi} F_{(3)}^{2}-\frac{1}{4} e^{-\sqrt{2 /(D-2)} \phi} H_{(2)}^{\mathrm{T}} \mathcal{M} H_{(2)}$,

where $\mathcal{M}$ is a square matrix of dimension $(20-2 D+N)$ that is parametrised by the rest of the dilatonic scalars and the axionic scalars, and $H_{(2)}=d C_{(1)}$ is a column vector formed from the exterior derivatives of the 1 -form potentials. The NS-NS 3 -form $F_{(3)}$ is coupled only to the dilaton $\phi$, implying that the $D$-dimensional string coupling is $\lambda_{D}=\exp (\sqrt{(D-2) / 8} \phi)$.

To show that the Lagrangian (A.6) is invariant under $O(10-D, 10-D+N)$, and that its scalar manifold is the coset space $O(10-D, 10-D+N) /(O(10-D) \times O(10-D+N))$ (together with a further $\mathbb{R}$ factor for the decoupled scalar $\phi$ ), we need only show that it can indeed be written in the form (2.21), and that $\mathcal{M}$ satisfies the constraints (2.13, 2.14) and

\footnotetext{
${ }^{1}$ These symmetry enlargements in $D=4$ and $D=3$ result from dualising the 2-form or 1-form potentials to give additional axionic scalars. If one leaves them undualised instead, then the $D \geq 5$ discussion of global symmetries extends uniformly to include these dimensions too.
} 
it is equal to the identity for some special set of values of the scalar fields. Furthermore, the number of independent scalar fields in $\mathcal{M}$ is equal to the dimension of the coset. The conditions (2.13, 2.14) ensure that the symmetry is $O(10-D, 10-D+N)$, while the occurrence of the special point $\mathcal{M}=\mathbb{1}$ ensures that the orbits are those containing the fiducial point $W_{0}$ given in $(2.20)$, leading to the coset structure 2.20$)$.

The easiest way to determine the $\mathcal{M}$ matrix from (A.6) is by studying the kinetic terms for the 2-form field strengths, and comparing them with the expression $H^{\mathrm{T}} \mathcal{M} H$ in (2.21). From (A.6), we see that, after making the rotation of dilatons described above, we must have

$$
H_{(2)}^{\mathrm{T}} \mathcal{M} H_{(2)}=\sum_{\alpha} e^{\vec{c}_{\alpha} \cdot \vec{\phi}}\left(F_{(2) \alpha}\right)^{2}+\sum_{\alpha} e^{-\vec{c}_{\alpha} \cdot \vec{\phi}}\left(\mathcal{F}_{(2)}^{\alpha}\right)^{2}+\sum_{I}\left(G_{(2)}^{I}\right)^{2},
$$

since we have $\vec{c}_{\alpha}=\vec{a}_{1 \alpha}-\frac{1}{2} \vec{a}_{1}=-\vec{b}_{\alpha}+\frac{1}{2} \vec{a}_{1}$. It is convenient to express the matrix $\mathcal{M}$, which should be symmetric, as $\mathcal{M}=\mathcal{V}^{\mathrm{T}} \mathcal{V}$, so that the left-hand side of 2.22) can be written as $\left(\mathcal{V} H_{(2)}\right)^{\mathrm{T}}\left(\mathcal{V} H_{(2)}\right)$. We can then read off the matrix $\mathcal{V}$ by inspecting the expressions in (A.10) for the 2-form field strengths. It is convenient to order the various 1-form potentials so that the column vector $C_{(1)}$ is given by

$$
C_{(1)}=\left(\begin{array}{c}
A_{(1) \alpha} \\
B_{(1)}^{I} \\
\mathcal{A}_{(1)}^{\alpha}
\end{array}\right), \quad H_{(2)}=d C_{(1)}
$$

where it is understood that the indices $\alpha$ and $I$ increase as one descends downwards through the sets of fields in the column vector. We then find that $\mathcal{V}$ is given by

$$
\mathcal{V}=\left(\begin{array}{c|c|c}
e^{\frac{1}{2} \vec{c}_{\alpha} \cdot \vec{\phi}} \gamma_{\alpha}^{\beta} & e^{\frac{1}{2} \vec{c}_{\alpha} \cdot \vec{\phi}} \gamma_{\alpha}^{\gamma} B_{(0) \gamma}^{I} & e^{\frac{1}{2} \vec{c}_{\alpha} \cdot \vec{\phi}} \gamma_{\alpha}^{\gamma}\left(A_{(0) \gamma \beta}+\frac{1}{2} B_{(0) \gamma}^{I} B_{(0) \beta}^{I}\right) \\
\hline 0 & \delta_{I}^{J} & B_{(0) \alpha}^{I} \\
\hline 0 & 0 & e^{-\frac{1}{2} \vec{c}_{\alpha} \cdot \vec{\phi}} \tilde{\gamma}_{\beta}^{\alpha}
\end{array}\right)
$$

Here, the indices $\alpha$ and $I$ label rows, while $\beta$ and $J$ label columns.

It follows from (A.10) that the 3 -form $F_{(3)}$ can now be written as

$$
F_{(3)}=d A_{(2)}+\frac{1}{2} C_{(1)} \Omega d C_{(1)}
$$

where

$$
\Omega=\left(\begin{array}{c|c|c}
0 & 0 & -\mathbb{1}_{n} \\
\hline 0 & \mathbb{1}_{N} & 0 \\
\hline-\mathbb{1}_{n} & 0 & 0
\end{array}\right)
$$

Here, $\mathbb{1}_{m}$ denotes the $m \times m$ unit matrix, and $n=10-D$. The matrix $\Omega$ has eigenvalues \pm 1 , of which $(10-D)$ are negative and $(10-D+N)$ are positive. In fact $\Omega$ is nothing but 
a metric on the indefinite-signature flat space $\mathbb{R}^{10-D, 10-D+N}$. It is straightforward to see that $\mathcal{V}$ satisfies

$$
\mathcal{V}^{\mathrm{T}} \Omega \mathcal{V}=\Omega
$$

This implies that $\mathcal{V}$ lies in the group $O(10-D, 10-D+N)$. It follows that $\mathcal{M}=\mathcal{V}^{\mathrm{T}} \mathcal{V}$ satisfies the two conditions given in (2.13, 2.14). Furthermore, it is evident from (2.24) that if one sets all the axions and dilatons to zero, then $\mathcal{V}$, and hence $\mathcal{M}$, becomes the identity. Thus, from the discussion at the end of subsection 2.2, it follows that $\mathcal{V}$ and $\mathcal{M}$ give parameterisations of the coset $O(10-D, 10-D+N) /(O(10-D) \times O(10-D+N))$. (Indeed, the number of independent scalar fields in $\mathcal{V}$ is equal to the dimension of the coset.) Note that $F_{(3)}$ as given in (2.25) is a singlet under the $O(10-D, 10-D+N)$ transformations.

In principle, the proof that the $D$-dimensional Lagrangian (A.6) can be written in the form (2.21) could be completed by directly evaluating $\frac{1}{4} \operatorname{tr}\left(\partial \mathcal{M}^{-1} \partial \mathcal{M}\right)$, where $\mathcal{M}=\mathcal{V}^{\mathrm{T}} \mathcal{V}$ and $\mathcal{V}$ is given by (2.24), and showing that it correctly reproduces the terms in the scalar sector of (A.6). However, some more insight into the structure of the theory can be obtained by following a slightly different approach, showing first that $\mathcal{V}$ can be written as the exponential of a Lie algebra. Specifically, this algebra is the solvable Lie subalgebra of the group $O(10-D, 10-D+N)$. It is to this topic that we shall turn in the next section, where we shall be able to complete the proof that the bosonic Lagrangians have $O(10-D, 10-D+N)$ global symmetries.

\section{Scalar cosets in the $T^{n}$-compactified heterotic theory}

The construction of the cosets describing the scalar manifolds arising in the toroidal reductions of eleven-dimensional supergravity have been discussed in detail in [5]. It was shown that the scalar-field coset in $D$-dimensional maximal supergravity can be parametrised by the Borel subalgebra of $E_{n}$, where $n=11-D$. In other words, there is a one-to-one correspondence between the scalar fields in the theory and the generators of the Borel subgroup. This shows that the scalar manifold is the coset $E_{n} / K\left(E_{n}\right)$, where $K\left(E_{n}\right)$ is the maximal compact subalgebra of $E_{n}$, and also shows that the group $E_{n}$ is of the maximally non-compact form $E_{n(+n)}$ [5]. This latter feature is a consequence of the fact that only the maximally non-compact form of a group allows an Iwasawa decomposition into the product of its maximal compact subgroup and its Borel subgroup. Although there are other ways to parameterise the scalar-field cosets, the Borel parameterisation is a particularly convenient one in this context because it is the one that arises naturally in the "step-by-step" 
dimensional reduction procedure.

In the toroidal reduction of the heterotic theory, one expects [17] the global symmetry group in $D$ dimensions to be $O(n, n+16) \times \mathbb{R}$, where $D=10-n>4$. In $D=4$ the symmetry actually enlarges to $O(6,22) \times S L(2, \mathbb{R})[18,19,20,21]$, and in $D=3$ it enlarges to $O(8,24)$ [22,23]. In all of these cases, the symmetry group is not maximally non-compact, and hence a slightly different approach is necessary in order to parameterise the relevant scalar cosets $O(p, q) /(O(p) \times O(q))$. This difference is reflected in the fact that the number of scalar fields is smaller than the dimension of the Borel subgroup of the relevant $O(p, q)$ numerator group.

It is nonetheless convenient, in the context of dimensional reduction, to parameterise the scalar cosets in an analogous manner. The necessary generalisation of the Borel parameterisation is provided by the Iwasawa decomposition [7]. This decomposition is rather more subtle in the case of groups that are not maximally non-compact. One again has a unique factorisation of a group element $g \in G$ into a product $g=k a n$, where $k$ is in the maximal compact subgroup $K, a$ is in the maximal non-compact Abelian subgroup $A$, and $n$ is in the nilpotent subgroup $N$ of $G$. (In the case where $G$ is maximally non-compact, $A$ is the entire Cartan subgroup and $N$ is the strict Borel subgroup, so the product $A N$ belongs to the standard Borel subgroup.) At the level of the algebra, the Iwasawa decomposition implies that

$$
G=K \oplus G_{s}
$$

where $K$ denotes the generators of the maximal compact subalgebra of $G$, and $G_{s}$ is a so-called Solvable Lie Algebra, comprising a subset of the Borel generators of $G$. To be specific, it comprises the non-compact Cartan generators $H^{\mathrm{nc}}$, together with the subset of the positive-root generators that has strictly positive weights under $H^{\text {nc }}$. (Clearly if $G$ were maximally non-compact, in which case all the Cartan generators would be non-compact, $G_{s}$ would comprise the entire Borel subalgebra.)

The mathematical understanding of solvable Lie algebras relevant to supergravity stems from Ref. [8]. The application of solvable Lie algebras has been extensively studied recently in [9, 10, 11, 12]. The exponential $\mathcal{V}=\exp \left(G_{s}\right)$ gives a parameterisation of the coset $G / K$. From this, one can construct the $G$-invariant scalar coset Lagrangian

$$
\begin{aligned}
e^{-1} \mathcal{L}_{\text {scalar }} & =\frac{1}{4} \operatorname{tr}\left(\partial_{\mu} \mathcal{M}^{-1} \partial^{\mu} \mathcal{M}\right) \\
& =-\frac{1}{2} \operatorname{tr}\left[\partial \mathcal{V} \mathcal{V}^{-1}\left(\partial \mathcal{V} \mathcal{V}^{-1}+\left(\partial \mathcal{V} \mathcal{V}^{-1}\right) \#\right)\right]
\end{aligned}
$$

where $\mathcal{M}=\mathcal{V}^{\#} \mathcal{V}$. Here $\mathcal{V}^{\#}=\tau\left(\mathcal{V}^{-1}\right)$ where $\tau$ denotes the Cartan involution which reverses 
the sign of all the non-compact generators, while leaving the sign of the compact generators unchanged (see, for example, [5]). (For orthogonal groups, $\mathcal{V}^{\#}$ is just equal to the transpose $\mathcal{V}^{\mathrm{T}}$, and for unitary groups it is the Hermitean conjugate $\mathcal{V}^{\dagger}$.) The Lagrangian is invariant under the global symmetry transformations $\mathcal{V} \longrightarrow \mathcal{V}^{\prime}=\mathcal{O} \mathcal{V} \Lambda$, where $\Lambda$ is any element of the group $G$, and $\mathcal{O}$ is a field-dependent compensating transformation that is used to bring the transformed coset representative $\mathcal{V}^{\prime}$ back to the form $\mathcal{V}^{\prime}=\exp \left(G_{s}^{\prime}\right)$. The Iwasawa decomposition guarantees the existence of $\mathcal{O}$, and the fact that it is contained in the maximal compact subgroup $K$. It then follows that $\mathcal{M}$ is transformed to $\mathcal{M}^{\prime}=\Lambda^{\#} \mathcal{M} \Lambda$, and hence that the Lagrangian (3.2) is invariant.

In this section, we shall show that the scalar Lagrangians for the toroidally-reduced het-

erotic theory can be written in the form (3.2), where $\mathcal{V} \sim e^{\frac{1}{2} \vec{\phi} \cdot \vec{H}+\chi_{a} E^{a}}$. We shall obtain the explicit forms of the algebras for the generators $\vec{H}$ and $E^{a}$, and we shall show that they are the solvable Lie algebras associated with the global symmetry groups. This provides an explicit derivation of the global symmetries of the scalar sectors of the toroidally-compactified heterotic theory. We show that the $\mathcal{M}$ obtained from the scalar sector using the Solvable Lie Algebra technique and the one obtained by studying the coupling of the scalars with the vector potentials are equivalent, hence completing the proof that the full Lagrangian has an $O(10-D, 10-D+N)$ global symmetry.

\section{1 $D=9$ coset}

In $D=9$ it is easy to see how to write the scalar sector of the Lagrangian (2.2) in a coset formulation. Let us, for this purpose, omit $\phi$, since it decouples from the rest of the scalars, and plays no significant rôle in the discussion. We introduce generator matrices $H$ and $E_{I}$, associated with the scalars $\varphi$ and $B_{(0)}^{I}$ respectively, and we define the coset representative

$$
\mathcal{V}=e^{\frac{1}{2} \varphi H} e^{B_{(0)}^{I} E_{I}} .
$$

The scalar Lagrangian for $\varphi$ and $B_{(0)}^{I}$ can then be written as

$$
\mathcal{L}=\frac{1}{4} \operatorname{tr}\left(\partial_{\mu} \mathcal{M}^{-1} \partial^{\mu} \mathcal{M}\right), \quad \text { where } \quad \mathcal{M}=\mathcal{V}^{\mathrm{T}} \mathcal{V}
$$

provided that the generators $H$ and $E_{I}$ satisfy the algebra

$$
\left[H, E_{I}\right]=\sqrt{2} E_{I}, \quad[H, H]=0 \quad\left[E_{I}, E_{J}\right]=0 .
$$

This is a subalgebra of $O(1, N+1)$. To see this, we first need to establish conventions and notation for the generators and roots of the orthogonal algebras. 
The orthogonal algebras $O(p, q)$ divide into two cases, namely the $D_{n}$ series when $p+q=$ $2 n$, and the $B_{n}$ series when $p+q=2 n+1$. The positive roots are given in terms of an orthonormal basis $e_{i}$ as follows:

$$
\begin{array}{ll}
D_{n}: & e_{i} \pm e_{j}, \quad i<j \leq n, \\
B_{n}: & e_{i} \pm e_{j}, \quad i<j \leq n, \quad \text { and } \quad e_{i},
\end{array}
$$

where $e_{i} \cdot e_{j}=\delta_{i j}$. It is sometimes convenient to take $e_{i}$ to be the $n$-component vector $e_{i}=(0,0, \ldots, 0,1,0, \ldots, 0)$, where the " 1 " component occurs at the $i$ 'th position. However, we shall find later that a different basis is more suitable for our purposes. The Cartan subalgebra generators, specified in a basis-independent fashion, are $h_{e_{i}}$, which satisfy $\left[h_{e_{i}}, E_{e_{j} \pm e_{k}}\right]=\left(\delta_{i j} \pm \delta_{i k}\right) E_{e_{j} \pm e_{k}}$, etc. Of these, $\min (p, q)$ are non-compact, with the remainder being compact. It is convenient to take the non-compact ones to be $h_{e_{i}}$ with $1 \leq i \leq \min (p, q)$.

Returning now to our algebra (3.5), we find that the generators $H$ and $E_{I}$ can be expressed in terms of the $O(1, N+1)$ basis as follows:

$$
\begin{aligned}
H & =\sqrt{2} h_{e_{1}}, \\
E_{2 k-1} & =E_{e_{1}-e_{2 k}}, \quad E_{2 k}=E_{e_{1}+e_{2 k}} \quad 1 \leq k \leq\left[\frac{1}{2}+\frac{1}{4} N\right], \\
E_{1+\frac{1}{2} N} & =E_{e_{1}}, \quad \text { if } N \text { is even } .
\end{aligned}
$$

It is easily seen that $h_{e_{1}}$ and $E_{e_{1} \pm e_{i}}$, together with $E_{e_{1}}$ in the case of $N$ even, are precisely the generators of the solvable Lie algebra of $O(1, N+1)$. In other words, $h_{e_{1}}$ is the noncompact Cartan generator of $O(1, N+1)$, while the other generators in (3.7) are precisely the subset of positive-root $O(1, N+1)$ generators that have strictly positive weights under $h_{e_{1}}$. Thus it follows from the general discussion at the beginning of this section that the scalar Lagrangian for the $D=9$ theory is described by the coset $(O(1, N+1) / O(N+1)) \times \mathbb{R}$. (Recall that there is the additional decoupled scalar field $\phi$ with an $\mathbb{R}$ shift symmetry.)

\section{$3.2 \quad D=8$ coset}

Turning now to the reduction of the heterotic theory to $D=8$, we begin from the general toroidal reduction given in Appendix $\mathrm{A}$, and make the following orthogonal transformation

\footnotetext{
${ }^{2}$ It should be emphasised that the mere fact that one can embed the algebra (3.5) into the Lie algebra of a larger Lie group $G$ does not, of itself, mean that the group $G$ acts effectively on the scalar manifold. Only when (3.5) is the solvable Lie algebra of the group $G$ can one deduce that $G$ has an effective group action on the scalar manifold.
} 
of the dilatons:

$$
\left(\begin{array}{l}
\phi_{1} \\
\phi_{2} \\
\phi_{3}
\end{array}\right)=\left(\begin{array}{ccc}
-\sqrt{\frac{3}{4}} & \sqrt{\frac{1}{8}} & \sqrt{\frac{1}{8}} \\
\sqrt{\frac{3}{28}} & -\sqrt{\frac{1}{56}} & \sqrt{\frac{7}{8}} \\
\sqrt{\frac{1}{7}} & \sqrt{\frac{6}{7}} & 0
\end{array}\right)\left(\begin{array}{l}
\phi \\
\varphi_{1} \\
\varphi_{2}
\end{array}\right) .
$$

In terms of these rotated fields, the Lagrangian for the scalar subsector of the eightdimensional theory becomes

$$
\begin{aligned}
e^{-1} \mathcal{L}_{8}= & -\frac{1}{2}(\partial \phi)^{2}-\frac{1}{2}\left(\partial \varphi_{1}\right)^{2}-\frac{1}{2}\left(\partial \varphi_{2}\right)^{2}-\frac{1}{2} e^{\sqrt{2}\left(\varphi_{1}+\varphi_{2}\right)}\left(\partial A_{(0) 23}+B_{(0) 2}^{I} \partial B_{(0) 3}^{I}\right)^{2} \\
& -\frac{1}{2} e^{\sqrt{2}\left(\varphi_{1}-\varphi_{2}\right)}\left(\partial \mathcal{A}_{(0) 3}^{2}\right)^{2}-\frac{1}{2} e^{\sqrt{2} \varphi_{2}}\left(\partial B_{(0) 2}^{I}\right)^{2}-\frac{1}{2} e^{\sqrt{2} \varphi_{1}}\left(\partial B_{(0) 3}^{I}-\mathcal{A}_{(0) 3}^{2} \partial B_{(0) 2}^{I}\right)^{2}
\end{aligned}
$$

For generality, we again allow the range of the index $I$ to be $1 \leq I \leq N$, rather than just the specific range $1 \leq I \leq 16$ that arises in the heterotic theory. The eight-dimensional string coupling constant is given by $\lambda_{8}=e^{\sqrt{3 / 4} \phi}$. Note that the dilaton $\phi$ decouples from the rest of the scalars. We shall therefore temporarily suppress $\phi$ in the following discussion of the coset structure of the scalar manifold, with the understanding that its constant shift symmetry contributes an additional independent $\mathbb{R}$ factor to the full global symmetry.

We can then show that the Lagrangian (3.9), with $\phi$ omitted, can be obtained by parameterising a coset as

$$
\mathcal{V}=e^{\frac{1}{2} \vec{\varphi} \cdot \vec{H}} e^{\mathcal{A}_{(0) 3}^{2} E_{2}{ }^{3}} e^{A_{(0) 23} V^{23}} e^{B_{(0) 2}^{I} U_{I}^{2}} e^{B_{(0) 3}^{I} U_{I}^{3}}
$$

and substituting this into the first line of $(3.2)$, with $\mathcal{M}=\mathcal{V}^{\mathrm{T}} \mathcal{V}$. The commutation relations for the various generators can then be read off by noting that the 1-form field strengths are given by [5]

$$
\mathcal{G}=d \mathcal{V} \mathcal{V}^{-1}=\frac{1}{2} d \vec{\varphi} \cdot \vec{H}+\mathcal{F}_{(1) 3}^{2} E_{2}^{3}+F_{(1) 23} V^{23}+G_{(1) 2}^{I} U_{I}^{2}+G_{(1) 3}^{I} U_{I}^{3}
$$

Comparing with the explicit expressions given in Appendix A and in (3.9), we find that

$$
\begin{aligned}
& {\left[H_{1}, V^{23}\right]=\sqrt{2} V^{23}, \quad\left[H_{2}, V^{23}\right]=\sqrt{2} V^{23},} \\
& {\left[H_{1}, E_{2}{ }^{3}\right]=\sqrt{2} E_{2}{ }^{3}, \quad\left[H_{2}, E_{2}{ }^{3}\right]=-\sqrt{2} E_{2}{ }^{3},} \\
& {\left[H_{1}, U_{I}^{2}\right]=0, \quad\left[H_{2}, U_{I}^{2}\right]=\sqrt{2} U_{I}^{2},} \\
& {\left[H_{1}, U_{I}^{3}\right]=\sqrt{2} U_{I}^{3}, \quad\left[H_{2}, U_{I}^{3}\right]=0,} \\
& {\left[U_{I}^{2}, U_{J}^{3}\right]=\delta_{I J} V^{23}, \quad\left[E_{2}^{3}, U_{I}^{2}\right]=-U_{I}^{3},}
\end{aligned}
$$

with all other commutators vanishing.

We shall now show that the algebra (3.12) is precisely the solvable Lie algebra for $O(2, N+2)$ (or, in other words, that the exponentiation of (3.12) gives a parameterisation 
of the coset $O(2, N+2) /(O(2) \times O(N+2)))$. To do this, it is instructive to look first at two examples, namely $O(2,3)$ and $O(2,4)$ corresponding to $N=1$ and $N=2$. From (3.6), the positive roots for $O(2,3)$ are $e_{1}-e_{2}, e_{1}+e_{2}, e_{1}$ and $e_{2}$. On the other hand, from (3.12) we have four positive-root generators in this case, namely $E_{2}{ }^{3}, V^{23}, U_{1}^{2}$ and $U_{1}^{3}$. It is easy to see that the commutation relations in (3.12) lead to the identifications

$$
\begin{array}{ll}
E_{2}{ }^{3}=E_{e_{1}-e_{2}}, & V^{23}=E_{e_{1}+e_{2}}, \quad U_{1}^{2}=E_{e_{2}}, \quad U_{1}^{2}=E_{e_{1}}, \\
H_{1}=\sqrt{2} h_{e_{1}}, & H_{2}=h_{e_{2}} .
\end{array}
$$

Note that in this case, because $O(2,3)$ is maximally non-compact, all of the Borel generators of $O(2,3)$ occur in the associated solvable Lie algebra.

For the case $O(2,4)$, we have generators $E_{2}{ }^{3}, V^{23}, U_{I}^{2}$ and $U_{I}^{3}$ in our coset parameterisation (3.10), where $1 \leq I \leq 2$. From (3.6), the positive roots of $O(2,4)$ are $e_{1} \pm e_{2}, e_{1} \pm e_{3}$ and $e_{2} \pm e_{3}$. From the algebra (3.12), it is clear that we should take

$$
H_{1}=\sqrt{2} h_{e_{1}}, \quad H_{2}=\sqrt{2} h_{e_{2}} \quad E_{2}^{3}=E_{e_{1}-e_{2}}, \quad V^{23}=E_{e_{1}+e_{2}} .
$$

It is then evident that in order for the remaining generators to have the proper weights under $H_{1}$ and $H_{2}$, we must have

$$
\begin{array}{ll}
U_{1}^{2}=\alpha_{1} E_{e_{2}+e_{3}}+\beta_{1} E_{e_{2}-e_{3}}, & U_{1}^{3}=\alpha_{1} E_{e_{1}+e_{3}}+\beta_{1} E_{e_{1}-e_{3}}, \\
U_{2}^{2}=\alpha_{2} E_{e_{2}+e_{3}}+\beta_{2} E_{e_{2}-e_{3}}, & U_{2}^{3}=\alpha_{2} E_{e_{1}+e_{3}}+\beta_{2} E_{e_{1}-e_{3}},
\end{array}
$$

for appropriate constants $\alpha_{1}, \beta_{1}, \alpha_{2}$ and $\beta_{2}$. Choosing our sign conventions for $O(2,4)$ so that

$$
\left[E_{e_{1}-e_{2}}, E_{e_{2} \pm e_{3}}\right]=-E_{e_{1} \pm e_{3}}, \quad\left[E_{e_{1} \pm e_{3}}, E_{e_{2} \mp e_{3}}\right]=-E_{e_{1}+e_{2}},
$$

and bearing in mind that the relations (3.15) should preserve the strengths of the generators, we find that, up to arbitrariness in the phases, the solution is $\alpha_{1}=\beta_{1}=1 / \sqrt{2}, \alpha_{2}=-\beta_{2}=$ $\mathrm{i} / \sqrt{2}$. Thus we have

$$
\begin{array}{ll}
U_{1}^{2}=\frac{1}{\sqrt{2}}\left(E_{e_{2}+e_{3}}+E_{e_{2}-e_{3}}\right), & U_{1}^{3}=\frac{1}{\sqrt{2}}\left(E_{e_{1}+e_{3}}+E_{e_{1}-e_{3}}\right), \\
U_{2}^{2}=\frac{\mathrm{i}}{\sqrt{2}}\left(E_{e_{2}+e_{3}}-E_{e_{2}-e_{3}}\right), & U_{2}^{3}=\frac{\mathrm{i}}{\sqrt{2}}\left(E_{e_{1}+e_{3}}-E_{e_{1}-e_{3}}\right),
\end{array}
$$

It is now straightforward to generalise the result to the generic case $O(2, N+2)$. The new feature that we have seen for $N=2$, where the generators for the Yang-Mills axions are expressed in terms of certain real or imaginary combinations of the $O(2,4)$ generators, 
persists for all $N \geq 2$. We find that the embedding of the generators in (3.10) into $O(2, N+2)$ is as follows. When $N$ is even, we find

$$
\begin{array}{lc}
H_{1}=\sqrt{2} h_{e_{1}}, \quad H_{2}=\sqrt{2} h_{e_{2}}, & E_{2}{ }^{3}=E_{e_{1}-e_{2}}, \quad V^{23}=E_{e_{1}+e_{2}}, \\
U_{2 k-1}^{2}=\frac{1}{\sqrt{2}}\left(E_{e_{2}+e_{k+2}}+E_{e_{2}-e_{k+2}}\right), & U_{2 k-1}^{3}=\frac{1}{\sqrt{2}}\left(E_{e_{1}+e_{k+2}}+E_{e_{1}-e_{k+2}}\right), \\
U_{2 k}^{2}=\frac{\mathrm{i}}{\sqrt{2}}\left(E_{e_{2}+e_{k+2}}-E_{e_{2}-e_{k+2}}\right), & U_{2 k}^{3}=\frac{\mathrm{i}}{\sqrt{2}}\left(E_{e_{1}+e_{k+2}}-E_{e_{1}-e_{k+2}}\right),
\end{array}
$$

where $k$ has the range $1 \leq k \leq\left[\frac{1}{2} N\right]$. If $N$ is odd, in addition to the identifications (3.18) for $1 \leq k \leq\left[\frac{1}{2} N\right]$, we have

$$
U_{N}^{2}=E_{e_{2}}, \quad U_{N}^{3}=E_{e_{1}} .
$$

(This embedding of the generators of the solvable Lie algebra in $O(2, N+2)$ was also encountered in [1].)

It is easy to see that the subset of $O(2, N+2)$ generators $h_{e_{1}}, h_{e_{2}}, E_{e_{1} \pm e_{2}}, E_{e_{1} \pm e_{i}}$ and $E_{e_{2} \pm e_{i}}$, with $3 \leq i \leq 2+\left[\frac{1}{2} N\right]$, together with $E_{e_{1}}$ and $E_{e_{2}}$ if $N$ is odd, precisely constitute the set of generators in the solvable Lie algebra of $O(2, N+2)$. This is because $h_{e_{1}}$ and $h_{e_{2}}$ are the two non-compact Cartan generators of $O(2, N+2)$, and the positive-root generators we have just listed are the full set that have strictly positive weights under $h_{e_{1}}$ and $h_{e_{2}}$. Thus it follows that the quantity $\mathcal{V}$ defined in (3.10) gives a parameterisation of the coset $O(2, N+2) /(O(2) \times O(N+2))$. Together with the shift symmetry of the dilaton $\phi$, this shows that the scalar Lagrangian (3.9) is invariant under global $O(2, N+2) \times \mathbb{R}$ transformations.

\subsection{Cosets in $D \geq 5$}

Having seen how the coset construction works in the special cases in $D=9$ and $D=8$, we are now in a position to consider the general $D$-dimensional case. However, owing to the fact that higher-degree fields can be dualised to give additional scalars in $D=4$ and $D=3$, we shall treat these two dimensions separately, having first considered the more straightforward cases $D \geq 5$.

From Appendix $\mathrm{A}$, the scalar Lagrangian in $D$ dimensions can be expressed as

$$
e^{-1} \mathcal{L}_{D}=-\frac{1}{2}(\partial \vec{\varphi})^{2}-\frac{1}{2} \sum_{i<j} e^{\vec{b}_{i j} \cdot \vec{\varphi}}\left(\mathcal{F}_{(0) j}^{i}\right)^{2}-\frac{1}{2} \sum_{i<j} e^{\vec{a}_{i j} \cdot \vec{\varphi}}\left(F_{(1) i j}\right)^{2}-\frac{1}{2} \sum_{i, I} e^{\vec{c}_{i} \cdot \vec{\varphi}}\left(G_{(1) i}^{I}\right)^{2}
$$

together with a free Lagrangian for the dilaton

$$
\phi=-\sqrt{\frac{D-2}{8}} \vec{a}_{123} \cdot \vec{\phi}
$$

which is decoupled from (3.20). The 3 -form field strength $F_{(3)}$ couples only to $\phi$, and the string coupling constant is given by $\Lambda_{D}=\exp (\sqrt{(D-2) / 8} \phi)$. We shall, as usual, 
concentrate only on the sector with $\phi$ omitted during our discussion of the global symmetries. Note that here the notation for the dilaton vectors used here is a little different from the one introduced in Appendix A. Since the dilaton $\phi$ has been truncated out, the dilaton vectors in (3.20) have $(10-D)$ components rather than $(11-D)$. They are given by

$$
\vec{b}_{i j}=\sqrt{2}\left(-\vec{e}_{i}+\vec{e}_{j}\right), \quad \vec{a}_{i j}=\sqrt{2}\left(\vec{e}_{i}+\vec{e}_{j}\right), \quad \vec{c}_{i}=\sqrt{2} \vec{e}_{i}
$$

We have also changed from indices $\alpha, \beta, \ldots$ which range from 2 to $(11-D)$ to $i, j, \ldots$ which range from 1 to $(10-D)$. Since there will be no confusion, we shall use the same symbols $\vec{b}_{i j}$ and $\vec{c}_{i}$ as in Appendix $\mathrm{A}$, and $\vec{a}_{i j}$ in place of $\vec{a}_{1 \alpha \beta}$. The 1-form field strengths in (3.20) are given by

$$
\begin{aligned}
\mathcal{F}_{(1) j}^{i} & =\gamma^{k}{ }_{j} d \mathcal{A}_{(0) k}^{i}, \\
F_{(1) i j} & =\gamma^{k}{ }_{i} \gamma^{\ell}{ }_{j}\left(d A_{(0) k \ell}+B_{(0)[k}^{I} d B_{(0) \ell]}^{I}\right), \\
G_{(1) i}^{a} & =\gamma^{j}{ }_{i} d B_{(0) j}^{I} .
\end{aligned}
$$

We find that we can write the Lagrangian (3.20) in the form (3.2), where the coset representative $\mathcal{V}$ is parametrised as [5]

$$
\mathcal{V}=e^{\frac{1}{2} \vec{\phi} \cdot \vec{H}} e^{\mathcal{A}_{(0) j}^{i} E_{i}^{j}} e^{\frac{1}{2} A_{(0) i j} V^{i j}} e^{B_{(0) i}^{I} U_{I}^{i}}
$$

The commutation relations for the various generators can be determined by comparing the expression for the field strengths

$$
d \mathcal{V} \mathcal{V}^{-1}=\frac{1}{2} d \vec{\phi} \cdot \vec{H}+\sum_{i<j} e^{\frac{1}{2} \vec{b}_{i j} \cdot \vec{\phi}} \mathcal{F}_{(1) j}^{i} E_{i}^{j}+\sum_{i<j} e^{\frac{1}{2} \vec{a}_{i j} \cdot \vec{\phi}} F_{(1) i j} V^{i j}+\sum_{i, I} e^{\frac{1}{2} \vec{c}_{i} \cdot \vec{\phi}} G_{(1) i}^{I} U_{I}^{i}
$$

with the expressions given in 3.23 . We find that the non-vanishing commutators are given by

$$
\begin{aligned}
& {\left[\vec{H}, E_{i}{ }^{j}\right]=\vec{b}_{i j} E_{i}^{j}, \quad\left[\vec{H}, V^{i j}\right]=\vec{a}_{i j} V^{i j}, \quad\left[\vec{H}, U_{I}{ }^{i}\right]=\vec{c}_{i} U_{I}{ }^{i},} \\
& {\left[E_{i}{ }^{j}, E_{k}^{\ell}\right]=\delta_{k}^{j} E_{i}^{\ell}-\delta_{i}^{\ell} E_{k}{ }^{j},} \\
& {\left[E_{i}{ }^{j}, V^{k \ell}\right]=-\delta_{i}^{k} V^{j \ell}-\delta_{i}^{\ell} V^{k j}, \quad\left[E_{i}{ }^{j}, U_{I}{ }^{k}\right]=-\delta_{i}^{k} U_{I}^{j},} \\
& {\left[U_{I}{ }^{i}, U_{J}{ }^{j}\right]=\delta_{I J} V^{i j} .}
\end{aligned}
$$

The way in which the multiple commutators arising in the evaluation of $d \mathcal{V} \mathcal{V}^{-1}$ conspire to produce the precise expressions (3.23) is discussed in detail in [5, 24].

We shall now show that the above set of generators and their commutation relations can be embedded into those of $O(10-D, 10-D+N)$, and that in fact they precisely 
correspond to the solvable lie algebra of $O(10-D, 10-D+N)$. To see this, it is useful first to introduce the set of orthonormal vectors $\tilde{e}_{i}$, related to $e_{i}$ by

$$
\tilde{e}_{i}=e_{11-D-i}, \quad 1 \leq i \leq 10-D
$$

Let us, for definiteness, first consider the case where $N$ is even. We then find that the above generators can be written in terms of those of $O(10-D, 10-D+N)$ as follows. The generators $\vec{H}, E_{i}{ }^{j}$ and $V^{i j}$ are written as

$$
H_{i}=\sqrt{2} h_{\tilde{e}_{i}}, \quad E_{i}^{j}=E_{-\tilde{e}_{i}+\tilde{e}_{j}}, \quad V^{i j}=E_{\tilde{e}_{i}+\tilde{e}_{j}},
$$

where we have $1 \leq i<j \leq 10-D$. For the generators $U_{I}^{i}$ associated with the Yang-Mills axions, we find that we can write

$$
\begin{aligned}
U_{2 k-1}^{i} & =\frac{1}{\sqrt{2}}\left(E_{\tilde{e}_{i}+e_{k+m}}+E_{\tilde{e}_{i}-e_{k+m}}\right), \\
U_{2 k}^{i} & =\frac{i}{\sqrt{2}}\left(E_{\tilde{e}_{i}+e_{k+m}}-E_{\tilde{e}_{i}-e_{k+m}}\right),
\end{aligned}
$$

where $m=10-D$ and $1 \leq k \leq\left[\frac{1}{2} N\right]$. If $N$ is odd, then in addition we have

$$
U_{N}^{i}=E_{\tilde{e}_{i}}
$$

It is easily seen that this set of generators comprises the solvable Lie algebra of $O(10-$ $D, 10-D+N)$. In other words, they are written in terms of the complete set of non-compact Cartan generators of $O(10-D, 10-D+N)$, together with all the positive-root generators that have strictly positive weights under the non-compact Cartan generators.

So far in this subsection, we have constructed the coset representative $\mathcal{V}$ for the purpose of writing the scalar Lagrangian in the form $\frac{1}{4} \operatorname{tr}\left(\partial \mathcal{M}^{-1} \partial \mathcal{M}\right)$, where $\mathcal{M}=\mathcal{V}^{\mathrm{T}} \mathcal{V}$. Using the solvable Lie algebra formalism, we have accordingly shown that the scalar Lagrangian is described by the coset $O(10-D, 10-D+N) /((O(10-D) \times O(10-D+N))$ (together with an extra $\mathbb{R}$ factor for the scalar field $\phi$ ). This construction is abstract, in the sense that we have not taken any specific realisation for the generators; they are simply required to satisfy (3.26). On the other hand, in section 2 we have also obtained an expression for a coset parameterisation $\mathcal{V}$, by considering the coupling of the scalars to the 1 -form potentials. Since these potentials form a fundamental representation of $O(10-D, 10-D+N)$, the representation for $\mathcal{V}$ that we obtained there was necessarily given in terms of matrices of dimension $(20-2 D+N)$. To complete the proof that the entire $D$-dimensional Lagrangian has a global $O(10-D, 10-D+N)$ symmetry, we need to make contact between the two descriptions, by showing explicitly that we can write $\mathcal{V}$ as given by (2.24) in the form (3.24), and by showing that the generator matrices satisfy the commutation relations (3.26). 
To do this, we need only look at the form of (2.24) in the neighbourhood of the identity, in which case it is easy to read off the generator matrices associated with each of the scalar fields.] By this means, we see that the generators are given as follows:

$$
\begin{aligned}
\vec{H}_{i} & =\left(\begin{array}{c|c|c}
\sum_{i} \vec{c}_{i} e_{i i} & 0 & 0 \\
\hline 0 & 0 & 0 \\
\hline 0 & 0 & -\sum_{i} \vec{c}_{i} e_{i i}
\end{array}\right), \quad E_{i}^{j}=\left(\begin{array}{c|c|c|c}
-e_{j i} & 0 & 0 \\
\hline 0 & 0 & 0 \\
\hline 0 & 0 & e_{i j}
\end{array}\right), \\
V^{i j} & =\left(\begin{array}{c|c|c}
0 & 0 & e_{i j}-e_{j i} \\
\hline 0 & 0 & 0 \\
\hline 0 & 0 & 0
\end{array}\right), \quad U_{I}^{i}=\left(\begin{array}{c|c|c}
0 & e_{i I} & 0 \\
\hline 0 & 0 & e_{I i} \\
\hline 0 & 0 & 0
\end{array}\right) .
\end{aligned}
$$

Here, each $e_{a b}$ is defined to be a matrix of the appropriate dimensions that has zeroes in all its entries except for a 1 in the entry at row $a$ and column $b$. These satisfy the matrix product rule $e_{a b} e_{c d}=\delta_{b c} e_{a d}$. It is not hard to show that these matrices indeed satisfy the commutation relations (3.26). This completes our demonstration that the entire Lagrangian has a global $O(10-D, 10-D+N)$ symmetry.

\section{$3.4 D=4$ coset}

In four dimensions there is an additional axion, over and above those of the generic $D$ dimensional discussion, which arises if the 2-form potential $A_{(2)}$ is dualised. If $A_{(2)}$ is left undualised, the scalar Lagrangian will have an $O(6, N+6) \times \mathbb{R}$ global symmetry, as one would expect from the general results of the previous subsection. If $A_{(2)}$ is dualised, the symmetry group enlarges to $O(6,6+N) \times S L(2, \mathbb{R})$. We can see this very easily in the formalism that we have been using in this paper.

To include the effect of dualising $A_{(2)}$ to give an additional axion, we first add the kinetic term for $A_{(2)}$ to the scalar Lagrangian. Together with the kinetic term for $\phi$, this extra term gives

$$
e^{-1} \mathcal{L}_{\text {extra }}=-\frac{1}{2}(\partial \phi)^{2}-\frac{1}{2} e^{-2 \phi} F_{(3)}^{2},
$$

where $\phi=-\frac{1}{2} \vec{a}_{123} \cdot \vec{\phi}$. This is the linear combination of the dilatons which, as discussed in the previous subsection, is decoupled from the rest of the scalar Lagrangian. In the absence of the extra term (3.32), it would be responsible for contributing the extra $\mathbb{R}$ factor in the global symmetry. If we now dualise $A_{(2)}$, the term (3.32) gives the additional contribution

$$
e^{-1} \mathcal{L}_{(\phi, \chi)}=-\frac{1}{2}(\partial \phi)^{2}-\frac{1}{2} e^{2 \phi}(\partial \chi)^{2}
$$

\footnotetext{
${ }^{3}$ It is necessary, once again, to translate between the $2 \leq \alpha \leq 11-D$ notation and the $1 \leq i \leq 10-D$ notation.
} 
to the scalar Lagrangian, where

$$
F_{(3)}=e^{-2 \phi} * d \chi
$$

and $\chi$ is the new axion dual to $A_{(2)}$. Since the dilaton/axion system $(\phi, \chi)$ is decoupled from the rest of the scalar Lagrangian, it follows that the total global symmetry group for the scalar sector is now the direct product $O(6,6+N) \times S L(2, \mathbb{R})$.

This global symmetry extends to the full four-dimensional theory. To see this, we note that since the Bianchi identity for $F_{(3)}$ gives $d F_{(3)}=\frac{1}{2} d B_{(1)}^{I} \wedge d B_{(1)}^{I}-d A_{(1) \alpha} \wedge d \mathcal{A}_{(1)}^{\alpha}$, the dualisation of $A_{(2)}$ to $\chi$ will also give the extra contribution $\frac{1}{2} \chi *\left(d B_{(1)}^{I} \wedge d B_{(1)}^{I}-2 d A_{(1) \alpha} \wedge\right.$ $\left.d \mathcal{A}_{(1)}^{\alpha}\right)$ to the dualised Lagrangian. In the notation of section 2.3, the full Lagrangian can therefore be written as

$$
\begin{aligned}
e^{-1} \mathcal{L}_{4}= & R-\frac{1}{2}(\partial \phi)^{2}-\frac{1}{2} e^{2 \phi}(\partial \chi)^{2}+\frac{1}{4} \operatorname{tr}\left(\partial \mathcal{M}^{-1} \partial \mathcal{M}\right), \\
& -\frac{1}{4} e^{-\phi} H_{(2)}^{\mathrm{T}} \mathcal{M} H_{(2)}+\frac{1}{2} \chi *\left(H_{(2)}^{\mathrm{T}} \Omega H_{(2)}\right),
\end{aligned}
$$

where $\mathcal{M}$ is a parameterisation of the coset $O(6, N+6) /(O(6) \times O(N+6))$. As well as the manifest global $O(6, N+6)$ symmetry of the Lagrangian, there is also an $S L(2, \mathbb{R})$ symmetry of the equations of motion, under which $\mathcal{V} H_{(2)}$ and $e^{-\phi} *\left(\mathcal{V} H_{(2)}\right)^{\mathrm{T}}$ form an $S L(2, \mathbb{R})$ doublet.

\section{5 $D=3$ coset}

In three dimensions, the discussion of subsection 3.3 shows that if one leaves the higherdegree fields in their undualised form, the global symmetry group will be $O(7,7+N) \times \mathbb{R}$. If one dualises the vector potentials $\left(\mathcal{A}_{(1)}^{\alpha}, A_{(1) \alpha}, B_{(1)}^{I}\right)$ to give an additional $7+7+N$ axions $\left(\tilde{\chi}_{\alpha}, \chi^{\alpha}, \lambda_{I}\right)$, then the global symmetry group enlarges to $O(8,8+N)$. Note that the entire bosonic sector is now composed only of scalar fields.

To see how the symmetry enlarges, we first perform the dualisations specified above. To do this, we begin by obtaining the Bianchi identities for the 2 -form field strengths. From the results in Appendix A, we find the following:

$$
\begin{aligned}
d \mathcal{F}_{(2)}^{\alpha} & =\mathcal{F}_{(1) \beta}^{\alpha} \wedge \mathcal{F}_{(2)}^{\beta} \\
d F_{(2) \alpha} & =-\mathcal{F}_{(1) \alpha}^{\beta} \wedge F_{(2) \beta}-F_{(1) \alpha \beta} \wedge \mathcal{F}_{(2)}^{\beta}+G_{(1) \alpha}^{I} \wedge G_{(2)}^{I} \\
d G_{(2)}^{I} & =G_{(1) \alpha}^{I} \wedge \mathcal{F}_{(2)}^{\alpha} .
\end{aligned}
$$

Adding the appropriate Lagrange multipliers to the original Lagrangian, namely the terms

$$
\begin{aligned}
\mathcal{L}_{\mathrm{LM}}= & \tilde{\chi}_{\alpha}\left(d \mathcal{F}_{(2)}^{\alpha}-\mathcal{F}_{(1) \beta}^{\alpha} \wedge \mathcal{F}_{(2)}^{\beta}\right)+\lambda_{I}\left(d G_{(2)}^{I}-G_{(1) \alpha}^{I} \wedge \mathcal{F}_{(2)}^{i} \alpha\right) \\
& -\chi^{\alpha}\left(d F_{(2) \alpha}+\mathcal{F}_{(1) \alpha}^{\beta} \wedge F_{(2) \beta}+F_{(1) \alpha \beta} \wedge \mathcal{F}_{(2)}^{\beta}-G_{(1) \alpha}^{I} \wedge G_{(2)}^{I}\right),
\end{aligned}
$$


we find, after eliminating the 2-form field strengths by solving algebraically for them, that the dualised 1-form field strengths are given by

$$
\begin{aligned}
\mathcal{F}_{(1) \alpha} & \equiv-e^{\vec{b}_{\alpha} \cdot \vec{\phi}} * \mathcal{F}_{(2)}^{\alpha}=d \widetilde{\chi}_{\alpha}+\tilde{\chi}_{\beta} \mathcal{F}_{(1) \alpha}^{\beta}-\chi^{\beta} F_{(1) \alpha \beta}+\lambda_{I} G_{(1) \alpha}^{I}, \\
F_{(1)}^{\alpha} & \equiv e^{\vec{a}_{1 \alpha} \cdot \vec{\phi}} * F_{(2) \alpha}=d \chi^{\alpha}-\chi^{\beta} \mathcal{F}_{(1) \beta}^{\alpha} \\
G_{(1) I} & \equiv-e^{\vec{c} \cdot \vec{\phi}} * G_{(2)}^{I}=d \lambda_{I}-\chi^{\alpha} G_{(1) \alpha}^{I}
\end{aligned}
$$

(The sign differences in the Lagrange multiplier and definition of the field strength for the $\chi^{\alpha}$ terms are purely conventional, and have been introduced in order to simplify the form of the final result.)

Looking at the dilaton vectors for the full set of 1-form field strengths, we find that they are as follows:

$$
\begin{array}{rlll}
\mathcal{F}_{(1) \beta}^{\alpha}: & \vec{b}_{\alpha \beta}=-\vec{c}_{\alpha}+\vec{c}_{\beta}, & F_{(1)}^{\alpha}: & -\vec{a}_{1 \alpha}=-\vec{c}_{\alpha}+\vec{c}_{9}, \\
F_{(1) \alpha \beta}: & \vec{a}_{1 \alpha \beta}=\vec{c}_{\alpha}+\vec{c}_{\beta}, \quad & \mathcal{F}_{(1) \alpha}: & -\vec{b}_{\alpha}=\vec{c}_{\alpha}+\vec{c}_{9}, \\
G_{(1) \alpha}^{I}: & \vec{c}_{\alpha}, & G_{(1) I}: & -\vec{c}=\vec{c}_{9},
\end{array}
$$

where

$$
\vec{c}_{A}=\left(\vec{c}_{\alpha}, \vec{c}_{9}\right), \quad \vec{c}_{A} \cdot \vec{c}_{B}=2 \delta_{A B}
$$

We see that by writing the dilaton vector $-\vec{c}$ for the axions coming from the dualisation of $G_{(2)}^{I}$ as $\overrightarrow{c_{9}}$, it is then natural to extend the index range from $\vec{c}_{\alpha}$ with $2 \leq \alpha \leq 8$ to $\vec{c}_{A}$ with $2 \leq A \leq 9$. The sets of field strengths on each line in (3.39) then naturally pair together to make an extended set.

Let us define new extended sets of potentials $\bar{A}_{(0) A A}, \overline{\mathcal{A}}_{(0) B}^{A}$ and $\bar{B}_{(0) A}^{I}$ by

$$
\begin{aligned}
& \bar{A}_{(0) \alpha \beta}=A_{(0) \alpha \beta}, \quad \bar{A}_{(0) \alpha 9}=\tilde{\gamma}_{\alpha}^{\beta} \tilde{\chi}_{\beta}+\frac{1}{2} B_{(0) \alpha}^{I} \lambda_{I}, \\
& \overline{\mathcal{A}}_{(0) \beta}^{\alpha}=\mathcal{A}_{(0) \beta}^{\alpha}, \quad \overline{\mathcal{A}}_{(0) 9}^{\alpha}=\chi^{\alpha}, \\
& \bar{B}_{(0) \alpha}^{I}=B_{(0) \alpha}^{I}, \quad \bar{B}_{(0) 9}^{I}=\lambda_{I} .
\end{aligned}
$$

We also can define an extended set of matrices $\bar{\gamma}_{B}^{A}$, and their inverses $\widetilde{\bar{\gamma}}_{B}^{A} \equiv \delta_{B}^{A}+\overline{\mathcal{A}}_{(0) B}^{A}$. From the definitions (3.41), it follows that

$$
\bar{\gamma}_{\beta}^{\alpha}=\gamma_{\beta}^{\alpha}, \quad \bar{\gamma}_{9}^{\alpha}=\gamma_{\beta}^{\alpha} \chi^{\beta}, \quad \bar{\gamma}_{9}^{9}=1 .
$$

(As usual, $\bar{\gamma}_{B}^{A}$ is zero if $A>B$.) From the above, we then find that the extended set of 1-form field strengths $\bar{F}_{(1) A A}, \overline{\mathcal{F}}_{(1) B}^{A}$ and $\bar{G}_{(1) A}^{I}$ can be written as

$$
\begin{aligned}
\bar{F}_{(1) A B} & =\bar{\gamma}_{A}^{C} \bar{\gamma}_{B}^{D}\left(d \bar{A}_{(0) C D}-\bar{B}_{(0)[C}^{I} d \bar{B}_{(0) D]}^{I}\right), \\
\overline{\mathcal{F}}_{(1) B}^{A} & =\bar{\gamma}_{B}^{C} d \overline{\mathcal{A}}_{(0) C}^{A}, \quad \bar{G}_{(1) A}^{I}=\bar{\gamma}_{A}^{B} d \bar{B}_{(0) B}^{I} .
\end{aligned}
$$


In terms of these extended sets of fields, the fully-dualised three-dimensional Lagrangian is given by

$$
e^{-1} \mathcal{L}_{3}=-\frac{1}{2}(\partial \vec{\phi})^{2}-\frac{1}{2} \sum_{A<B} e^{\vec{b}_{A B} \cdot \vec{\phi}}\left(\overline{\mathcal{F}}_{(0) B}^{A}\right)^{2}-\frac{1}{2} \sum_{A<B} e^{\vec{a}_{A B} \cdot \vec{\phi}}\left(\bar{F}_{(1) A B}\right)^{2}-\frac{1}{2} \sum_{A, I} e^{\vec{c}_{A} \cdot \vec{\phi}}\left(\bar{G}_{(1) A}^{I}\right)^{2},
$$

where $\vec{b}_{A B}=-\vec{c}_{A}+\vec{c}_{B}, \vec{a}_{A B}=\vec{c}_{A}+\vec{c}_{B}$ and $\vec{c}_{A} \cdot \vec{c}_{B}=2 \delta_{A B}$. Thus we see that the $D=3$ Lagrangian has the identical form as (3.20), except that the index range of $A$ is extended to $2 \leq A \leq 9$, rather than the $1 \leq i \leq 10-D$ range occurring in (3.20). It follows from the discussion in subsection 3.3 that the Lagrangian (3.44) is therefore described by the coset $O(8,8+N) /(O(8) \times O(8+N))$.

\section{Time-like reductions of the heterotic string theory}

Dimensional reductions on Lorentzian tori have been discussed in [25, 26, 27, 28, 29, 30]. It was shown that the global symmetry groups remain unchanged from those of the usual Euclidean-torus reductions, but the coset structure is changed by virtue of the fact that the previous compact denominator groups are replaced by certain non-compact versions of these groups.

In this section, we give explicit derivations of the coset structure in all dimensions $3 \leq D \leq 9$, using the techniques that we have presented earlier in this paper. As we showed in section 2.2, the denominator groups $K$ for cosets $G / K$, where $G=O(p, q)$, are determined by the choice of a fiducial matrix $W_{0}$ lying on a particular orbit of the matrices $W$ satisfying $(2.13,2.14)$. In the discussion in section 2.3 , we saw that the matrices $\mathcal{M}$ that parameterise the scalar manifolds in dimensionally reduced Lagrangians played the rôle of the matrices $W$, and that the fiducial matrix $W_{0}$ could be read off simply by setting all the scalar fields to zero. In the usual reductions on Euclidean tori, the fiducial matrix is always just the identity, and hence it follows that the denominator group is the compact form $O(p) \times O(q)$.

For reductions on Lorentzian tori, a general discussion can easily be given for the cases $D \geq 5$. As in section 2.3, the $\mathcal{M}$ matrix can be read off from the kinetic terms of the 2 -form field strengths. The fiducial matrix where all the scalars vanish is diagonal, with unit-magnitude components whose signs are determined by the signs of the kinetic terms for the corresponding 2-form field strengths. Without loss of generality,flet us consider the case where the time-like reduction step is the one from $D=10$ to $D=9$, corresponding, in

\footnotetext{
${ }^{4}$ It was shown in 29 that time-like and space-like reduction steps commute.
} 
our notation, to the internal index $\alpha$ taking the value 2 . It follows that all lower-dimensional fields that have a single $\alpha=2$ internal index suffer a sign-reversal for their kinetic terms 29]. This means that the two 2 -form field strengths $\mathcal{F}_{(2)}^{\alpha}$ and $F_{(2) \alpha}$ with $\alpha=2$ will acquire signreversed kinetic terms, while all the other 2 -forms will retain their standard signs. This pair of 2-forms can be seen to be associated with one symmetric pair of off-diagonal -1 entries in the metric $\Omega$ given in (2.26), and thus they are associated with one eigenvalue +1 and one eigenvalue -1 in the $O(10-D, 10-D+N)$-invariant metric $\Omega$. This means that in terms of the diagonal invariant metric that we used in section 2.2, the fiducial matrix $W_{0}$ is given by (2.16) with $m=n=1$. Thus the coset spaces describing scalar manifolds in the $D$-dimensional theories obtained by time-like reductions to $D \geq 5$ are

$$
\frac{O(10-D, 10-D+N)}{O(1,9-D) \times O(1,9-D+N)} \times \mathbb{R} .
$$

There is an alternative way to determine the fiducial matrix $W_{0}$, which will prove to be useful later when we look at the time-like reduction down to $D=3$. From the form of the Kaluza-Klein metric reduction ansatz (A.2), we see that the effect of making a time-like reduction in the step from $D=10$ to $D=9$ can be achieved by performing the complex field redefinition

$$
\vec{\phi} \longrightarrow \vec{\phi}+\frac{\mathrm{i} \pi}{2}\left(\vec{c}_{2}-\vec{c}\right)
$$

(Note that the ten-dimensional dilaton $\phi_{1}$ is unchanged under this redefinition.) From (2.24), we see that upon setting all the axions to zero, the matrix $\mathcal{M}=\mathcal{V}^{\mathrm{T}} \mathcal{V}$ is given by

$$
\mathcal{M}=\operatorname{diag}\left(e^{\vec{c}_{\alpha} \cdot \vec{\phi}} \delta_{\alpha}^{\beta}, \delta_{I}^{J}, e^{-\vec{c}_{\alpha} \cdot \vec{\phi}} \delta_{\beta}^{\alpha}\right)
$$

for the usual case of reduction on a Euclidean torus. The transformation (4.2) then implies that there is a sign reversal on the two components corresponding to $\alpha=2$. If we now set the dilatons to zero, we get precisely the same fiducial matrix as described above.

In $D=4$, the global symmetry group is $O(6,6+N) \times S L(2, \mathbb{R})$. The same considerations as given above show that the stability subgroup of the $O(6,6+N)$ factor is $O(1,5) \times O(1,5+$ $N)$. The stability subgroup of the $S L(2, \mathbb{R})$ factor, which would be $O(2)$ in a standard Euclidean-torus reduction, will now instead be $O(1,1)$. The reason for this is that the kinetic term for the axion $\chi$ in (3.33) will be reversed when the dualisation from (3.32) is performed in the four-dimensional Euclidean-signatured space. Thus the coset for the scalar manifold in $D=4$ will be

$$
\frac{O(6, N+6)}{O(1,5) \times O(1, N+5)} \times \frac{S L(2, \mathbb{R})}{O(1,1)} .
$$


In $D=3$, the global symmetry group is $O(8, N+8)$. In this case, upon setting all the axions to zero, the $(N+16) \times(N+16)$ matrix $\mathcal{M}$ is given by

$$
\mathcal{M}=\operatorname{diag}\left(e^{\vec{c}_{A} \cdot \vec{\phi}} \delta_{A}^{B}, \delta_{I}^{J}, e^{-\vec{c}_{A} \cdot \vec{\phi}} \delta_{B}^{A}\right),
$$

where $2 \leq A \leq 9$ (see section 3.5). The field redefinition (4.2) implies that there will now be four terms in (4.5) that undergo sign reversals, namely those corresponding to $A=2$ and $A=9$. Thus, setting the dilatons to zero, we obtain a fiducial matrix of the form (2.16) with $m=n=2$. Consequently, the coset space describing the theory in $D=3$ obtained by timelike reduction has the form

$$
\frac{O(8, N+8)}{O(2,6) \times O(2, N+6)} .
$$

The coset structures for Lorentzian torus reductions that we have derived in this section are all in agreement with those given in 28.

\section{$5 \quad$ K3 compactifications of M-theory}

Now let us consider, by contrast, the K3 reduction of $D=11$ supergravity. We aim to show in some detail the relations between this theory and the $T^{3}$ reduction of the $E_{8} \times E_{8}$ heterotic theory as discussed above, which are conjectured to be equivalent under duality [1, 2]. The numbers of zero modes in the two theories may be compared straightforwardly by counting the harmonic forms corresponding to the various $D=7$ spin sectors in each theory.

Reducing $D=11$ supergravity on K3, one obtains a $D=7$ metric and 58 scalars from the reduction of the $D=11$ metric, plus a $D=7$ three-form antisymmetric tensor gauge potential plus 22 one-form gauge potentials from the reduction of the $D=11$ three-form gauge potential. The 58 scalars arise from the 57 shape-determining plus one volume-setting moduli of the internal K3 manifold, while the 22 one-forms arise from the 22 harmonic twoforms occurring on K3.

In the supergravity sector of the heterotic theory compactified on $T^{3}$, one has for com-

parison: a $D=7$ metric plus a triplet of dilatonic scalars, together with a triplet of one-form Kaluza-Klein gauge potentials and a triplet of axionic scalars, all descending from the $D=10$ metric; one additional dilatonic scalar descending from the $D=10$ dilaton; and also a $D=7$ two-form gauge potential, three one-form gauge potentials and three axionic scalars, all descending from the $D=10$ two-form gauge potential. The Yang-Mills sector of the heterotic theory contributes a number of $D=7$ zero-modes as well. We shall assume for this accounting that the Yang-Mills gauge symmetry is "fully Higgsed," i.e. that the gauge 
symmetry is maximally broken by giving vacuum expectation values to the various $E_{8} \times E_{8}$ adjoint representation scalar fields. This breaks the $E_{8} \times E_{8}$ group down to its Cartan subgroup, $(U(1))^{16}$. Thus we get 16 further vector potentials and $16 \times 3=48$ axionic scalars from the Yang-Mills sector. Comparing with the K3 reduction of M-theory as described above, we see that we have a total of 22 vector potentials in each case, and 58 scalars in each case. From the K3 reduction we have a 3 -form potential in $D=7$, while from the $T^{3}$ reduction of the heterotic theory we have a 2 -form potential instead. This indicates that one needs to perform a dualisation of one of the two seven-dimensional theories in order to make contact with the other. In particular, this indicates that the relation between the two involves an interchange between strong and weak coupling regimes.

To make things more precise, let us begin by looking in detail at the $T^{3}$ reduction of the heterotic theory. In order to make contact with the K3 reduction of $D=11$ supergravity, we will make a dualisation of the 2 -form potential $A_{(2)}$ arising in the $T^{3}$ reduction of the heterotic theory. To do this, we need to know the Bianchi identity for the field strength $F_{(3)}$. From the results given in Appendix A, we find this to be $d F_{(3)}+F_{(2) \alpha} \wedge \mathcal{F}_{(2)}^{\alpha}+\frac{1}{2} G_{(2)}^{I} \wedge G_{(2)}^{I}=0$. To dualise $A_{(2)}$, we introduce a 3 -form $A_{(3)}$ as a Lagrange multiplier, adding the term $A_{(3)} \wedge\left(d F_{(3)}+F_{(2) \alpha} \wedge \mathcal{F}_{(2)}^{\alpha}+\frac{1}{2} G_{(2)}^{I} \wedge G_{(2)}^{I}\right)$ to the undualised Lagrangian. Treating $F_{(3)}$ now as an auxiliary field, we can solve its algebraic equation of motion, giving $e^{\vec{a}_{1} \cdot \vec{\phi}} * F_{(3)}=d A_{(3)} \equiv F_{(4)}$. Substituting this back into the Lagrangian, we obtain the dualised version

$$
\begin{aligned}
e^{-1} \mathcal{L}_{7}= & R-\frac{1}{2}(\partial \vec{\phi})^{2}-\frac{1}{48} e^{-\vec{a}_{1} \cdot \vec{\phi}}\left(F_{(4)}\right)^{2}-\frac{1}{4} \sum_{\alpha} e^{\vec{a}_{1 \alpha} \cdot \vec{\phi}}\left(F_{(2) \alpha}\right)^{2} \\
& -\frac{1}{2} \sum_{\alpha<\beta} e^{\vec{a}_{1 \alpha \beta} \cdot \vec{\phi}}\left(F_{(1) \alpha \beta}\right)^{2}-\frac{1}{2} \sum_{I} e^{\vec{c} \cdot \vec{\phi}}\left(G_{(2)}^{I}\right)^{2}-\frac{1}{2} \sum_{\alpha, I} e^{\vec{c}_{\alpha} \cdot \vec{\phi}}\left(G_{(1) \alpha}^{I}\right)^{2} \\
& -\frac{1}{4} \sum_{\alpha} e^{\vec{b}_{\alpha} \cdot \vec{\phi}}\left(\mathcal{F}_{(2)}^{\alpha}\right)^{2}-\frac{1}{2} \sum_{\alpha<\beta} e^{\vec{b}_{\alpha \beta} \cdot \vec{\phi}}\left(\mathcal{F}_{(1) \beta}^{\alpha}\right)^{2} \\
& +*\left(A_{(3)} \wedge\left(d A_{(1) \alpha}^{\prime} \wedge d \hat{\mathcal{A}}_{(1)}^{\alpha}+\frac{1}{2} d B^{I^{\prime}}{ }_{(1)} \wedge d B^{I^{\prime}}{ }_{(1)}\right)\right)
\end{aligned}
$$

where $F_{(4)}=d A_{(3)}$, and the other field strengths are given in (A.10).

To make comparison with the K3 reduction of $D=11$ supergravity, we first need to discuss the nature of the K3 manifold. (A detailed account of its properties may be found in [31.) The required K3 metric is Ricci flat and Kähler. Although an existence proof for Ricci-flat metrics on $\mathrm{K} 3$ has been given long ago [32], the explicit form of such metrics is still unknown, owing to the complexity of the Einstein equation. It is, however, possible to give an approximate construction of the Ricci-flat metrics (see, for example, [33, 13]). A detailed discussion of this "physical" picture was given in [13]. One can construct K3 by beginning with the 4 -torus $T^{4}$, defined by identifying coordinates $y^{i} \sim y^{i}+2 \pi$ in $\mathbb{R}^{4}$. Next, 
we make the identification $y^{i} \sim-y^{i}$. This identification has 16 fixed points, located at $y^{i}=\pi n_{i}$, where $n_{i}$ are any integers. One then cuts out a small 4 -ball around each of the 16 fixed points. Had we not performed the identifications, the boundaries of the 4-balls would each have been a 3-sphere. Because of the identification, the boundaries are instead copies of $R P^{3}$, the real projective plane. (This is $S^{3}$ with an antipodal identification.) One now patches up the manifold, by "plugging in" an appropriate space into each of the 4-balls. What is needed is a smooth 4-space with curvature localised to a small region, and which then opens out into an asymptotic region that approaches flat Euclidean 4-space but with an antipodal identification so that its boundary is again $R P^{3}$. Such a space is known; it is the Eguchi-Hanson instanton [34], which indeed approaches Euclidean space factored by $Z_{2}$ at infinity [35]. By taking the "size" of the instanton to be sufficiently small, one achieves an almost-smooth join between the torus and the Eguchi-Hanson instanton, which splays out like a champagne cork and plugs into the excised 4-ball in the 4-torus. Inserting a total of 16 such corks, i.e. one for each excised 4-ball, one achieves an approximation to a Ricciflat K3 manifold that becomes arbitrarily precise as the scale sizes of the Eguchi-Hanson instantons are taken to zero [13].

Using the above construction, it is possible to give a fairly explicit construction of the K3 compactification of $D=11$ supergravity. In particular the harmonic 2-forms on K3, in terms of which all of the non-trivial zero-modes are described, can be seen to fall into two different categories. First of all, there are those that can be viewed as being harmonic 2 -forms on the 4 -torus. In fact, one can easily see that the set of harmonic forms on the 4-torus that will be present in the K3 itself will be the subset that survives the antipodal identification $y^{i} \sim-y^{i}$. Thus the six harmonic 2 -forms $d y^{i} \wedge d y^{j}$ survive, whilst the four harmonic 1-forms $d y^{i}$ are projected out. Thus we may define three self-dual 2-forms $J_{+}^{\alpha}$ and three anti-self-dual 2-forms $J_{-}^{\alpha}$ on $T^{4}$ (with the triplet index $\alpha$ running over the values $2,3,4$ in order to match with the notation arising in the $T^{3}$ compactification of the heterotic string):

$$
\begin{aligned}
J_{ \pm}^{2} & =d y^{1} \wedge d y^{4} \pm d y^{2} \wedge d y^{3} \\
J_{ \pm}^{3} & =d y^{2} \wedge d y^{4} \pm d y^{3} \wedge d y^{1} \\
J_{ \pm}^{4} & =d y^{3} \wedge d y^{4} \pm d y^{1} \wedge d y^{2}
\end{aligned}
$$

The second type of harmonic 2-forms are those associated with the Eguchi-Hanson instantons. There is one of these for each of the sixteen instantons, corresponding to the fact that the Eguchi-Hanson solution has one normalisable harmonic 2-form. We shall 
represent these 2 -forms by the symbols $\omega_{(2)}^{I}$. Each of these 2-forms is strongly localised within a small region of the Eguchi-Hanson space itself. Concretely, the Eguchi-Hanson metric is given by [34]

$$
d s^{2}=\left(1-\frac{a^{4}}{r^{4}}\right)^{-1} d r^{2}+\frac{1}{4} r^{2}\left(1-\frac{a^{4}}{r^{4}}\right)(d \psi+\cos \theta d \varphi)^{2}+\frac{1}{4} r^{2}\left(d \theta^{2}+\sin ^{2} \theta d \varphi^{2}\right) .
$$

The radial coordinate has the range $a \leq r<\infty$, with the bolt occurring at $r=a$. The coordinates $\theta$ and $\varphi$ are angles on $S^{2}$. The level surfaces $r=$ constant have the topology of $R P^{3}$, since the fibre coordinate $\psi$ has period $2 \pi$ rather than the $4 \pi$ period that would occur on $S^{3}$ 35. In the natural orthonormal frame $e^{0}=\left(1-a^{4} / r^{4}\right)^{-1 / 2} d t, e^{1}=\frac{1}{2} r d \theta$, $e^{2}=\frac{1}{2} r \sin \theta d \varphi, e^{3}=\frac{1}{2} r\left(1-a^{4} / r^{4}\right)^{1 / 2}(d \psi+\cos \theta d \varphi)$, it is easy to see that the anti-self-dual 2 -form

$$
\omega_{(2)}=\frac{1}{r^{4}}\left(e^{0} \wedge e^{3}-e^{1} \wedge e^{2}\right)
$$

is closed. This is the normalisable anti-self-dual harmonic 2-form on Eguchi-Hanson.

In total, we then have 22 harmonic 2-forms; six from $T^{4}$ plus 16 from the corks. These divide into 3 self-dual harmonic 2 -forms from $T^{4}$, plus $19=3+16$ anti-self-dual harmonic 2-forms, coming from $T^{4}$ and from the 16 Eguchi-Hanson metrics. As we shall see, the ways in which the 2-forms from $T^{4}$ and from the Eguchi-Hansons contribute in the dimensional reduction procedure will be slightly different.

Upon performing the $\mathrm{K} 3$ reduction, the $D=11$ fields give rise to the following $D=7$ fields:

$$
\begin{aligned}
& g_{M N} \longrightarrow g_{\mu \nu}, \quad \mathcal{A}_{(0) j}^{i}, \quad \mathcal{A}_{(0) \alpha}^{I}, \quad \vec{\phi} \\
& A_{(3)} \longrightarrow A_{(3)}, \quad A_{(1) i j}, \quad A_{(1) I} \text {. }
\end{aligned}
$$

Here, as usual, the $i, j, \ldots$ indices range over the four internal coordinates $y^{i}$. There are four dilatons $\vec{\phi}$, arising from the fact that in the construction of K3 that we are using here, there are the usual four circles making up the 4-torus. There are also six axions $\mathcal{A}_{(0) j}^{i}(i<j)$ that parameterise the angular deformations of the 4-torus. There are also $48=16 \times 3$ further scalars $\mathcal{A}_{(0) \alpha}^{I}$, corresponding to the remaining parameters that make up the total of $58=10+48$ moduli of K3 [36, 37]. These can be understood in the picture of K3 that we are using as follows. There are two parameters that characterise the orientation of each of the Eguchi-Hanson instantons, and a further parameter characterising its scale size. This gives $16 \times(2+1)=48$ parameters in total that are associated with the Eguchi-Hanson corks. The 22 vector fields that we mentioned previously split as six vectors $A_{(1) i j}$ coming 
from the $T^{4}$ reduction of $A_{3}$, plus 16 vectors $A_{(1) I}$ coming from the harmonic expansion involving the 16 harmonic 2-forms $\omega_{(2)}^{I}$ localised in the 16 Eguchi-Hanson instantons.

The dilaton couplings for each field can be obtained straightforwardly, by examining the ansatz for the reduction of the eleven-dimensional metric on $T^{4}$ :

$$
d s_{11}^{2}=e^{\frac{1}{3} \vec{g} \cdot \vec{\phi}} d s_{7}^{2}+\sum_{i=1}^{4} e^{2 \vec{\gamma}_{i} \cdot \vec{\phi}}\left(d z^{i}+\mathcal{A}_{(0) j}^{i} d z^{j}\right)^{2} .
$$

The constant vectors $\vec{g}$ and $\vec{\gamma}_{i}$ can be found in [5, 39], and are given by

$$
\begin{aligned}
& \vec{\gamma}_{1}=\left(-\frac{2}{3}, 0,0,0\right), \quad \vec{\gamma}_{2}=\left(\frac{1}{12},-\frac{\sqrt{7}}{4}, 0,0\right), \\
& \vec{\gamma}_{3}=\left(\frac{1}{12}, \frac{1}{4 \sqrt{7}},-\sqrt{\frac{3}{7}}, 0\right), \quad \vec{\gamma}_{4}=\left(\frac{1}{12}, \frac{1}{4 \sqrt{7}}, \frac{1}{2 \sqrt{21}},-\sqrt{\frac{5}{12}}\right), \\
& \vec{g}=-\frac{6}{5} \sum_{i} \vec{\gamma}_{i}=\left(\frac{1}{2}, \frac{3}{2 \sqrt{7}}, \sqrt{\frac{3}{7}}, \sqrt{\frac{3}{5}}\right) .
\end{aligned}
$$

Note that the radius $R_{i}$ of the $i$ 'th circle on $T^{4}$, and the $T^{4}$ volume $V_{4}=\prod_{i} R_{i}$, are given by

$$
R_{i}=e^{\vec{\gamma}_{i} \cdot \vec{\phi}}, \quad V_{4}=e^{-\frac{5}{6} \vec{g} \cdot \vec{\phi}}
$$

Thus the combination of dilatons $\varphi=\sqrt{5 / 8} \vec{g} \cdot \vec{\phi}$ is the breathing mode of $T^{4}$, and hence also of K3. It is also sometimes useful to present another form of the metric reduction ansatz that is applicable to computations that do not involve the "internal" structure of the compactifying 4-manifold, but only depend on the breathing mode. This is given by

$$
d s_{11}^{2}=V_{4}^{-2 / 5} d s_{7}^{2}+V_{4}^{1 / 2} d s_{4}^{2}
$$

Associating dilaton vectors with the various seven-dimensional fields as follows,

$$
\begin{array}{ccccc}
\mathcal{A}_{(0) j}^{i} & \mathcal{A}_{(0) \alpha}^{I} & A_{(3)} & A_{(1) i j} & A_{(1) I} \\
\vec{b}_{i j} & \vec{b}_{\alpha} & \vec{a} & \vec{a}_{i j} & \vec{d}
\end{array},
$$

we find that they can be expressed in terms of $\vec{g}$ and $\vec{\gamma}_{i}$ as

$$
\begin{aligned}
& \vec{b}_{i j}=2 \vec{\gamma}_{i}-2 \vec{\gamma}_{j}, \quad \vec{b}_{2}=\vec{\gamma}_{1}+\vec{\gamma}_{2}-\vec{\gamma}_{3}-\vec{\gamma}_{4}, \\
& \vec{b}_{3}=\vec{\gamma}_{2}+\vec{\gamma}_{3}-\vec{\gamma}_{1}-\vec{\gamma}_{4}, \quad \vec{b}_{4}=\vec{\gamma}_{3}+\vec{\gamma}_{1}-\vec{\gamma}_{2}-\vec{\gamma}_{4}, \\
& \vec{a}=-\vec{g}, \quad \vec{a}_{i j}=-2 \vec{\gamma}_{i}-2 \vec{\gamma}_{j}-\frac{1}{3} \vec{g}, \quad \vec{d}=\frac{1}{2} \vec{g} .
\end{aligned}
$$

We shall now show in detail how these dilaton couplings arise in the K3 reduction of M-theory. The dilaton vectors $\vec{a}, \vec{a}_{i j}$ and $\vec{b}_{i j}$, corresponding to the fields $A_{(3)}, A_{(1) i j}$ and $\mathcal{A}_{(0) j}^{i}$ can be understood straightforwardly, since they are a subset of those one would obtain by dimensionally reducing M-theory on $T^{4}$. As we shall see, they are in fact nothing but 
an $S L(4, \mathbb{R})$ truncation of maximal supergravity in $D=7$, which has an $S L(5, \mathbb{R})$ global symmetry. To see this, consider the ansatz for the reduction of the 3 -form potential

$$
A_{(3)}(x, y)=A_{(3)}(x)+\frac{1}{2} A_{(1) i j}(x) \wedge d y^{i} \wedge d y^{j}+A_{(1) I}(x) \wedge \omega_{(2)}^{I} .
$$

For now, it is only the first two terms here that concern us. From the metric ansatz (5.6), we can see that the determinant of the vielbein reduces according to $e \rightarrow e e^{\frac{1}{3} \vec{g} \cdot \vec{\phi}}$, and thus we have that

$$
-\frac{1}{48} e F_{(4)}^{2} \longrightarrow-\frac{1}{48} e e^{-\vec{g} \cdot \vec{\phi}} F_{(4)}^{2}-\frac{1}{4} \sum_{i<j} e^{-\left(2 \vec{\gamma}_{i}+2 \vec{\gamma}_{j}+\frac{1}{3} \vec{g}\right) \cdot \vec{\phi}}\left(F_{(1) i j}\right)^{2}+\cdots
$$

where $\cdots$ represents the $F_{(2) I}$ terms that we shall discuss presently, and in obtaining the exponential factors we have used the appropriate inverse metric components in $D=7$ or $D=4$, as given in (5.6). The exponents can indeed be seen to be $\vec{a} \cdot \vec{\phi}$ and $\vec{a}_{i j} \cdot \phi$ in (5.11). The dilaton couplings for the axions $\mathcal{A}_{(0) j}^{i}$ coming from the torus reduction of the metric follow from the standard Kaluza-Klein formulae as given, for example, in [39]. In fact we can also obtain the result for these axions by a simple linearised calculation, and it is useful to present this here because a similar argument will be used below in discussing the more difficult case of the other 48 axions coming from the K3 metric moduli. If a metric $\bar{g}_{i j}$ is subjected to a transverse traceless perturbation $h_{i j}$, i.e. $g_{i j}=\bar{g}_{i j}+h_{i j}$ where $\bar{g}^{i j} h_{i j}=0$ and $\bar{\nabla}^{i} h_{i j}=0$, then the perturbed Ricci tensor will be of the form $R_{i j}=\bar{R}_{i j}+\frac{1}{2} \Delta_{L} h_{i j}$, where the Lichnerowicz operator $\Delta_{L}$ is defined by $\Delta_{L} h_{i j}=-\bar{\square} h_{i j}-$ $2 \bar{R}_{i k j \ell} h^{k \ell}+2 \bar{R}_{(i}{ }^{k} h_{j) k}$. The fluctuation $h_{i j}$ will therefore give rise to a contribution of the form $\frac{1}{2} h^{i j} \square h_{i j}$ in the Einstein-Hilbert Lagrangian, where $h^{i j}=\bar{g}^{i k} \bar{g}^{j \ell} h_{k \ell}$. In the present context, we see from (5.6) that the metric fluctuation corresponding to the axion $\mathcal{A}_{(0) j}^{i}$ is given by $h_{i j}=e^{2 \vec{\gamma}_{i} \cdot \vec{\phi}} \mathcal{A}_{(0) j}^{i}$ when $i<j$ (together with $h_{j i}=h_{i j}$ ). Thus we will get a contribution of the form $\frac{1}{2} e^{-2\left(\vec{\gamma}_{i}+\vec{\gamma}_{j}\right) \cdot \vec{\phi}} e^{2 \vec{\gamma}_{i} \cdot \vec{\phi}} \mathcal{A}_{(0) j}^{i} \square\left(e^{2 \vec{\gamma}_{i} \cdot \vec{\phi}} \mathcal{A}_{(0) j}^{i}\right) \sim-\frac{1}{2} e^{2\left(\vec{\gamma}_{i}-\vec{\gamma}_{j}\right) \cdot \vec{\phi}}\left(\partial \mathcal{A}_{(0) j}^{i}\right)^{2}$. This result shows that indeed the dilaton vector describing the dilaton coupling is the one given by $\vec{b}_{i j}$ in 5.11 .

It is a little more involved to understand the dilaton vectors $\vec{d}$ and $\vec{b}_{\alpha}$ describing the couplings of the 16 vector potentials $A_{(1) I}$ and the 48 axions $\mathcal{A}_{(0) \alpha}^{I}$, since these arise from the sixteen 2-form harmonics $\omega_{(2)}^{I}$ that are intrinsic to K3. Let us begin by considering the vector fields $A_{(1) I}$, which are the easier of the two sets to analyse. From the last term in (5.12), we see that these give the $d A_{(1) I} \wedge \omega_{(2)}^{I}$ contributions to the elevendimensional 4-form $F_{(4)}$. In the same spirit as above, we can calculate the dimensional reductions of these terms by making the necessary contractions of indices using the appropriate metric components as given by the Kaluza-Klein ansatz. For these fields, since their 
internal components involve $\omega_{(2)}^{I}$, we should use the metric given by (5.9). Thus we find $-\frac{1}{48} e F_{(4)}^{2} \rightarrow-\frac{1}{4} e V_{4}^{-2 / 5} V_{4}^{4 / 5} V_{4}^{-1}\left(F_{(2) I}\right)^{2}=-\frac{1}{4} e e^{\frac{1}{2} \vec{g} \cdot \vec{\phi}}\left(F_{(2) I}\right)^{2}$, which indeed agrees with the dilaton vector $\vec{d}$ given in (5.11).

The determination of the dilaton vectors for the $\mathcal{A}_{(0) \alpha}^{I}$ is more complicated. One approach is to note that the subgroup $G L(4, \mathbb{R}) \sim O(3,3) \times \mathbb{R}$ of the $O(3,19) \times \mathbb{R}$ global symmetry group of the K3 reduction corresponds precisely to the unbroken general coordinate symmetry on the $T^{4}$. The antipodal identification in the $T^{4}$ described above preserves this global symmetry group. All of the fields should therefore form representations under this $G L(4, \mathbb{R})$. This can be seen in particular in the couplings of the dilatonic scalars. Specifically, the dilaton vectors should form weight vectors under $G L(4, \mathbb{R})=\mathbb{R} \times S L(4, \mathbb{R})$. Here the $\mathbb{R}$ factor is generated by the breathing mode. Indeed, the dilaton couplings of $F_{(4)}$ and the $F_{(2)}^{I}$ depend only on the breathing mode, and they are accordingly singlets under $S L(4, \mathbb{R})$. On the other hand, the dilaton vectors $\vec{a}_{i j}$ of $F_{(2) i j}$ are precisely the weight vectors of the six-dimensional representation of $S L(4, \mathbb{R})$, after the subtraction of a universal constant vector associated with the breathing mode. The dilaton vectors $\vec{b}_{i j}$ for the scalars $\mathcal{A}^{i}{ }_{(0) j}$ form the positive roots of $S L(4, \mathbb{R})$, with simple roots $\vec{b}_{12}, \vec{b}_{23}$ and $\vec{b}_{34}$. To see that these axionic scalars, taken together with the dilatonic degrees of freedom orthogonal to the breathing mode, realise the full $S L(4, \mathbb{R})$, it is necessary to include the negatives of the dilaton vectors, i.e. $-\vec{b}_{i j}$, since the set of vectors $\pm \vec{b}_{i j}$ form the complete root system of $S L(4, \mathbb{R})$. Note, however, that dilatonic couplings with $-\vec{b}_{i j}$ do not occur in the Lagrangian. This is a reflection of the fact that the scalars parameterise the coset $S L(4, \mathbb{R}) / O(4)$, and thus provide a non-linear realisation of $S L(4, \mathbb{R})$. The negative roots are generated [38 by the non-linear (Weyl group) transformation $\mathcal{A}_{(0) j}^{i} \rightarrow e^{-\vec{b}_{i j} \cdot \vec{\phi}} \mathcal{A}_{(0) j}^{i}+\ldots$ Thus for the scalar sector, both the dilaton vectors and their negatives should be included in discussing the global symmetry.

In the approximate description of K3 that we are using here, we may note that each of the 16 fixed points under antipodal identification should be "patched" with an EguchiHanson instanton. The 16 instantons are equivalent, and so we can discuss just a single one of them as a representative. Each instanton contributes three metric zero modes, described by three axions. The insertion of the Eguchi-Hanson instantons preserves the $S L(4, \mathbb{R})$ symmetry of the original $T^{4}$, since, as we have observed, its asymptotic limit is the same as the antipodally-identified $T^{4}$. Following the above discussion, the three dilaton vectors and their negatives form a six-dimensional representation of $S L(4, \mathbb{R})$. (The inclusion of the negatives of the dilaton vectors is clearly necessary since there exists no triplet representation 
of $S L(4, \mathbb{R})$.) We now find that the set $\pm \vec{b}_{\alpha}$ form a six-dimensional representation of $S L(4, \mathbb{R})$, given the chosen basis $\vec{b}_{i j}$ for the positive roots. This representation is not unique, however; another example is the set $\vec{a}_{i j}$ discussed above. However, the set $\pm \vec{b}_{\alpha}$ given in (5.11) forms the unique solution in which three vectors together with their negatives comprise a six-dimensional representation. Thus we see that the forms of these dilaton vectors are dictated by the global symmetry $S L(4, \mathbb{R})$.

An alternative way to understand these dilaton vectors is to note that the K3 metric has 3 self-dual and 3+16 anti-self dual harmonic 2-forms. In the approximate K3 construction, we see that for each Eguchi-Hanson instanton there is one localised anti-self-dual harmonic 2 -form $\omega_{(2)}$, and three covariantly-constant self-dual 2-forms $J_{+}^{\alpha}$. As shown in [36], one can use these to build three zero-mode deformations of the metric (i.e. Lichnerowicz zeromodes) of the form $h_{i j}=J_{+i k}^{a} \omega^{k}$. This gives a total of $3 \times 16=48$ metric zero-modes, which, together with the 10 coming from the 4 -torus (4 dilatons $\vec{\phi}$ plus 6 axions $\mathcal{A}_{(0) j}^{i}$ ), give the 58 metric zero-modes of $\mathrm{K} 3$. In the bulk $T^{4} / Z_{2}$ part of $\mathrm{K} 3$, we have a total of six 1-form field strengths $\mathcal{F}_{(1) j}^{i}$ for six axions, forming three pairs $\mathcal{F}_{(1) \pm}^{2}=\mathcal{F}_{(1) 4}^{1} \pm \mathcal{F}_{(1) 3}^{2}$, $\mathcal{F}_{(1) \pm}^{3}=\mathcal{F}_{(1) 4}^{2} \pm \mathcal{F}_{(1) 1}^{3}=\mathcal{F}_{(1) 4}^{2} \mp \mathcal{F}_{(1) 3}^{1} e^{-\vec{b}_{13} \cdot \vec{\phi}}, \mathcal{F}_{(1) \pm}^{4}=\mathcal{F}_{(1) 4}^{3} \pm \mathcal{F}_{(1) 2}^{1}$. On the other hand, for each instanton there are just three axions. This difference is associated with the fact that, whereas in the $T^{4} / Z_{2}$ bulk there are 3 self-dual and 3 anti-self-dual constant harmonics associated with the above three pairs of axions, there are in each instanton just three selfdual covariantly constant 2 -forms. The nature of the dilatonic couplings for the three axions associated with each instanton can be revealed by first studying the detailed structure of the dilaton couplings for the bulk $T^{4} / Z_{2}$ pairs of field strengths $\mathcal{F}_{(1) \pm}^{\alpha}$. Let us consider the Lagrangian for the $\mathcal{F}_{(1) \pm}^{2}$ pair, which is given by

$$
\begin{aligned}
e^{-1} \mathcal{L} & =-\frac{1}{2}(\partial \vec{\phi})^{2}-\frac{1}{4} e^{\vec{b}_{14} \cdot \vec{\phi}}\left(\mathcal{F}_{(1) 4}^{1}\right)^{2}-\frac{1}{4} e^{\vec{b}_{23} \cdot \vec{\phi}}\left(\mathcal{F}_{(1) 3}^{2}\right)^{2} \\
& =-\frac{1}{2}(\partial \vec{\phi})^{2}-\frac{1}{4} e^{\frac{1}{2}\left(\vec{b}_{14}+\vec{b}_{23}\right) \cdot \vec{\phi}}\left(\mathcal{F}_{(1)+}^{2}, \mathcal{F}_{(1)-}^{2}\right)\left(\begin{array}{ll}
c & s \\
s & c
\end{array}\right)\left(\begin{array}{l}
\mathcal{F}_{(1)+}^{2} \\
\mathcal{F}_{(1)-}^{2}
\end{array}\right),
\end{aligned}
$$

where $c=\cosh \theta$ and $s=\sinh \theta$, with $\theta=\frac{1}{2}\left(\vec{b}_{14}-\vec{b}_{23}\right) \cdot \vec{\phi}$. Thus for $\mathcal{F}_{(1)+}^{2}$, the dilaton couplings are naturally described by $\frac{1}{2}\left(\vec{b}_{14}+\vec{b}_{23}\right)$. This is because we can set consistently set both $\mathcal{F}_{(1)-}^{2}=0$ and $\theta=0$, and then $\cosh \theta$ is replaced by unity in the dilaton coupling. Indeed, the natural augmentation of (5.14) to include additional axions $\psi_{I}$ gives

$$
e^{-1} \mathcal{L}=-\frac{1}{2}(\partial \vec{\phi})^{2}-\frac{1}{4} e^{\frac{1}{2}\left(\vec{b}_{14}+\vec{b}_{23}\right) \cdot \vec{\phi}}\left[\left(\mathcal{F}_{(1)+}^{2}, \mathcal{F}_{(1)-}^{2}\right)\left(\begin{array}{cc}
c & s \\
s & c
\end{array}\right)\left(\begin{array}{c}
\mathcal{F}_{(1)+}^{2} \\
\mathcal{F}_{(1)-}^{2}
\end{array}\right)+\sum_{I}\left(\partial \psi_{I}\right)^{2}\right] .
$$

\footnotetext{
${ }^{5}$ Note that the indices 1, 2 and 3 on the $\mathcal{F}_{(1)}$ fields are cyclic. In the Borel-type (Solvable Lie Algebra) gauge, the Lagrangian is expressed naturally in terms of $\mathcal{F}_{(1) j}^{i}$ with $i<j$ and we have $\mathcal{F}_{(1) j}^{i}=-\mathcal{F}_{(1) i}^{j} e^{\vec{b}_{j i} \cdot \vec{\phi}}$.
} 
In other words, the original pair of axions not only have an overall dilaton factor $e^{\frac{1}{2}\left(\vec{b}_{14}+\vec{b}_{23}\right) \cdot \vec{\phi}}$ but also the $\left(\begin{array}{ll}c & s \\ s & c\end{array}\right)$ matrix coupling, while all further axions $\psi_{i}$ are "unpaired," and have only the overall $e^{\frac{1}{2}\left(\vec{b}_{14}+\vec{b}_{23}\right) \cdot \vec{\phi}}$ factor. Thus we can argue that one of the three axions associated with a given Eguchi-Hanson instanton can naturally be grouped with the $\mathcal{F}_{(1) \pm}^{2}$, with a Lagrangian contribution of the same form as those of the $\psi_{I}$ in (5.15). This is because the three axions can be approximately viewed as an internal self-dual truncation (analogous to setting $\mathcal{F}_{(1)-}^{2}$ to zero), since there are only three self-dual constant harmonic 2 -forms in the Eguchi-hanson metric. This implies that the dilaton coupling for one of the three axions is given by $\frac{1}{2}\left(\vec{b}_{14}+\vec{b}_{23}\right)=\vec{\gamma}_{1}+\vec{\gamma}_{2}-\vec{\gamma}_{3}-\vec{\gamma}_{4}$. The other two sets of $N=16$ axions are then associated with the $\mathcal{F}_{(1) \pm}^{3}$ and $\mathcal{F}_{(1) \pm}^{4}$ pairs, with dilaton couplings given by $-\vec{\gamma}_{1}+\vec{\gamma}_{2}+\vec{\gamma}_{3}-\vec{\gamma}_{4}$ and $\vec{\gamma}_{1}-\vec{\gamma}_{2}+\vec{\gamma}_{3}-\vec{\gamma}_{4}$ respectively.

Comparing with the $T^{3}$ reduction of the heterotic string that we obtained previously, we find that the correspondence between the fields in the two descriptions can be summarised in the following Table:

\begin{tabular}{|c|c|c|c|c|c|}
\hline \multicolumn{3}{|c|}{ M-theory on K3 } & \multirow[b]{2}{*}{ Duality } & \multicolumn{2}{|c|}{ Heterotic string on $T^{3}$} \\
\hline$D=11$ & \multicolumn{2}{|c|}{$D=7$} & & $D=7$ & $D=10$ \\
\hline \multirow{8}{*}{$A_{(3)}$} & $A_{(3)}$, & $\vec{a}$ & $\longleftrightarrow$ & $A_{(2)}, \quad \vec{a}_{1}$ & \multirow[t]{4}{*}{$A_{(2)}$} \\
\hline & $A_{(1) 14}$, & & $\longleftrightarrow$ & $A_{(1) 2}, \quad \vec{a}_{12}$ & \\
\hline & $A_{(1) 24}$, & & $\longleftrightarrow$ & $A_{(1) 3}, \quad \vec{a}_{13}$ & \\
\hline & $A_{(1) 34}$, & $\vec{a}_{34}$ & $\longleftrightarrow$ & $A_{(1) 4}, \quad \vec{a}_{14}$ & \\
\hline & $A_{(1)}^{I}$ & $\vec{d}$ & $\longleftrightarrow$ & $B_{(1)}^{I}, \quad \vec{c}$ & $B_{(1)}^{I}$ \\
\hline & $A_{(1) 12}$, & & $\longleftrightarrow$ & $\mathcal{A}_{(1)}^{4}, \quad \vec{b}_{4}$ & \\
\hline & $A_{(1) 13}$, & $\vec{a}_{13}$ & $\longleftrightarrow$ & $\mathcal{A}_{(1)}^{3}, \quad \vec{b}_{3}$ & $G_{\mu \nu}$ \\
\hline & $A_{(1) 23}$, & $\vec{a}_{23}$ & $\longleftrightarrow$ & $\mathcal{A}_{(1)}^{2}, \quad \vec{b}_{2}$ & \\
\hline \multirow{7}{*}{$G_{\mu \nu}$} & $\overline{\mathcal{A}_{(0) \alpha}^{I}}$ & $\overline{\vec{b}_{\alpha}}$ & $\longleftrightarrow$ & $\begin{array}{ll}B_{(0) \alpha}^{I}, & \vec{c}_{\alpha}\end{array}$ & $\bar{B}_{(1)}^{I}$ \\
\hline & $\mathcal{A}_{(0) 4}^{1}$, & $\overrightarrow{b_{14}}$ & $\longleftrightarrow$ & $A_{(0) 34}, \quad \vec{a}_{134}$ & \multirow{3}{*}{$A_{(2)}$} \\
\hline & $\mathcal{A}_{(0) 4}^{2}$, & & $\longleftrightarrow$ & $A_{(0) 24}, \quad \vec{a}_{124}$ & \\
\hline & $\mathcal{A}_{(0) 4}^{3}$, & $\vec{b}_{34}$ & $\longleftrightarrow$ & $\underline{A_{(0) 23},} \quad \vec{a}_{123}$ & \\
\hline & $\mathcal{A}_{(0) 3}^{2}$, & $\vec{b}_{23}$ & $\longleftrightarrow$ & $\mathcal{A}_{(0) 4}^{3}, \quad \vec{b}_{34}$ & \multirow{3}{*}{$G_{\mu \nu}$} \\
\hline & $\overline{\mathcal{A}_{(0) 2}^{1}}$, & $\vec{b}_{12}$ & $\longleftrightarrow$ & $\overrightarrow{\mathcal{A}_{(0) 3}^{2}}, \quad \vec{b}_{23}$ & \\
\hline & $\overline{\mathcal{A}_{(0) 3}^{1}}$, & $\vec{b}_{13}$ & $\longleftrightarrow$ & $\overline{\mathcal{A}_{(0) 4}^{2}}, \quad \vec{b}_{24}$ & \\
\hline
\end{tabular}

Table 1: The correspondence between M-theory and the heterotic fields in $D=7$ 
In fact the fields $A_{(1) i j}$ and $\mathcal{A}_{(0) j}^{i}$ in the K3 compactification can be expressed in terms of the associated fields of the heterotic theory in a more covariant fashion by making use of the self-dual and anti-self-dual 2-forms $J_{ \pm}^{\alpha}$ defined in (5.2):

$$
\begin{aligned}
A_{(1) i j} & =\frac{1}{2}\left(J_{+i j}^{\alpha}+J_{-i j}^{\alpha}\right) A_{(1) \alpha}+\frac{1}{2}\left(J_{+i j}^{\alpha}-J_{-i j}^{\alpha}\right) \mathcal{A}_{(1)}^{\alpha} \\
\mathcal{A}_{(0) j}^{i} & =\frac{1}{4}\left(J_{+i j}^{\alpha}+J_{-i j}^{\alpha}\right) \epsilon_{\alpha \beta \gamma} A_{(0) \beta \gamma}+\frac{1}{4}\left(J_{+i j}^{\alpha}-J_{-i j}^{\alpha}\right) \epsilon_{\alpha \beta \gamma} \mathcal{A}_{(0) \gamma}^{\beta} .
\end{aligned}
$$

The four dilatons in the heterotic and M-theory reductions are related by an orthonormal transformation, $\vec{\phi}_{\mathrm{H}}=M \vec{\phi}_{\mathrm{M}}$, where

$$
M=\left(\begin{array}{cccc}
\frac{1}{8} & \frac{3}{8 \sqrt{7}} & \frac{1}{4} \sqrt{\frac{3}{7}} & \frac{\sqrt{15}}{4} \\
\frac{11}{8 \sqrt{7}} & -\frac{31}{56} & -\frac{31}{28 \sqrt{3}} & \frac{1}{4} \sqrt{\frac{5}{21}} \\
-\frac{5}{2 \sqrt{21}} & \frac{3}{14} \sqrt{3} & -\frac{31}{42} & \frac{1}{6} \sqrt{\frac{5}{7}} \\
-\frac{1}{2} \sqrt{\frac{5}{3}} & \frac{1}{2} \sqrt{\frac{15}{7}} & \frac{1}{6} \sqrt{\frac{5}{7}} & \frac{1}{6}
\end{array}\right) .
$$

This matrix satisfies $M^{5}=\mathbb{1}$. To understand the nature of this transformation, we note that both M-theory on K3 and the heterotic theory on $T^{3}$ have an $O(3,3) \sim S L(4, \mathbb{R})$ global symmetry as a subgroup of the full $O(3,19)$. The simple roots of $O(3,3)$ are given by the dilaton vectors of the underlined axionic fields listed in Table 1 . Thus we have

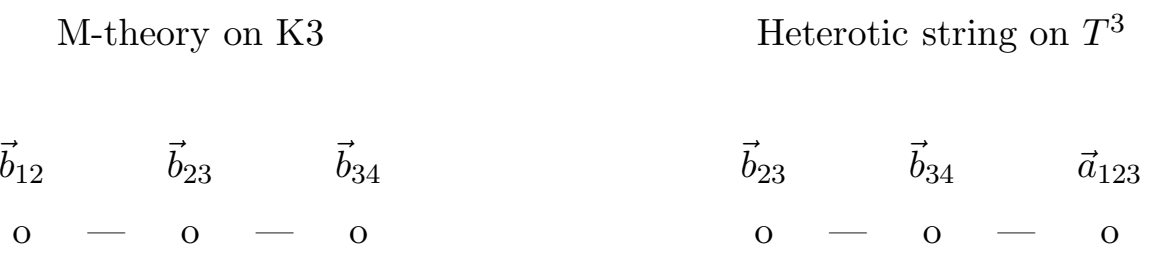

Figure 1. Simple roots of the $O(3,3)$ subgroup

The $O(3,3) \sim S L(4, \mathbb{R})$ group can be also viewed as a subgroup of the $S L(5, \mathbb{R})$ global symmetry group of maximal supergravity in $D=7$, which has simple roots $\vec{b}_{12}, \vec{b}_{23}, \vec{b}_{34}$ and $\vec{a}_{123}$. Thus we see that M-theory on K3 and the heterotic string on $T^{3}$ make two different truncations of $S L(5, \mathbb{R})$. From the maximal supergravity point of view, the two sets of simple roots of $O(3,3)$ are related by the Weyl group of $S L(5, \mathbb{R})$, which is $S_{5}$, the permutation group of five objects. Thus we would naturally expect that $M^{5}=\mathbb{1}$.

The matrix $M$ also maps the dilaton vector $\vec{a}=-\vec{g}$ of the 4 -form field strength $F_{(4)}$ in the M-theory reduction to $-\vec{a}_{1}$ of the 3 -form field strength $F_{(3)}$ in the heterotic string reduction. The minus sign is consistent with the fact that a dualisation of the 4-form field strength is necessary in order to make the identification of the two theories. Since the 
effective string coupling $\lambda_{7}$ of the heterotic string on $T^{3}$ is given by $\lambda_{7}=e^{-\frac{5}{8} \vec{a}_{1} \cdot \vec{\phi}}$, it follows from (5.8) that the seven-dimensional string coupling is [2]

$$
\lambda_{7}=V_{4}^{3 / 4}
$$

The complete set of mappings for all the dilaton vectors can be seen from Table 1 . Note that we have concentrated so far on establishing the relation between the dilaton couplings in the two theories; the detailed matching of the Kaluza-Klein modifications to the field strengths requires a more detailed analysis.

It is appropriate at this point to make a few remarks about the nature of the KaluzaKlein reduction procedure in the K3 compactification, and in particular to address the issue of the consistency of the reduction. In principle, the first step in any Kaluza-Klein compactification is to perform a harmonic expansion of all the higher-dimensional fields in terms of appropriate complete sets of scalar and tensor harmonics on the internal space, thereby arriving at a lower-dimensional theory with infinite towers of massive fields, together with finite numbers of massless fields. At this stage the reduction is guaranteed to be consistent, since one has done nothing more than a generalised Fourier expansion of the higher-dimensional fields.

In practice, one is usually interested in retaining only the finite number of massless fields arising from the Kaluza-Klein reduction. In other words, one would ideally wish to be able to set the infinite towers of massive fields to zero. The question then arises as to whether this is a consistent truncation of the lower-dimensional theory. In other words, is the setting to zero of the massive fields consistent with their own equations of motion? The dangers of inconsistency all stem from the non-linear interaction terms in the theory, which have the possibility in general of including "source terms" for the massive fields, built purely from the massless fields that are to be retained. Thus if we denote the massless fields generically by $\phi_{L}$, and the massive ones by $\phi_{M}$, a typical inconsistency would be signalled by the occurrence in the Lagrangian of non-linear interactions of the form $\phi_{M}\left(\phi_{L}\right)^{2}$, leading to equations of motion of the form

$$
\square \phi_{M}+M^{2} \phi_{M} \sim\left(\phi_{L}\right)^{2}
$$

which would not allow the massive fields to be set to zero.

In the simplest cases, such as Kaluza-Klein reduction on a torus, such dangerous terms cannot occur and so the truncation is indeed consistent. This follows from a simple grouptheoretic argument: if all the massless fields are singlets under some global symmetry group, 
while all the massive fields are non-singlets, then the massless fields cannot provide sources for the massive ones 40. In the toroidal case, the group in question is the global $U(1)^{n}$ symmetry of the $n$-torus. The massless fields are all uncharged, and are hence singlets under the $U(1)$ factors, while the massive fields are charged. To put it another way, products of the zero-mode harmonics on the torus (which are all constant) cannot generate non-zero-mode harmonics.

For the K3 reduction, the situation is less clear-cut. It would seem now to be quite conceivable that the product of zero-mode harmonics could generate non-zero-mode ones, since even the zero-mode harmonics are not now in general covariantly-constant. Correspondingly, one might expect that the massive fields could now have equations of motion of the form (5.19). It was argued in 41,42 that, in K3 or Calabi-Yau compactifications of supergravities, the source terms must in fact necessarily involve derivative couplings, of the generic form

$$
\square \phi_{M}+M^{2} \phi_{M} \sim\left(\partial \phi_{L}\right)^{2}
$$

and that there accordingly exists a regime of excitations of the massive fields where the energies are small compared to the Kaluza-Klein mass scale $M$, with the consequence that the inconsistencies in such reductions can then be neglected. One can expand (5.20) in such cases as

$$
\phi_{M} \sim M^{-2}\left(1+M^{-2} \square\right)^{-1}\left(\partial \phi_{L}\right)^{2} \sim M^{-2}\left(\partial \phi_{L}\right)^{2}+\cdots,
$$

Thus, at low energies the $\square \phi_{M}$ term in the massive field equation can be dropped, and the massive field $\phi_{M}$ can effectively be "integrated out," by substituting the solution $\phi_{M} \sim M^{-2}\left(\partial \phi_{L}\right)^{2}$ into the lower-dimensional Lagrangian. A related approach is to substitute the Kaluza-Klein reduction ansatz for the massless fields into the higher-dimensional Lagrangian, and then to integrate over the internal compactifying manifold.

Such an approximate discussion is applicable to situations where one is seeking to extract an effective low-energy "phenomenological" theory from the string compactification, where the mass scale $M$ of the Kaluza-Klein massive modes is very large compared with the energies of interest. However, it is not clear that this applicability extends to the regime of interest for non-perturbative duality symmetries. In particular, the conjectured duality between the heterotic string compactified on $T^{3}$ and M-theory compactified on K3 involves an inverse relation between the scale sizes of the $T^{3}$ and K3. Thus, to make any meaningful statements it is necessary to consider M-theory compactified on a large K3, where the Kaluza-Klein mass scale $M$ tends to zero, and here the neglect of kinetic terms for massive fields such as that in (5.20) becomes less and less innocent. Indeed, as $M$ tends to zero it 
is presumably more appropriate to expand (5.20) using not (5.21), but rather

$$
\phi_{M} \sim\left(1+M^{2} \square^{-1}\right)^{-1} \square^{-1}\left(\partial \phi_{L}\right)^{2} \sim \square^{-1}\left(\partial \phi_{L}\right)^{2}+\cdots
$$

Thus, rather than having the situation sketched in (5.21) where the effect of "integrating out" the massive modes is to modify the effective low-energy action by higher-order derivative couplings that are damped by inverse powers of the Kaluza-Klein mass scale, the effect now in the small- $M$ regime is to obtain non-derivative modifications with no damping.

It is therefore important in the context of M-theory/heterotic duality to try to establish whether or not the truncation to the massless sector is a consistent one. It is often asserted that the modulus space for Ricci-flat metrics on K3 is the coset space $O(3,19) /(O(3) \times O(19))$, and that this endows the seven-dimensional theory following from the K3 reduction of M-theory with a scalar manifold having this same coset structure. Indeed, it appears to be the case that if one substitutes the Kaluza-Klein ansatz for the zero-mode sector into the $D=11$ Lagrangian, and then integrates over K3, then the resulting seven-dimensional Lagrangian will have a scalar sector described by this $O(3,19) /(O(3) \times O(19))$ coset. However, as we have discussed above, it is a much more exacting and stringent question as to whether instead the substitution of the zero-mode ansatz into the eleven-dimensional field equations will be consistent with these fields' own equations of motion. Furthermore, although the effects of integrating out massive fields in the low-energy approximation (5.21) would not upset the coset structure of the Lagrangian for the lower-dimensional scalar fields, it is not so clear that this sigma-model structure would survive unscathed in the small- $M$ regime described by (5.22). As far as we are aware, this is a question that has not been addressed in the literature, and there appears to be no a priori argument that guarantees the consistency of the truncation. Although this could be argued to be a negligible problem in the context of low-energy phenomenology, it would seem to be a more significant one in the context of M-theory/heterotic duality, and it is deserving of further study. Indeed, one might argue that the consistency issue could provide a non-trivial test of the validity of the conjectured duality between M-theory and the heterotic string: Since the truncation of the $T^{3}$ compactification of the heterotic string to its massless sector is consistent, then the consistency of the truncation to the massless sector of the K3 compactification of M-theory would be a necessary consequence of the duality between the two theories. 


\section{Charge lattice relations}

In this section, we shall consider in detail the relation between the lattices of electric and magnetic charges that are allowed by the Dirac quantisation conditions in the K3 reduction of M-theory and the $T^{3}$ reduction of the heterotic string. It has been shown in Refs [14, 15, 16] that the minimum charges of M-branes can be fixed by invoking duality relations between M-theory, type IIA and type IIB string theories, together with the existence of certain "scale-setting" $p$-brane species [16]. In the case of M-theory, the charge units can also be fixed by consideration of the topological $\mathcal{L}_{F F A}$ term in the Lagrangian 443. We shall now show that the M-brane charges can also be fixed by consideration of the conjectured duality relation between M-theory compactified on K3 and heterotic string theory compactified on $T^{3}$, and we shall show that the results are consistent with the previous ones. We shall use the relations between charges under Kaluza-Klein dimensional reduction given in Ref./ [16]. To begin with, let us consider the heterotic string in ten dimensions. Making the gravitational constant $\kappa_{H}$ and the string tension $\alpha^{\prime}$ explicit, we may write the low-energy effective action as

$$
e^{-1} \mathcal{L}_{10}=\kappa_{H}^{-2}\left(R-\frac{1}{2}\left(\partial \phi_{1}\right)^{2}-\frac{1}{2} e^{\phi_{1}}\left(F_{(3)}\right)^{2}-\frac{1}{2} e^{\frac{1}{2} \phi_{1}} \operatorname{tr}\left(G_{(2)}\right)^{2}\right),
$$

where the $E_{8} \times E_{8}$ gauge fields $G_{(2)}$ are written in terms of gauge potentials as

$$
G_{(2)}=d B_{(1)}+\frac{1}{\sqrt{\alpha^{\prime}}}\left[B_{(1)}, B_{(1)}\right] .
$$

We shall first consider the subset of the theory corresponding to the $O(3,3)$-invariant subsector of the complete $D=7$ reduction; in other words, in the heterotic picture the Yang-Mills fields are not yet to be included. Equivalently, in the M-theory picture, the fields in $D=7$ associated with the "Eguchi-Hanson harmonics" on K3 are not yet to be included. Let us assume that the volume of $\mathrm{K} 3$ is given by $V_{4}=L_{1}^{4}$, whilst the volume of the $T^{3}$ in the heterotic picture is $V_{3}=L_{2}^{3}$. The duality of the two theories implies that

$$
\kappa_{7}^{2}=\frac{\kappa_{11}^{2}}{L_{1}^{4}}=\frac{\kappa_{H}^{2}}{L_{2}^{3}} .
$$

In $D=11$, the most general solution for the minimum M-brane charges, consistent with the Dirac quantisation condition, is given by

$$
Q_{e(4)}=(2 \pi)^{\alpha} \kappa_{11}^{4 / 3}, \quad Q_{m(4)}=(2 \pi)^{1-\alpha} \kappa_{11}^{2 / 3},
$$

where the $e$ and $m$ subscripts indicate electric and magnetic charges, and the (4) subscript indicates that they are carried by the 4 -form field strength. The constant $\alpha$ is as yet arbitrary. 
Upon reduction to $D=7$ on $\mathrm{K} 3$, in the $O(3,3)$ subset the 4 -form reduces to $F_{(4)}$ and $F_{(2) i j}$. It follows that their charges are given by

$$
Q_{m(4)}=(2 \pi)^{1-\alpha} \kappa_{11}^{2 / 3}, \quad Q_{m(2) i j}=(2 \pi)^{1-\alpha} \kappa_{11}^{2 / 3} L_{1}^{-2}
$$

(We need only list either electric or magnetic charges, since these are related by the Dirac quantisation condition.)

In the heterotic picture, on the other hand, the $O(3,3)$ subset of fields is obtained from the $T^{3}$ reduction of the pure $N=1$ supergravity multiplet. The most general solutions for the $D=10$ string and 5-brane charges are given by

$$
Q_{e(3)}=(2 \pi)^{\beta} \kappa_{H}^{3 / 2}, \quad Q_{m(3)}=(2 \pi)^{1-\beta} \kappa_{H}^{1 / 2}
$$

Upon making a $T^{3}$ reduction, the 3 -form gives rise to $F_{(3)}$ and $F_{(2) \alpha}$, together with 1-form field strengths for axions, which we shall not consider here. In addition, there are three Kaluza-Klein 2-forms $\mathcal{F}_{(2)}^{\alpha}$. The charges of these fields are given by

$$
Q_{e(3)}=(2 \pi)^{\beta} \kappa_{H}^{3 / 2} L_{2}^{-3}, \quad Q_{m(2) \alpha}=(2 \pi)^{1-\beta} \kappa_{H}^{1 / 2} L_{2}^{-1}, \quad Q_{m(2) \alpha}^{\mathrm{KK}}=L_{2} .
$$

In each case, the corresponding magnetic or electric dual charges are related by the $D=7$ Dirac quantisation conditions. Note that the magnetic Kaluza-Klein charge is associated with a NUT charge, and hence its charge unit is determined by topological considerations.

The duality between M-theory reduced on K3 and the heterotic theory reduced on $T^{3}$ implies that the charges carried by the various fields in the two pictures should be equated, in accordance with the equivalences of the corresponding fields as given in Table 1. This gives rise to three independent equations:

$$
(2 \pi)^{\beta} \kappa_{H}^{3 / 2} L_{2}^{-3}=(2 \pi)^{1-\alpha} \kappa_{11}^{2 / 3}, \quad(2 \pi)^{1-\beta} \kappa_{H}^{1 / 2} L_{2}^{-1}=L_{2}=(2 \pi)^{1-\alpha} \kappa_{11}^{2 / 3} L_{1}^{-2} .
$$

Together with the relation (6.3) between the gravitational constants, we find that

$$
\begin{aligned}
\kappa_{11}^{2}=\frac{1}{2 \pi} L_{1}^{6} L_{2}^{3}, & \kappa_{H}^{2}=\frac{1}{2 \pi} L_{1}^{2} L_{2}^{6}, \\
\alpha=\frac{2}{3}, & (2 \pi)^{4 \beta}=(2 \pi)^{3} L_{1}^{2} L_{2}^{-2} .
\end{aligned}
$$

From these, we see that the M-brane charges in $D=11$ are completely determined, and must be given by

$$
Q_{e(4)}=n\left(2 \pi \kappa_{11}^{2}\right)^{2 / 3}, \quad Q_{m(4)}=m\left(2 \pi \kappa_{11}^{2}\right)^{1 / 3}
$$

where $n$ and $m$ are integers. This conclusion is the same as that obtained in [16], where T-duality between the type IIA and type IIB theories was invoked. 
We now turn to a consideration of the Yang-Mills sector. The $E_{8} \times E_{8}$ gauge potentials may be expanded in terms of the Cartan-subalgebra generators $H_{I}$ and the non-zero root generators $E_{a}$ as $B_{(1)}=B_{(1)}^{I} H_{I}+B_{(1)}^{a} E_{a}$. In particular we see that with respect to the Cartan subalgebra gauge potentials, the coupling of the non-zero root potentials takes the form $d B_{(1)}^{a} E_{a}+\left(\alpha^{\prime}\right)^{-1 / 2} B_{(1)}^{I} \wedge B_{(1)}^{a}\left[H_{I}, E_{a}\right]=\left(d B_{(1)}^{a}+\left(\alpha^{\prime}\right)^{-1 / 2} \alpha^{I a} B_{(1)}^{I} \wedge B_{(1)}^{a}\right) E_{a}$. Thus the fields $B_{(1)}^{a}$ interact with the $U(1)^{16}$ Cartan subalgebra potentials via minimal coupling, with the 16 electric charges given in terms of the components of the root vectors $\vec{\alpha}_{a}$. The basic units of electric Yang-Mills charge in the ten-dimensional heterotic string are therefore given in terms of the 16 simple-root vectors $\vec{\alpha}_{i}$ of $E_{8} \times E_{8}$, since all the other Yang-Mills charges are expressible in terms of linear combinations of these with integer coefficients. To make this more precise, we choose to define the magnetic charges by the integrals

$$
Q_{m}^{I} \equiv \int G_{(2)}^{I}
$$

It follows from the form of the covariant derivative $D B_{(1)}^{i}=d B_{(1)}^{i}+\left(\alpha^{\prime}\right)^{-1 / 2} \alpha^{I i} B_{(1)}^{I} \wedge$ $B_{(1)}^{i}$ that the Dirac quantisation conditions will require the magnetic charges to lie on the reciprocal lattice

$$
\vec{Q}_{m}=m_{i} \sqrt{\alpha^{\prime}} \vec{\mu}^{i}
$$

where $m_{i}$ are integers and $\vec{\mu}^{i}$ are the fundamental weight vectors, defined by $\vec{\alpha}_{i} \cdot \vec{\mu}^{j}=\delta_{i}^{j}$.

For comparison, we now consider the charge lattice for the Yang-Mills sector coming from the reduction of M-theory on K3. In particular, we consider the charges under the 16 abelian 2-form fields arising from the 16 anti-self-dual harmonic 2-forms on K3 which, in the approximate discussion of section 5, were associated with the 16 Eguchi-Hanson instantons. These are the fields that are conjectured to be related by duality to the Cartan subalgebra of the $E_{8} \times E_{8}$ symmetry of the heterotic string.

We may derive the charge lattice for these 16 fields in the M-theory picture by using an abstract description of the cohomology of K3. Specifically, one may introduce a set of sixteen 2 -forms $\sigma_{(2)}^{i}$ that are "dual" to the sixteen anti-self-dual harmonics $\tilde{\omega}_{(2) i}$, in the sense that that

$$
\int_{K 3} \sigma_{(2)}^{i} \wedge \tilde{\omega}_{(2) j}=\delta_{j}^{i}
$$

The 2-forms $\sigma_{(2)}^{i}$ are normalised so that their integrals over the relevant 162 -cycles $\Sigma_{i}$ in $\mathrm{K} 3$ are given by

$$
L_{1}^{2} \int_{\Sigma_{i}} \sigma_{(2)}^{j}=\delta_{i}^{j}
$$

(We are taking the harmonic 2-forms $\tilde{\omega}_{(2) i}$ to have the dimensions of (Length) ${ }^{2}$, and the dual 2 -forms $\sigma_{(2)}^{i}$ to have dimensions (Length) ${ }^{-2}$. We use the dimensionful parameter $L_{1}=V_{4}^{1 / 4}$ 
that we introduced earlier, where $V_{4}$ is the volume of $\mathrm{K} 3$, in order to balance the dimensions.) Then, one has the result that 31]

$$
\int_{K 3} \tilde{\omega}_{i} \wedge \tilde{\omega}_{j}=L_{1}^{4} M_{i j}
$$

where $M_{i j}$ is the Cartan matrix of $E_{8} \times E_{8}$. This is given in terms of the simple root vectors $\vec{\alpha}_{i}$ by $M_{i j}=\vec{\alpha}_{i} \cdot \vec{\alpha}_{j}$. From the above equations, we easily see that if we expand $\tilde{\omega}_{(2) i}$ in terms of $\sigma_{(2)}^{i}$, we have $\tilde{\omega}_{(2) i}=M_{j i} \sigma_{(2)}^{j}$, and hence that

$$
\int_{\Sigma_{i}} \tilde{\omega}_{(2) j}=L_{1}^{2} M_{i j}
$$

The 2-forms $\tilde{\omega}_{(2) i}$ do not have the appropriate normalisation for giving canonical diagonal kinetic terms for the associated spacetime 2-form field strengths in the Kaluza-Klein reduction of $F_{(4)}$. To obtain the proper kinetic terms, we should now define new linear combinations $\omega_{(2)}^{I}$ by

$$
\omega_{(2)}^{I}=\mu^{I i} \tilde{\omega}_{(2) i}
$$

where $\mu^{I i}$ denotes the set of 16 components of the fundamental weight vectors $\vec{\mu}^{i}$ that we introduced previously. The 2 -forms $\omega_{(2)}^{I}$ therefore satisfy the relations

$$
\int_{K 3} \omega_{(2)}^{I} \wedge \omega_{(2)}^{J}=L_{1}^{4} \delta^{I J}, \quad \int_{\Sigma_{i}} \omega_{(2)}^{I}=L_{1}^{2} \alpha_{i}^{I} .
$$

Performing the Kaluza-Klein reduction

$$
F_{(4)}=F_{(2)}^{I} \omega_{(2)}^{I},
$$

we see from (6.18) that the normalisation of the $\omega_{(2)}^{I}$ implies that the kinetic terms for the spacetime 2-forms $F_{(2)}^{I}$ will be canonical. The magnetic charge $Q_{m(4)}$ in $D=11$ for a 5-brane wrapped around the 2 -cycle $\Sigma_{i}$ in K3 will therefore be given by

$$
Q_{m(4)}=\int F_{(4)}=Q_{m}^{I} \int_{\Sigma_{i}} \omega_{(2)}^{I}=L_{1}^{2} Q_{m}^{I} \alpha_{i}^{I}
$$

where $Q_{m}^{I}=\int F_{(2)}^{I}$ is the magnetic charge of the resulting 3-brane in $D=7$. (Recall that $L_{1}$ is the length scale-factor for K3 with volume $V_{4}=L_{1}^{4}$.) The 5 -brane charges in $D=11$ have already been determined in (6.10), however. Thus it follows that consistency with (6.20) requires that the magnetic 3 -brane charges in $D=7$ lie on the lattice

$$
\vec{Q}_{m}=m_{i} \vec{\mu}^{i} Q_{0}
$$

\footnotetext{
${ }^{6}$ It is worth noting that there is an alternative basis for the 16 Eguchi-Hanson type anti-self-dual harmonic 2 -forms $\tilde{\omega}_{i}$, in which we have $\int_{\Sigma_{i}} \tilde{\omega}_{j}=L_{1}^{2} \delta_{i j}$ and (6.15), rather than (6.16) and (6.15). In this alternative basis, (6.21) is replaced by $\vec{Q}_{m}=n_{i} \vec{a}_{i} Q_{0}$, where $n_{i}$ are integers. This is entirely equivalent to (6.21), since the integers $n_{i}$ are related to the integers $m_{i}$ in (6.21) by $n_{i}=\left(M^{-1}\right)^{i j} m_{j}$, and both $M_{i j}$, the Cartan matrix of $E_{8} \times E_{8}$, and its inverse $\left(M^{-1}\right)^{i j}$, have entirely integer-valued components.
} 
where $\vec{Q}_{m}=\left(Q_{m}^{1}, Q_{m}^{2}, \ldots, Q_{m}^{16}\right)$ and

$$
Q_{0}=\left(2 \pi \kappa_{11}^{2}\right)^{1 / 3}\left(\frac{\kappa_{H}^{2}}{2 \pi \kappa_{11}^{4}}\right)^{1 / 5}
$$

Note that the lattice of magnetic charges (6.21), derived from the K3 compactification of Mtheory, is of the same form as the lattice (6.12) that we obtained in the $T^{3}$ compactification of the heterotic string. A precise identification then requires that $\alpha^{\prime}=Q_{0}^{2}$.

\section{$7 \quad p$-brane orbits with Yang-Mills charges}

In the previous section, we looked at the duality relation between the heterotic string compactified on $T^{3}$ and M-theory compactified on K3. As well as being a relation that holds in the low-energy effective field theories, this should also be seen at the level of BPS states in the two full theories. For example, the M5-brane wrapped around the K3 manifold is dual to a vertical reduction of the NS-NS string of the heterotic theory, while the vertical reduction of the M2-brane is dual to the NS-NS 5-brane wrapped around the volume of $T^{3}$.

To study this, let us focus on the spectrum of particles, and their 3-brane duals, in seven dimensions. From the M-theory point of view, they arise from membranes and 5-branes wrapping around the 2-cycles of K3. In our discussion given in section 5, we encountered two different types of 2-cycle in the construction that we were using for K3, namely six 2-cycles corresponding to the usual non-contractible 2-surfaces in $T^{4}$, and sixteen additional 2-cycles, each associated with one of the sixteen Eguchi-Hanson instantons used to smooth out the conical singularities on the identified 4-torus. ¿From the field-theoretic point of view, the concept of "wrapping" a $p$-brane soliton around a particular $m$-cycle in the internal space essentially translates into the idea of constructing a $(p-m)$-brane soliton supported by the lower-dimensional field arising in the Kaluza-Klein expansion from the harmonic form associated to the given $m$-cycle on the internal space. When $m$ is less than the dimension of the internal manifold, part of the transverse space of the $p$-brane also becomes internal. In a toroidal reduction, this notion of vertical reduction can be made mathematically precise, by "stacking" $p$-branes along the reduction axes. No such analogous procedure has yet been implemented for K3 or Calabi-Yau compactifications, where the internal coordinates correspond both to the world-volume and the transverse space of the wrapped $p$-brane. (Such an implementation would presumably first of all require that one know the explicit metric on the K3 or Calabi-Yau internal space.)

When the membrane wraps around any of the six 4-torus 2-cycles, it gives a corresponding set of six 0-branes that are supported by the six 2-form field strengths coming from the 
Kaluza-Klein reduction of the $D=114$-form on $T^{4}$. These correspond, in the heterotic picture, to the six 0-branes that describe the three winding and three Kaluza-Klein modes on the compactifying 3 -torus.

When the membrane wraps instead around any of the sixteen 2-cycles associated with the Eguchi-Hanson manifolds, it should give rise to sixteen 0-branes that are supported by the associated 2-form field strengths which, in the heterotic picture, come from the $T^{3}$ dimensional reduction of the Yang-Mills fields of the $D=10$ heterotic string. However, a standard construction of a $p$-brane supported by one of the Yang-Mills fields leads to a solution that is not supersymmetric. ${ }^{7}$ The reason why such a $p$-brane is non-supersymmetric is that the supersymmetry transformation rule for the gauginos is of the form $\delta \lambda^{I} \sim G_{\mu \nu}^{I} \Gamma^{\mu \nu} \epsilon$, and the matrix $G_{\mu \nu}^{I} \Gamma^{\mu \nu}$ is non-degenerate in the case of the standard ansatz for one of the fields $G_{(2)}^{I}$.

On the other hand, we know that the Cartan-subalgebra $U(1)^{16}$ subset of the YangMills fields, together with the Kaluza-Klein and winding vector fields, form an irreducible multiplet in the fundamental representation of $O(10-D, 26-D)$. Thus naively one might expect that starting from a supersymmetric $p$-brane supported either by a Kaluza-Klein vector or by a winding vector, one could rotate to a solution supported purely by the YangMills fields. However, it turns out that this is not the case; the reason is that solutions supported only by the Yang-Mills fields would lie on different $O(10-D, 26-D)$ orbits from those supported by a single Kaluza-Klein or winding vector field. In this section we shall study the various possible types of orbit that can arise, in order to see on which orbits supersymmetric solutions supported purely by the Yang-Mills fields can lie.

\section{1 $D=9$ black holes and Yang-Mills wave excitations}

The key issues can be adequately illustrated by considering the nine-dimensional theory arising from the dimensional reduction of $N=1$ supergravity in $D=10$ coupled to a single $D=10$ Maxwell multiplet. This subset of the heterotic-theory fields has a global $O(1,2)$ invariance in $D=9$. The bosonic sector of the Lagrangian is given by (2.2), with the index $I$ on the Yang-Mills fields $B_{(1)}^{I}$ and $B_{(0)}^{I}$ taken to have one value only. We shall denote the associated potentials by $B_{(1)}$ and $B_{(0)}$. The Lagrangian is

$$
e^{-1} \mathcal{L}_{9}=R-\frac{1}{2}(\partial \phi)^{2}-\frac{1}{2}(\partial \varphi)^{2}-\frac{1}{2} e^{\sqrt{2} \varphi}\left(\partial B_{(0)}\right)^{2}-\frac{1}{12} e^{-\sqrt{\frac{8}{7}} \phi}\left(F_{(3)}\right)^{2}
$$

\footnotetext{
${ }^{7}$ By the "standard construction" we mean that one starts with a Lagrangian of the form $e^{-1} \mathcal{L}=R-$ $\frac{1}{2}(\partial \phi)^{2}-e^{a \phi} F^{2}$, and makes a metric ansatz of the usual form $d s^{2}=e^{2 A(r)} d x^{\mu} d x_{\mu}+e^{2 B(r)}\left(d r^{2}+r^{2} d \Omega^{2}\right)$, with $F$ either of the form $F=Q \Omega$ (for a magnetic solution) or of the dual form (for an electric solution).
} 


$$
-\frac{1}{4} e^{-\sqrt{\frac{2}{7}} \phi}\left(e^{\sqrt{2} \varphi}\left(F_{(2)}\right)^{2}+e^{-\sqrt{2} \varphi}\left(\mathcal{F}_{(2)}\right)^{2}+\left(G_{(2)}\right)^{2}\right)
$$

where the various field strengths, following (A.10), are given by

$$
\begin{aligned}
& \mathcal{F}_{(2)}=d \mathcal{A}_{(1)}, \quad G_{(2)}=d B_{(1)}+B_{(0)} d \mathcal{A}_{(1)}, \quad F_{(2)}=d A_{(1)}+B_{(0)} d B_{(1)}+\frac{1}{2}\left(B_{(0)}\right)^{2} d \mathcal{A}_{(1)}, \\
& F_{(3)}=d A_{(2)}+\frac{1}{2} B_{(1)} d B_{(1)}-\frac{1}{2} \mathcal{A}_{(1)} d A_{(1)}-\frac{1}{2} A_{(1)} d \mathcal{A}_{(1)} .
\end{aligned}
$$

We can construct extremal 2-charge electric 0-brane or magnetic 5-brane solutions using the Kaluza-Klein and winding vectors $\mathcal{A}_{(1)}$ and $A_{(1)}$. In this subsection, we shall look at the electrically-charged situation, and shall consider extremal black-hole solutions. The non-vanishing fields in this case are given by

$$
\begin{array}{ll}
d s_{9}^{2}=\left(H_{1} H_{2}\right)^{-6 / 7} d x^{\mu} d x_{\mu}+\left(H_{1} H_{2}\right)^{1 / 7}\left(d r^{2}+r^{2} d \Omega_{7}^{2}\right), \\
e^{-\sqrt{14} \phi}=H_{1} H_{2}, \quad e^{\sqrt{2} \varphi}=\frac{H_{1}}{H_{2}}, \\
A_{(1)}= \pm H_{1}^{-1} d t, \quad \mathcal{A}_{(1)}= \pm H_{2}^{-1} d t, \quad B_{(1)}=0,
\end{array}
$$

where the harmonic functions are given by

$$
H_{1}=1+\frac{q_{1}}{r^{6}}, \quad H_{2}=1+\frac{q_{2}}{r^{6}} .
$$

The \pm signs on the 1-form potentials in (7.3) are independent, and reflect the fact that the bosonic equations are quadratic in field strengths, and hence solutions exist for any choice of signs. The electric charges $Q_{1}$ and $Q_{2}$ are given by

$$
Q_{1}= \pm q_{1}, \quad Q_{2}= \pm q_{2}
$$

The mass of the black hole is given by

$$
m=q_{1}+q_{2} .
$$

Although the bosonic theory has four solutions, corresponding to the four different sign choices in (7.3), the supersymmetry transformations depend linearly on the field strengths, and, consequently, not all of the four sign choices for the charges need yield supersymmetric solutions. In the case of maximal supergravities, on the other hand, the fraction of preserved supersymmetry is independent of the sign choices for similar 2-charge solutions. This can be seen from the fact that the 32 eigenvalues of the Bogomol'nyi matrix are 39] $\mu=$

\footnotetext{
${ }^{8}$ For simplicity, we are considering single-centre isotropic solutions here. The discussion that follows can be immediately generalised to multi-centre solutions, where $H_{i}=1+\sum_{a} \frac{q_{i}^{a}}{\left|\vec{y}-\vec{y}_{a}^{i}\right|}$, with the potential $\omega$ written in terms of $* d H_{i}$ in the standard way.
} 
$m \pm Q_{1} \pm Q_{2}$, with each of the four sign combinations occurring with multiplicity 8. Thus in maximal supergravity, the 2-charge solutions always preserve $\frac{1}{4}$ of the supersymmetry, regardless of the choice of signs. On the other hand, in $N=1$ nine-dimensional supergravity the 16 eigenvalues of the corresponding Bogomol'nyi matrix are a subset of the 32 given above. Specifically, they are given by

$$
\mu=m \pm\left(Q_{1}+Q_{2}\right)
$$

with each sign choice occurring with multiplicity 8 . This means that in the $N=1$ theory, only two out of the four sign choices in (7.5) give solutions that preserve supersymmetry, namely

$$
\left(Q_{1}, Q_{2}\right)=\left(q_{1}, q_{2}\right), \quad \text { or } \quad\left(Q_{1}, Q_{2}\right)=\left(-q_{1},-q_{2}\right)
$$

In each of these cases, the 2-charge solution preserves $\frac{1}{2}$ of the $N=1$ supersymmetry. For the remaining two sign choices in (7.5), all of the supersymmetry is broken. From now on, we shall consider just the supersymmetric solutions, and shall concentrate on the first of the two cases listed in (7.8). Note that when $Q_{1}=Q_{2}$ the two harmonic functions $H_{1}$ and $\mathrm{H}_{2}$ become equal, and the solution reduces to a "single-charge" solution supported by the single 2-form field strength of pure $N=1$ supergravity in $D=9$ [44].

There are in total three electric 2-form charges $Q_{1}, Q_{2}$ and $Q_{3}$ in the nine-dimensional theory that we are considering, associated with the vectors $A_{(1)}, \mathcal{A}_{(1)}$ and $B_{(1)}$ respectively. As we saw in section 2.1, these vectors form a triplet under the $O(1,2)$ global symmetry group. The associated invariant quadratic form constructed from the charges is

$$
\mathcal{I}=Q_{3}^{2}+Q_{Y}^{2}-Q_{X}^{2}=Q_{3}^{2}-2 Q_{1} Q_{2}
$$

where $Q_{X}=\frac{1}{\sqrt{2}}\left(Q_{1}+Q_{2}\right), Q_{Y}=\frac{1}{\sqrt{2}}\left(Q_{1}-Q_{2}\right)$, as can be seen from the discussion in section 2.1. The orbits on the three-dimensional charge lattice that are filled out by acting on a given solution are thus characterised by the quadratic invariant $\mathcal{I}$. In particular, there are three inequivalent types of orbit, corresponding to the cases where $\mathcal{I}$ is positive, negative or zero.

To see the nature of the three types of orbit in detail, we first note that a single-charge solution supported either by $Q_{1}$ or by $Q_{2}$ belongs to a light-like orbit of $O(1,2)$, for which $\mathcal{I}$ vanishes. On the other hand, if $Q_{1}$ and $Q_{2}$ are both non-vanishing, and of the same sign, the orbit is time-like, with $\mathcal{I}<0$. If instead these two charges have opposite signs, then the orbit is space-like, with $\mathcal{I}>0$.

Such solutions with light-like or time-like orbits have curvature singularities which, for positive-mass solutions, lie on the horizon at $r=0$, but they are free of singularities 
outside the horizon. On the other hand solutions lying on space-like orbits necessarily have naked singularities that lie outside the horizon. This is because the charges are of opposite sign, and hence the supersymmetry requirement (7.8) implies that one of the harmonic functions will have a negative coefficient for its $r^{-6}$ term. Although one might be tempted to dismiss such solutions from consideration, their presence is necessary in order to complete the charge lattice. Indeed, a single-charge solution supported exclusively by the Yang-Mills charge $Q_{3}$ would lie on such a spacelike orbit. Moreover, the fundamental $D=9$ Yang-Mills field excitations of the theory corresponding to spontaneously broken Yang-Mills generators must lie in short massive supermultiplets that carry such charges. Given the presumed correspondence between such excitations and classical particle or wave solutions, one would expect to find classical solutions with these charges. The fact that massive supermultiplets carrying Yang-Mills charges must necessarily be short multiplets gives a hint that their origin may be sought in massless wave-like super Yang-Mills solutions in ten dimensions. Indeed, as we shall now show, they do originate from such wave-like solutions, and moreover, this helps explain the origin of the naked singularities.

In fact, the naked singularity in the 2-charge solution (7.3) with $Q_{1}=q_{1}>0$ and $Q_{2}=q_{2}<0$ is purely an artefact of dimensional reduction. If we oxidise the solution back to $D=10$, the metric becomes

$$
d s_{10}^{2}=H_{1}^{-3 / 4}\left(-H_{2}^{-1} d t^{2}+H_{2}\left(d z+\left(H_{2}^{-1}-1\right) d t\right)^{2}\right)+H_{1}^{1 / 4} d \vec{y} \cdot d \vec{y} .
$$

This is the intersection of a string, associated with the harmonic function $H_{1}$, and a gravitational pp-wave, associated with $\mathrm{H}_{2}$. The string has no associated naked singularity since $H_{1}$ has a positive coefficient in its $r^{-6}$ term. Although $r^{-6}$ in $H_{2}$ has a negative coefficient, this does not necessarily imply the existence of a naked singularity in the associated wave solution.

To study this issue, let us concentrate on a particular solution lying on the space-like charge orbit which has a particularly simple ten-dimensional interpretation. If we act on the 2-charge solution $(7.3)$ with the $O(1,2)$ transformation

$$
\Lambda=\left(\begin{array}{ccc}
1 & 0 & 0 \\
0 & \cos \theta & \sin \theta \\
0 & -\sin \theta & \cos \theta
\end{array}\right),
$$

where $\tan \frac{1}{2} \theta=\left(-q_{1} / q_{2}\right)^{1 / 2}$, we obtain a new solution with the same metric as in (7.3), but with the other fields now given by

$$
e^{-\sqrt{14} \phi}=H_{1} H_{2}, \quad e^{-\sqrt{2} \varphi}=H_{1} H_{2}, \quad B_{0}=\frac{1}{\sqrt{2}}\left(H_{2}-H_{1}\right) \sin \theta,
$$




$$
\begin{aligned}
& A_{(1)}=\left(\left(H_{1} H_{2}\right)^{-1}-H_{1}^{-1}-H_{2}^{-1}\right) d t, \quad \mathcal{A}_{(1)}=-\left(H_{1} H_{2}\right)^{-1} d t \\
& B_{(1)}=\frac{1}{\sqrt{2}} \sin \theta\left(H_{1}^{-1}-H_{2}^{-1}\right) d t .
\end{aligned}
$$

Oxidising this up to $D=10$, we obtain the solution

$$
\begin{aligned}
d s_{10}^{2} & =-\left(H_{1} H_{2}\right)^{-1} d t^{2}+\left(H_{1} H_{2}\right)\left(d z+\left(H_{1} H_{2}\right)^{-1} d t\right)^{2}+d \vec{y}^{2} \\
\phi_{1} & =0, \quad B_{(1)}=\frac{1}{\sqrt{2}} \sin \theta\left(H_{2}-H_{1}\right) d z, \quad A_{(2)}=\text { pure gauge } .
\end{aligned}
$$

Note in particular that $F_{(3)}=0$.

This solution describes a wave-like excitation of the Yang-Mills field, propagating along the $z$ direction. It can be compared with the general class of wave-like solutions in the heterotic string, described in [48]. There, solutions were sought of the form

$$
\begin{aligned}
& d s_{10}^{2}=-2 d u(d v-W(u, \vec{y}) d u)^{2}+d \vec{y}^{2}, \\
& B_{1}=M(u, \vec{y}) d u, \quad A_{(2)}=b(u, \vec{y}) \wedge d u, \quad \phi_{1}=0 .
\end{aligned}
$$

For the class of solutions that are of interest to us, $b(u, \vec{y})$ is zero, and $M(u, \vec{y})$ depends only on $\vec{y}$. From the results of 48], one has it that the equations of motion are satisfied provided

$$
\partial_{i} \partial_{i} M=0, \quad \partial_{i} \partial_{i} W=-\partial_{i} M \partial_{i} M
$$

Thus, $M$ is harmonic on the transverse space, and $W$ can be solved by taking $W=\alpha M-$ $\frac{1}{2} M^{2}$. If $M$ is the harmonic function $c r^{-6}$, then the wave solution (7.14) is equivalent to (7.13) under the following identifications:

$$
u=\frac{z}{\sqrt{2}}, \quad v=\frac{2 t-z}{\sqrt{2}}, \quad c=\sqrt{-2 q_{1} q_{2}}, \quad \alpha=\frac{q_{1}+q_{2}}{\sqrt{-2 q_{1} q_{2}}} .
$$

Although the $O(1,2)$ global symmetry can rotate the charges so that the Kaluza-Klein electric charge $Q_{2}$ vanishes, one finds that the Kaluza-Klein vector $\mathcal{A}_{(1)}$ can never become of pure gauge form upon rotation from any non-trivial starting value. This implies that if a generic $O(1,2)$ rotation of the solution (7.3) is oxidised to $D=10$, the metric will necessarily have a wave-like character; it is given by

$$
d s_{10}^{2}=\left(a^{2} H_{1}+b^{2} H_{2}\right)^{-3 / 4}\left(-2 d t+\frac{H_{1} H_{2}}{a^{2} H_{1}+b^{2} H_{2}} d z\right) d z+\left(a^{2} H_{1}+b^{2} H_{2}\right)^{1 / 4}, d \vec{y}^{2},
$$

where $a$ and $b$ are constants arising from the $O(1,2)$ rotation. For generic $a$ and $b$, this solution describes the intersection of a wave and a string. The linear combination $a^{2} H_{1}+$ $b^{2} H_{2}$ becomes the harmonic function associated with the string.

It is worth emphasising that the solutions with unavoidable naked singularities in $D=9$ arise in the case where the quadratic invariant (7.9) is positive, i.e. where the charge orbit is 
space-like. In [21], it was shown that for perturbative fundamental single-string excitations, the above quadratic invariant $\mathcal{I}$ is bounded above, lying in the interval f $^{\text {}}-\infty<\mathcal{I}<1$. At the level of supergravity particle solutions, on the other hand, there is no such upper bound. This is because supergravity solutions can describe not only single-string excitations but also multiple-string excitations. The algebraic classification of heterotic string states into single-string and multiple-string spectra remains an interesting open problem.

\subsection{5 -branes in $D=9$}

Now we turn to the nine-dimensional 5-brane solution, which is given by

$$
\begin{aligned}
& d s_{9}^{2}=\left(H_{1} H_{2}\right)^{-1 / 7} d x^{\mu} d x_{\mu}+\left(H_{1} H_{2}\right)^{6 / 7}\left(d r^{2}+r^{2} d \Omega_{2}^{2}\right), \\
& e^{\sqrt{14} \phi}=H_{1} H_{2}, \quad e^{\sqrt{2} \varphi}=\frac{H_{2}}{H_{1}}, \\
& A_{(1)}=Q_{1} \omega, \quad \mathcal{A}_{(1)}=Q_{2} \omega, \quad B_{(1)}=0,
\end{aligned}
$$

where the harmonic functions are given by

$$
H_{1}=1+\frac{q_{1}}{r}, \quad H_{2}=1+\frac{q_{2}}{r},
$$

and $d \omega=\Omega_{(2)}$ is the volume form on the unit 2-sphere. (Again, for simplicity we are considering single-centre isotropic solutions here, which could easily be generalised to multicentre solutions.) The mass per unit 4-volume of the 5-brane is given by

$$
m=q_{1}+q_{2}
$$

In terms of the two individual parameters $q_{1}$ and $q_{2}$, there exist 5 -brane solutions where the magnetic charges under the Kaluza-Klein and winding vectors are

$$
Q_{1}= \pm q_{1}, \quad Q_{2}= \pm q_{2}
$$

As in the case of electric black holes discussed earlier, the supersymmetry requires that $\left(Q_{1}, Q_{2}\right)=\left(q_{1}, q_{2}\right)$ or $\left(Q_{1}, Q_{2}\right)=\left(-q_{1},-q_{2}\right)$.

To construct a 5-brane solution supported purely by the Yang-Mills 2-form in $D=9$, let us begin with the solution (7.18), for which $Q_{1}=q_{1}$ and $Q_{2}=q_{2}$ are arbitrary, with $Q_{1} Q_{2}<0$, while $Q_{3}=0$. In order to map the charges to a configuration where $Q_{1}=Q_{2}=0$, while $Q_{3}$ is non-zero, we must act with a matrix in an $O(1,1)$ subgroup of $O(1,2)$ as follows:

$$
\left(\begin{array}{c}
\frac{1}{\sqrt{2}}\left(Q_{1}+Q_{2}\right) \\
\frac{1}{\sqrt{2}}\left(Q_{1}-Q_{2}\right) \\
0
\end{array}\right) \rightarrow\left(\begin{array}{c}
0 \\
0 \\
Q_{3}
\end{array}\right)=\left(\begin{array}{ccc}
\cosh t & \sinh t & 0 \\
0 & 0 & -1 \\
\sinh t & \cosh t & 0
\end{array}\right)\left(\begin{array}{c}
\frac{1}{\sqrt{2}}\left(Q_{1}+Q_{2}\right) \\
\frac{1}{\sqrt{2}}\left(Q_{1}-Q_{2}\right) \\
0
\end{array}\right)
$$

\footnotetext{
${ }^{9}$ Note that our sign convention is the opposite of the one used in 21.
} 
The parameter $t$ is therefore given by

$$
e^{2 t}=-\frac{Q_{2}}{Q_{1}}
$$

Again, we see that $Q_{1}$ and $Q_{2}$ must have opposite signs in order for the mapping to be possible. Applying this transformation to the fields $X, Y$ and $Z$ introduced in section 2.1, one can determine the transformed expressions for the dilaton $\varphi$ and the axion $B_{(0)}$. Similarly, by transforming the column vector $\left(A_{(1)}, \mathcal{A}_{(1)}, B_{(1)}\right)$, one obtains the expressions for the transformed vector potentials. Upon doing this, we find that the solution (7.18) becomes

$$
\begin{aligned}
& d s_{9}^{2}=\left(H_{1} H_{2}\right)^{-1 / 7} d x^{\mu} d x_{\mu}+\left(H_{1} H_{2}\right)^{6 / 7}\left(d r^{2}+r^{2} d \Omega_{2}^{2}\right), \\
& e^{\sqrt{14} \phi}=H_{1} H_{2}, \quad e^{\sqrt{2} \varphi}=\frac{1}{2} \sqrt{-\frac{Q_{1} H_{2}}{Q_{2} H_{1}}}+\frac{1}{2} \sqrt{-\frac{Q_{2} H_{1}}{Q_{1} H_{2}}}, \\
& \frac{1}{\sqrt{2}} B_{(0)}=\frac{Q_{1} H_{2}+Q_{2} H_{1}}{Q_{1} H_{2}-Q_{2} H_{1}}, \\
& A_{(1)}=0, \quad \mathcal{A}_{(1)}=0, \quad B_{(1)}=Q_{1} \sqrt{-\frac{2 Q_{2}}{Q_{1}}} \omega .
\end{aligned}
$$

The harmonic functions retain their original form, and can in general describe multi-centre solutions. In the single-centre isotropic case, they are given by (7.19). In this special case, the dilaton $\varphi$ and the axion $B_{(0)}$ can be rewritten as

$$
e^{-2 \sqrt{2} \varphi}=-\frac{4 Q_{1} Q_{2}}{\left(Q_{1}-Q_{2}\right)^{2}} H_{1} H_{2}, \quad \frac{1}{\sqrt{2}} B_{(0)}=\frac{Q_{1} H_{2}+Q_{2} H_{1}}{Q_{1}-Q_{2}} .
$$

In line with our previous discussion we see indeed that this solution, which carries only the charge $Q_{3}$ of the Yang-Mills field strength $G_{(2)}$, is not of the form of a "standard" single-charge $p$-brane solution. It is nevertheless, of course, supersymmetric, since we have obtained it by performing an $O(1,2)$ rotation on a standard supersymmetric solution. Although it involves only a single Yang-Mills charge $Q_{3}=q_{1} \sqrt{-2 q_{2} / q_{1}}$, it has two independent parameters $q_{1}$ and $q_{2}$ associated with the two harmonic functions $H_{1}$ and $H_{2}$ given in (7.19). However, the two parameters $q_{1}$ and $q_{2}$ must both be non-zero and of opposite signs, implying that there is again a naked singularity, at $r=\left|\min \left(q_{1}, q_{2}\right)\right|$. If $q_{1}=-q_{2}$, the 5-brane has zero mass. The connection between naked singularities and masslessness has been extensively discussed in the context of BPS black holes of the $D=4$ toroidallycompactified heterotic string in Refs [49,50]. A similar phenomenon also occurs in $p$-brane solitons in maximal supergravity [45], including massless dyonic strings in $D=6$ [51]. The occurrence of a naked singularity means that the naive relation between zero eigenvalues of the Bogomol'nyi matrix and unbroken supersymmetries no longer holds, and in particular 
the apparent enhancement of supersymmetry in the massless limit does not in actuality occur [52].

It is interesting to look at the form of the solution (7.24) when oxidised back to $D=10$. We find that it becomes

$$
\begin{aligned}
& d s_{10}^{2}=e^{\frac{1}{4 \sqrt{2}} \varphi}\left(\left(H_{1} H_{2}\right)^{-1 / 8}\left(d x^{\mu} d x_{\mu}+e^{-\sqrt{2} \varphi} d z^{2}\right)+\left(H_{1} H_{2}\right)^{7 / 8}\left(d r^{2}+r^{2} d \Omega_{2}^{2}\right)\right) \\
& e^{2 \phi_{1}}=e^{\frac{1}{\sqrt{2}} \varphi}\left(H_{1} H_{2}\right)^{-1 / 2}, \quad A_{(2)}=Q_{1} \sqrt{-\frac{Q_{2}}{Q_{1}}}\left(\frac{Q_{1} H_{2}+Q_{2} H_{1}}{Q_{1} H_{2}-Q_{2} H_{1}}\right) \omega \wedge d z \\
& B_{(1)}=Q_{1} \sqrt{-\frac{2 Q_{2}}{Q_{1}}} \omega+\sqrt{2}\left(\frac{Q_{1} H_{2}+Q_{2} H_{1}}{Q_{1} H_{2}-Q_{2} H_{1}}\right) d z
\end{aligned}
$$

where $e^{\sqrt{2} \varphi}$ is the function given in $(7.24)$. Note that in $D=10$ the solution also involves the NS-NS 3-form field strength. As usual, we give the metric in the Einstein frame here. In terms of the string frame, this becomes

$$
d s_{\mathrm{str}}^{2}=d x^{\mu} d x_{\mu}+e^{-\sqrt{2} \varphi} d z^{2}+\left(H_{1} H_{2}\right)\left(d r^{2}+r^{2} d \Omega_{2}^{2}\right) .
$$

In the above discussion, we have considered the case where we began with a 2-charge solution with $Q_{1}=q_{1}$ and $Q_{2}=q_{2}$ opposite in sign. By doing so, we ensured that the solution was supersymmetric, but at the price of its having a naked singularity. We could instead have started from a 2-charge solution with charges $Q_{1}=q_{1}$ and $Q_{2}=-q_{2}$, again taken to be opposite in sign. In this case, the solution is non-supersymmetric, however, since now all the Bogomol'nyi eigenvalues in (7.7) are non-zero. However, if $Q_{1}$ and $-Q_{2}$ are both positive then the solution will be free from naked singularities. This solution can also be rotated to one that carries only the Yang-Mills charge $Q_{3}$. The form of this solution is identical to (7.24), except that the replacement of $\left(Q_{1}, Q_{2}\right)=\left(q_{1}, q_{2}\right)$ by $\left(Q_{1}, Q_{2}\right)=\left(q_{1},-q_{2}\right)$ implies that the harmonic function $H_{2}$ is given by $1-Q_{2} / R$ rather than $1+Q_{2} / r$. In particular, this means that when $Q_{1}=-Q_{2}$, and hence $H_{1}=H_{2}$, the solution (7.24) is now a "standard" single-charge 5-brane solution, supported by the Yang-Mills field strength $G_{(2)}$. In line with our earlier discussion, this is indeed non-supersymmetric.

This analysis of $p$-brane orbits supported by 2 -form field strengths in the toroidallycompactified heterotic string theory can be easily generalised to lower dimensions. In $D \geq 6$, the global symmetry group is $O(10-D, 26-D)$, and the vector potentials form a $(36-2 D)$ dimensional representation. The orbits are characterised by an invariant quadratic form that generalises (7.9), and fall into the three categories of time-like, space-like and light-like orbits. Any single-charge solution supported by a Kaluza-Klein vector or a winding vector lies on a light-like orbit. A two-charge solution involving both a Kaluza-Klein vector and 
a winding vector can lie either on a time-like or a space-like orbit: When the two charges are of the same sign, and hence the solution has no naked singularity, the orbit is time-like. It does not cover the points in the charge lattice where the solution involves only charges that are carried by the Yang-Mills 2-forms. On the other hand, when the two charges are of opposite signs, and so the solution suffers from a naked singularity, the orbit is spacelike and the solution can be rotated to one supported purely by Yang-Mills field strengths. All the above solutions preserve half of the supersymmetry of the theory. (There are also time-like and space-like orbits for non-supersymmetric two-charge solutions. They can be obtained from the supersymmetric 2-charge solutions by reversing the relative sign of the two charges but keeping the mass unchanged. This phenomenon of supersymmetry being broken as a consequence of a sign change of a charge, while keeping the mass fixed, occurs also in maximal supergravities, but only for solutions with more than 3 charges [45, 46, 47].)

In $D=5$ there is an additional 2-form field strength that comes from the dualisation of the NS-NS 3 -form field. This is a singlet under the $O(5,21)$ global symmetry, and there exists a 3-charge solution involving this singlet, a Kaluza-Klein vector, and a winding vector. In $D=4$, the global symmetry is enlarged to $O(6,24) \times S L(2, \mathbb{R})$, and the theory allows 4-charge solutions. The solution space in this case has been extensively studied $53,54,55,56,57$.

In this section, we have been principally interested in supersymmetric solutions supported purely by the Yang-Mills fields, in order to make contact with M-theory compactified on K3. As we have discussed, such solutions suffer from naked singularities. The origin of these naked singularities in the M-theory picture is less clear. We have seen that when an M-brane wraps around any of the sixteen Eguchi-Hanson 2-cycles in K3, it gives rise to a $p$-brane which, in the heterotic picture, is supported by a Yang-Mills field. As we have seen in section 5, these 2-cycles can shrink to zero size, in which case the wrapped M-brane will become massless [58]. Indeed, the $p$-brane supported by the Yang-Mills field in the heterotic picture can also become massless by adjusting the Yang-Mills moduli, i.e. the expectation values of the Cartan-subalgebra scalar fields.

\section{Acknowledgments}

We are grateful to P.S. Aspinwall, G.W. Gibbons, C.J. Isham, R. Kallosh and A. Sen for useful discussions. H.L. and K.S.S. thank Texas A\&M University and SISSA, and H.L. and C.N.P. thank CERN, for hospitality. 


\section{Appendices}

\section{A Toroidal reduction of the heterotic string}

Our starting point is the low-energy effective Lagrangian for the bosonic sector of the tendimensional heterotic string. In this paper, we have considered the duality relations of the dimensionally reduced and "fully Higgsed" heterotic string. That is, after dimensional reduction, we have considered vacuum configurations in which the adjoint-representation scalars arising in the dimensional reduction are assigned general expectation values corresponding to vanishing potential energy. Such a general Higgs vacuum causes a spontaneous breakdown of the $E_{8} \times E_{8}$ Yang-Mills gauge symmetry to its maximal torus subgroup $U(1)^{16}$, whose algebra coincides with the Cartan subalgebra of $E_{8} \times E_{8}$. It is thus the set of dimensional reductions of this $U(1)^{16}$ Abelian Yang-Mills supergravity that principally concern us here. For simplicity, we may begin by retreating to $D=10$ and replacing the full Yang-Mills-supergravity theory by its Abelian $U(1)^{16}$ maximal-torus sub-theory. The bosonic sector of this $D-10$ Lagrangian is given by [59]

$$
e^{-1} \mathcal{L}_{10}=R-\frac{1}{2}\left(\partial \phi_{1}\right)^{2}-\frac{1}{12} e^{\phi_{1}}\left(F_{(3)}\right)^{2}-\frac{1}{4} e^{\frac{1}{2} \phi_{1}} \sum_{I}\left(G_{(2)}^{I}\right)^{2}
$$

where $G_{(2)}^{I}=d B_{(1)}^{I}$ are the sixteen $U(1)$ gauge field strengths, and $F_{(3)}=d A_{(2)}+\frac{1}{2} B_{(1)}^{I} \wedge d B_{(1)}^{I}$. The dilaton $\phi$ in the standard convention is in fact equal to $-\phi_{1}$.

We perform the toroidal reduction using the notation and conventions of 39, 5]. The metric is reduced according to the ansatz

$$
d s_{10}^{2}=e^{\vec{s} \cdot \vec{\phi}} d s_{D}^{2}+\sum_{\alpha=2}^{11-D} e^{2 \vec{\gamma}_{\alpha} \cdot \vec{\phi}}\left(h^{\alpha}\right)^{2},
$$

where $\vec{\gamma}_{\alpha}=\frac{1}{2} \vec{s}-\frac{1}{2} \vec{f}_{\alpha}$ and

$$
\begin{aligned}
& \vec{s}=\left(s_{2}, s_{3}, \ldots s_{11-D}\right), \quad \vec{f}_{\alpha}=(\underbrace{0,0, \ldots, 0}_{\alpha-2},(10-\alpha) s_{\alpha}, s_{\alpha+1}, s_{\alpha+2}, \ldots, s_{11-D}), \\
& s_{\alpha}=\sqrt{\frac{2}{(10-\alpha)(9-\alpha)}}, \quad 2 \leq \alpha \leq 11-D .
\end{aligned}
$$

The vectors $\vec{s}$ and $\vec{f}_{\alpha}$ satisfy

$$
\vec{s} \cdot \vec{s}=\frac{10-D}{4(D-2)}, \quad \vec{s} \cdot \vec{f}_{\alpha}=\frac{2}{D-2}, \quad \vec{f}_{\alpha} \cdot \vec{f}_{\beta}=2 \delta_{\alpha \beta}+\frac{2}{D-2} .
$$

Here, we are using $\vec{\phi}$ to denote the $(10-D)$-component vector $\vec{\phi}=\left(\phi_{2}, \phi_{3}, \ldots, \phi_{11-D}\right)$. Note that we have reserved $\phi_{1}$ as the (negative of) the original dilaton in $D=10$. The 
quantities $h^{\alpha}$ are given by

$$
h^{\alpha}=d z^{\alpha}+\mathcal{A}_{(1)}^{\alpha}+\mathcal{A}_{(0) \beta}^{\alpha} d z^{\beta}=\tilde{\gamma}_{\beta}^{\alpha}\left(d z^{\beta}+\hat{\mathcal{A}}_{(1)}^{\beta}\right),
$$

where $\tilde{\gamma}_{\beta}^{\alpha}=\delta_{\beta}^{\alpha}+\mathcal{A}_{(0) \beta}^{\alpha}$, and $\gamma^{\alpha}{ }_{\beta}$ is the inverse of $\tilde{\gamma}_{\beta}^{\alpha}$ [39, 5 . We have also introduced the redefined Kaluza-Klein vectors $\hat{\mathcal{A}}_{1}^{\alpha}=\gamma^{\alpha}{ }_{\beta} \mathcal{A}_{(1)}^{\beta}$. Note that $\gamma^{a}{ }_{\beta}$ and $\tilde{\gamma}^{\alpha}{ }_{\beta}$ are non-zero only when $\alpha \leq \beta$.

Applying the above reduction ansatz, we obtain the $D$-dimensional Lagrangian

$$
\begin{aligned}
e^{-1} \mathcal{L}_{D}= & R-\frac{1}{2}(\partial \vec{\phi})^{2}-\frac{1}{12} e^{\vec{a}_{1} \cdot \vec{\phi}}\left(F_{(3)}\right)^{2}-\frac{1}{4} \sum_{\alpha} e^{\vec{a}_{1 \alpha} \cdot \vec{\phi}}\left(F_{(2) \alpha}\right)^{2} \\
& -\frac{1}{2} \sum_{\alpha<\beta} e^{\vec{a}_{1 \alpha \beta} \cdot \vec{\phi}}\left(F_{(1) \alpha \beta}\right)^{2}-\frac{1}{4} \sum_{I} e^{\vec{c} \cdot \vec{\phi}}\left(G_{(2)}^{I}\right)^{2}-\frac{1}{2} \sum_{\alpha, I} e^{\vec{c}_{\alpha} \cdot \vec{\phi}}\left(G_{(1) \alpha}^{I}\right)^{2} \\
& -\frac{1}{4} \sum_{\alpha} e^{\vec{b}_{\alpha} \cdot \vec{\phi}}\left(\mathcal{F}_{(2)}^{\alpha}\right)^{2}-\frac{1}{2} \sum_{\alpha<\beta} e^{\vec{b}_{\alpha \beta} \cdot \vec{\phi}}\left(\mathcal{F}_{(1) \beta}^{\alpha}\right)^{2} .
\end{aligned}
$$

The indices $\alpha, \beta \ldots$ run from 2 to $11-D$. The various field strengths here are given by

$$
\begin{aligned}
F_{(3)}= & d A_{(2)}+\frac{1}{2} B_{(1)}^{I} d B_{(1)}^{I}-\left(d A_{(1) \alpha}+\frac{1}{2} B_{(0) \alpha}^{I} d B_{(1)}^{I}+\frac{1}{2} B_{(1)}^{I} d B_{(0) \alpha}^{I}\right) \hat{\mathcal{A}}_{(1)}^{\alpha} \\
& +\frac{1}{2}\left(d A_{(0) \alpha \beta}-B_{(0)[\alpha}^{I} d B_{(0) \beta]}^{I}\right) \hat{\mathcal{A}}_{(1)}^{\alpha} \hat{\mathcal{A}}_{(1)}^{\beta}, \\
F_{(2) \alpha}= & \gamma^{\beta}{ }_{\alpha}\left(d A_{(1) \beta}+\frac{1}{2} B_{(0) \beta}^{I} d B_{(1)}^{I}+\frac{1}{2} B_{(1)}^{I} d B_{(0) \beta}^{I}+\left(d A_{(0) \beta \gamma}-B_{(0)[\beta}^{I} d B_{(0) \gamma]}^{I}\right) \hat{\mathcal{A}}_{(1)}^{\gamma}\right), \\
F_{(1) \alpha \beta}= & \gamma^{\gamma}{ }_{\alpha} \gamma^{\delta}{ }_{\beta}\left(d A_{(0) \gamma \delta}-B_{(0)[\gamma}^{I} d B_{(0) \delta]}^{I}\right), \\
G_{(2)}^{I}= & d B_{(1)}^{I}-d B_{(0) \alpha}^{I} \hat{\mathcal{A}}_{(1)}^{\alpha}, \\
G_{(1) \alpha}^{I}= & \gamma^{\beta}{ }_{\alpha} d B_{(0) \beta}^{I}, \quad \mathcal{F}_{(2)}^{\alpha}=\tilde{\gamma}_{\beta}^{\alpha} d \hat{\mathcal{A}}_{(1)}^{\beta}, \quad \mathcal{F}_{(1) \beta}^{\alpha}=\gamma_{\beta}^{\gamma} d \mathcal{A}_{(0) \gamma}^{\alpha},
\end{aligned}
$$

In the Lagrangian (A.6), we have augmented the (10-D)-component vector $\vec{\phi}$ introduced in (A.2) by appending $\phi_{1}$ as its first component, so that now $\vec{\phi}=\left(\phi_{1}, \phi_{2}, \ldots, \phi_{11-D}\right)$. The dilaton vectors $\vec{a}_{1}, \ldots$ characterise the couplings of the dilatonic scalars $\vec{\phi}$ to the various field strengths; their details can be found in [39,5], and they are given by

$$
\begin{aligned}
& \vec{a}_{1}=(1,-2 \vec{s}), \quad \vec{a}_{1 \alpha}=\left(1, \vec{f}_{\alpha}-2 \vec{s}\right), \quad \vec{a}_{1 \alpha \beta}=\left(1, \vec{f}_{\alpha}+\vec{f}_{\beta}-2 \vec{s}\right), \\
& \vec{b}_{\alpha}=\left(0,-\vec{f}_{\alpha}\right), \quad \vec{b}_{\alpha \beta}=\left(0,-\vec{f}_{\alpha}+\vec{f}_{\beta}\right), \\
& \vec{c}=\left(\frac{1}{2},-\vec{s}\right)=\frac{1}{2} \vec{a}_{1}, \quad \vec{c}_{\alpha}=\left(\frac{1}{2}, \vec{f}_{\alpha}-\vec{s}\right) .
\end{aligned}
$$

These dilaton vectors $\vec{c}_{\alpha}$ satisfy the orthogonality property $\vec{c}_{\alpha} \cdot \vec{c}_{\beta}=2 \delta_{\alpha \beta}$. The dot product of $\vec{a}_{1}$ or $\vec{c}$ with $\vec{b}_{\alpha \beta}$ vanishes. In fact $\vec{b}_{\alpha \beta}$ can also be written as $\vec{b}_{\alpha \beta}=-\vec{c}_{\alpha}+\vec{c}_{\beta}$. For the 3-form $F_{(3)}$ and its dimensional reductions $F_{(2) \alpha}$ and $F_{(1) \alpha \beta}$, the dilaton vectors $\vec{a}_{1}, \vec{a}_{1 \alpha}$ and $\vec{a}_{1 \alpha \beta}$ are precisely those introduced in [39,5] for the dimensional reduction of the 4-form field $F_{(4)}$ in $D=11$ supergravity. (The 3-form $F_{(3)}$ here coincides with the NS-NS 3-form of type 
IIA supergravity, which arises as the first-step reduction of $F_{(4)}$.) The dilaton vectors $\vec{b}_{\alpha}$ and $\vec{b}_{\alpha \beta}$, for the Kaluza-Klein vectors $\mathcal{A}_{(1)}^{\alpha}$ and the Kaluza-Klein axions $\mathcal{A}_{(0) \beta}^{\alpha}$ also coincide precisely with the ones introduced in [39,5]. The reason for these relations to the dilaton vectors arising from the reduction of $D=11$ supergravity is that the pure supergravity sector of the heterotic theory can be obtained as a truncation of type IIA supergravity where the R-R fields are set to zero. Finally, the dilaton vectors $\vec{c}$ and $\vec{c}_{\alpha}$ for the Yang-Mills fields $G_{(2)}^{I}$ and their dimensional reductions $G_{(1) \alpha}^{I}$ come from reducing 2-form field strengths in $D=10$ to 2 -forms and 1 -forms in $D$ dimensions.

It is advantageous at this stage to perform some field redefinitions on the 2-form and 1-form vector potentials, in order to simplify the structure of the equations. Thus we define

$$
\begin{aligned}
B_{(1)}^{I} & =B^{\prime I}{ }_{(1)}+B_{(0) \alpha}^{I} \hat{\mathcal{A}}_{(1)}^{\alpha}, \\
A_{(1) \alpha} & =A_{(1) \alpha}^{\prime}-A_{(0) \alpha \beta} \hat{\mathcal{A}}_{(1)}^{\beta}+\frac{1}{2} B_{\alpha}^{I} B_{(1)}^{I I}, \\
A_{(2)} & =A_{(2)}^{\prime}+\frac{1}{2} A_{(0) \alpha \beta} \hat{\mathcal{A}}_{(1)}^{\alpha} \hat{\mathcal{A}}_{(1)}^{\beta}+\frac{1}{2} B_{(0) \alpha}^{I} B_{(1)}^{I} \hat{\mathcal{A}}_{(1)}^{\alpha}+\frac{1}{2} A_{(1) \alpha}^{\prime} \hat{\mathcal{A}}_{(1)}^{\alpha} .
\end{aligned}
$$

In terms of these, the 3 -form and 2-form field strengths become

$$
\begin{aligned}
F_{(3)} & =d A_{(2)}^{\prime}+\frac{1}{2} B_{(1)}^{I} d B_{(1)}^{I I}-\frac{1}{2} \hat{\mathcal{A}}_{(1)}^{\alpha} d A_{(1) \alpha}^{\prime}-\frac{1}{2} A_{(1) \alpha}^{\prime} d \hat{\mathcal{A}}_{(1)}^{\alpha}, \\
F_{(2) \alpha} & =\gamma_{\alpha}^{\beta}\left(d A_{(1) \beta}^{\prime}-A_{(0) \beta \gamma} d \hat{\mathcal{A}}_{(1)}^{\gamma}+B_{(0) \beta}^{I} d B_{(1)}^{\prime I}+\frac{1}{2} B_{(0) \beta}^{I} B_{(0) \gamma}^{I} d \hat{\mathcal{A}}_{(1)}^{\gamma}\right) \\
G_{(2)}^{I} & =d B_{(1)}^{\prime I}+B_{(0) \alpha}^{I} d \hat{\mathcal{A}}_{(1)}^{\alpha} \\
\mathcal{F}_{(2)}^{\alpha} & =\tilde{\gamma}^{\alpha}{ }_{\beta} d \hat{\mathcal{A}}_{(1)}^{\beta} .
\end{aligned}
$$

The expressions for the 1-form field strengths $F_{(1) \alpha \beta}, \mathcal{F}_{(1) \beta}^{\alpha}$ and $G_{(1) \alpha}^{I}$ are unchanged, and still given by (A.7).

\section{B Further coset geometry examples}

In section 2.2 of this paper, we gave a geometrical construction of the class of cosets (2.11), which includes those arising in the toroidal dimensional reduction of ten-dimensional simple supergravity coupled to $N$ abelian gauge fields. In this appendix, we extend this construction to several further classes of cosets. Since the principles of the construction are closely parallel to those discussed in section 2.2, we shall just present the essential features of the examples.

We begin with an additional class of cosets in which the matrix $W$ is real, and satisfies the pseudo-orthogonality condition $W^{\mathrm{T}} \eta W=\eta$. Then we can have an antisymmetricmatrix embedding: 
$\underline{W^{\mathrm{T}} \eta W=\eta, \quad W^{\mathrm{T}}=-W}$

The numerator group here is generated by matrices $\Lambda$ satisfying $\Lambda^{\mathrm{T}} \eta \Lambda=\eta$, whose action on $W$ is $W \rightarrow \Lambda^{\tau} W \Lambda$. It is easily seen that, without loss of generality, the fiducial matrix $W_{0}$ can be taken to have the antisymmetric block diagonal form

$$
W_{0}=\operatorname{diag}(\sigma, \sigma, \ldots, \sigma)
$$

where $\sigma$ is the $2 \times 2$ antisymmetric matrix

$$
\sigma=\left(\begin{array}{cc}
0 & 1 \\
-1 & 0
\end{array}\right)
$$

It is then evident that this matrix can only satisfy the given conditions if $\eta$ has the form

$$
\eta=\operatorname{diag}(\underbrace{-1,-1, \ldots,-1}_{2 p}, \underbrace{1,1, \ldots, 1}_{2 q})
$$

Thus we see that the numerator group is $O(2 p, 2 q)$, while the stability subgroup is given by $O(2 p, 2 q)$ matrices $\Lambda$ that satisfy not only $\Lambda^{\mathrm{T}} \eta \Lambda=\eta$ but also $\Lambda^{\mathrm{T}} W_{0} \Lambda=W_{0}$. It is not hard to see that with these conditions, the matrices $\Lambda$ lie in $U(p, q)$. Thus we obtain that the $O(2 p, 2 q)$ orbits span the coset space

$$
\frac{O(2 p, 2 q)}{U(p, q)}
$$

For the next examples, we consider two classes of coset where $W$ is real, but now leaves invariant the antisymmetric metric $\Omega$ defined by

$$
\Omega=\operatorname{diag}(\sigma, \sigma, \ldots, \sigma)
$$

where $\sigma$ is given by (B.2). In these cases the numerator group is generated by matrices $\Lambda$ that satisfy $\Lambda^{\mathrm{T}} \Omega \Lambda=\Omega$, and whose action on $W$ is $W \rightarrow \Lambda^{\mathrm{T}} W \Lambda$. We first consider a symmetric-matrix embedding:

$\underline{W^{\mathrm{T}} \Omega W=\Omega, \quad W^{\mathrm{T}}=W}$

In this case, the numerator group is defined by matrices $\Lambda$ satisfying $\Lambda^{\mathrm{T}} \Omega \Lambda=\Omega$, and thus it is $S p(2 n, \mathbb{R})$. It is easy to see that the fiducial matrix $W_{0}$ can be taken to be diagonal, and of the form

$$
W_{0}=(\underbrace{-1,-1, \ldots,-1}_{2 p}, \underbrace{1,1, \ldots, 1}_{2 q}) .
$$


(The individual numbers of - and + signs must necessarily both be even, in order that $W_{0}$ be able to satisfy the given conditions.) The stability subgroup is given by matrices $\Lambda$ that satisfy both $\Lambda^{\mathrm{T}} \Omega \Lambda=\Omega$ and $\Lambda^{\mathrm{T}} W_{0} \Lambda=W_{0}$. These are the same conditions as in the previous example, and so again we find that the stability subgroup to be $U(p, q)$. Thus we obtain the coset

$$
\frac{S p(2 p+2 q, \mathbb{R})}{U(p, q)} .
$$

Next, consider the antisymmetric-matrix embedding:

$\underline{W^{\mathrm{T}} \Omega W=\Omega, \quad W^{\mathrm{T}}=-W}$

The numerator group is the same as for the symmetric-matrix embedding above. The fiducial matrix should be taken to be antisymmetric in this case; we may pick

$$
W_{0}=\operatorname{diag}(\underbrace{-\sigma,-\sigma, \ldots,-\sigma}_{p}, \underbrace{\sigma, \sigma, \ldots, \sigma}_{q}) .
$$

The stability subgroup is defined by matrices $\Lambda$ satisfying both $\Lambda^{\mathrm{T}} \Omega \Lambda=\Omega$ and $\Lambda^{\mathrm{T}} W_{0} \Lambda=$ $W_{0}$, and it is thus clearly $S P(2 p, \mathbb{R}) \times S P(2 q, \mathbb{R})$. The coset in this case is therefore

$$
\frac{S p(2 p+2 q, \mathbb{R})}{S p(2 p, \mathbb{R}) \times S p(2 q, \mathbb{R})} .
$$

Continuing on with metric-preserving numerator groups, we can now consider cases where the numerator group is generated by complex matrices $\Lambda$ that preserve the sesquilinear form $\Lambda^{\dagger} \eta \Lambda=\eta$. These act on complex matrices $W$ according to $W \rightarrow \Lambda^{\dagger} W \Lambda$. Without loss of generality, $\eta$ can be taken to be symmetric. Thus we may consider a Hermitean embedding:

$\underline{W^{\dagger} \eta W=\eta, \quad W^{\dagger}=W:}$

Here, the metric $\eta$ has the form

$$
\eta=\operatorname{diag}(\underbrace{-1,-1, \ldots,-1}_{p}, \underbrace{1,1, \ldots, 1}_{q}) .
$$

Note that there is no loss of generality in taking $W$ to be Hermitean rather than antiHermitean, since the latter is related to the former by a multiplication by i. The fiducial matrix can be taken to be

$$
W_{0}=\operatorname{diag}(\underbrace{-1,-1, \ldots,-1}_{m}, \underbrace{1,1, \ldots, 1}_{p-m}, \underbrace{-1,-1, \ldots,-1}_{n}, \underbrace{1,1, \ldots, 1}_{q-m}) .
$$


The numerator group is $U(p, q)$, and its stability subgroup is $U(m, q-n) \times U(p-m, n)$. Thus we have the coset

$$
\frac{U(p, q)}{U(m, q-n) \times U(p-m, n)} .
$$

As a final set of examples, we consider two cases where the numerator group is not metric-preserving. Specifically, we shall take it to be $S L(n, \mathbb{R})$, defined by $n \times n$ real matrices that satisfy $\operatorname{det} \Lambda=1$. Their action on $W$ is $W \rightarrow \Lambda^{\mathrm{T}} W \Lambda$. We can then take the coset matrices $W$ to satisfy either $W^{\mathrm{T}}=W$ or $W^{\mathrm{T}}=-W$ :

$\underline{W^{\mathrm{T}}}=W$

The fiducial matrix $W_{0}$ can be taken to be

$$
W_{0}=\operatorname{diag}(\underbrace{-1,-1, \ldots,-1}_{p}, \underbrace{1,1, \ldots, 1}_{q})
$$

It follows that the stability subgroup will be the subgroup of $S L(p+q, \mathbb{R})$ matrices that satisfy $\Lambda^{\mathrm{T}} W_{0} \Lambda=W_{0}$; in other words it will be $S O(p, q)$. Thus we have the coset

$$
\frac{S L(p+q, \mathbb{R})}{S O(p, q)} .
$$

$\underline{W^{\mathrm{T}}=-W}$

The fiducial matrix $W_{0}$ can be taken to be

$$
W_{0}=\operatorname{diag}(\sigma, \sigma, \ldots, \sigma)
$$

The stability subgroup will therefore be generated by the subset of $S L(2 n, \mathbb{R})$ matrices that satisfy $\Lambda^{\mathrm{T}} W_{0} \Lambda=W_{0}$; in other words it will be $\operatorname{Sp}(2 n, \mathbb{R})$. Thus we have the coset structure

$$
\frac{S L(2 n, \mathbb{R})}{S p(2 n, \mathbb{R})}
$$

\section{References}

[1] C.M. Hull and P.K. Townsend, Unity of superstring dualities, Nucl. Phys. B294 (1995) 196, hep-th/9410167.

[2] E. Witten, String theory dynamics in various dimensions, Nucl. Phys. B443 (1995) 85, hep-th/9503124. 
[3] P. Hořava and E. Witten, Nucl. Phys. B460 (1996) 506.

[4] P. Horăva and E. Witten, Nucl. Phys. B475 (1996) 94.

[5] E. Cremmer, B. Julia, H. Lü and C.N. Pope, Dualisation of dualities, Nucl. Phys. B523 (1998) 73, hep-th/9710119.

[6] E. Cremmer, H. Lü, C.N. Pope and K.S. Stelle, Spectrum-generating symmetries for BPS solitons, Nucl. Phys. B520 (1998) 132, hep-th/9707207.

[7] K. Iwasawa, Ann. Math. 50 (1949) 507.

[8] D.V. Alekseevskii, Math. USSR Izvestija, 9 (1975), No.2.

[9] L. Andrianopoli, R. D'Auria, S. Ferrara, P. Fré and M. Trigiante, R-R scalars, U-duality and solvable Lie algebras, Nucl. Phys. B496 (1997) 617, hep-th/9611014.

[10] L. Andrianopoli, R. D'Auria, S. Ferrara, P. Fré, R. Minasian, M. Trigiante, Solvable Lie algebras in type IIA, type IIB and $M$ theories, Nucl. Phys. B493 (1997) 249, hep-th/9612202.

[11] L. Castellani, A. Ceresole, R. D'Auria, S. Ferrara, P. Fré and M. Trigiante, G/H Mbranes and $A d S_{p}+2$ geometries, hep-th/9803039.

[12] G. Arcioni, A. Ceresole, F. Cordaro. R. D'Auria, P. Fré, L. Gualtieri, M. Trigiante, $N=8$ BPS black holes with $1 / 2$ or $1 / 4$ supersymmetry and solvable Lie algebra decomposition, hep-th/9807136.

[13] D.N. Page, A physical picture of the K3 gravitational instanton, Phys. Lett. B80 (1978) 55.

[14] J.H. Schwarz, The power of M-theory, Phys. Lett. B367 (1996) 97, hep-th/9510086.

[15] S.P. de Alwis, A note on brane tension and M-theory, Phys. Lett. B388 (1996) 291, hep-th/9607011.

[16] M.S. Bremer, H. Lü, C.N. Pope and K.S. Stelle, Dirac quantisation conditions and Kaluza-Klein reduction, to appear in Nucl. Phys. B, hep-th/9710244.

[17] J.P. Derendinger and S. Ferrara, $N=1$ and $N=2$ supergravities coupled to matter: superhiggs effect and geometrical structure, Lectures given at Spring School of Supergravity and Supersymmetry, Trieste, Italy, Apr 4-14, 1984. Published in Supergravity 
and Supersymmetry (eds B. de Wit, P. Fayet annd P. van Nieuwenhuizen; World Scientific, Singapore, 1984).

[18] E. Bergshoeff, I.G. Koh and E. Sezgin, Coupling of Yang-Mills to $N=4, d=4$ supergravity, Phys. Lett. B155 (1985) 71.

[19] M. de Roo, Gauged N=4 matter couplings, Phys. Lett. B156 (1985) 331.

[20] J. Maharana and J.H. Schwarz, Nucl. Phys. B390 (1993) 3, hep-th/9207016.

[21] A. Sen, Strong-weak coupling duality in four-dimensional string theory, Int. J. Mod. Phys. A9 (1994) 3707, hep-th/9402002.

[22] N. Marcus and J.H. Schwarz, Nucl. Phys. B228 (1983) 145.

[23] A. Sen, Strong-weak coupling duality in three dimensional string theory, hepth/9408083.

[24] E. Cremmer, B. Julia, H. Lü and C.N. Pope, Dualisation of dualities II: Twisted self-duality of doubled fields and superdualities, to appear in Nucl. Phys. B, hepth/9806106.

[25] G. Moore, Finite in all directions, hep-th/9305139.

[26] A. Giveon, M. Porrati and E. Rabinovici, Target space duality in string theory, Phys. Rep. 244 (1994) 77, hep-th/9401139.

[27] K.S. Stelle, BPS branes in supergravity, hep-th/9803116.

[28] C.M. Hull and B. Julia, Duality and moduli spaces for time-like reductions, hepth/9803239.

[29] E. Cremmer, I.V. Lavrinenko, H. Lü, C.N. Pope, K.S. Stelle and T.A. Tran, Euclideansignature supergravities, dualities and instantons, to appear in Nucl. Phys. B, hepth/9803259.

[30] C.M. Hull, Timelike T-duality, de Sitter space, large $N$ gauge theories and topological field theory, hep-th/9806146.

[31] P.S. Aspinwall, K3 surfaces and string duality, hep-th/9611137.

[32] S.-T. Yau, Commun. Pure Appl. Math. 31 (1978) 339. 
[33] G.W. Gibbons and C.N. Pope, The positive action conjecture and asymptotically Euclidean metrics in quantum gravity, Commun. Math. Phys. 66 (1979) 267.

[34] T. Eguchi and A.J. Hanson, Asymptotically flat self-dual solutions to Euclidean gravity, Phys. Lett. B74 (1978) 249.

[35] V.A. Belinskii, G.W. Gibbons, D.N. Page and C.N. Pope, Asymptotically Euclidean Bianchi IX metrics in quantum gravity, Phys. Lett. B76 (1978) 433.

[36] S.W. Hawking and C.N. Pope, Symmetry breaking by instantons in supergravity, Nucl. Phys. B146 (1978) 381.

[37] N.J. Hitchin, private communication, reported by D.N. Page in 13].

[38] H. Lü, C.N. Pope and K.S. Stelle, Weyl group invariance and p-brane multiplets, Nucl. Phys. B476 (1996) 89, hep-th/9602140.

[39] H. Lü and C.N. Pope, p-brane solitons in maximal supergravities, Nucl. Phys. B465 (1996) 127, hep-th/9512012.

[40] M.J. Duff and C.N. Pope, Consistent truncations in Kaluza-Klein theories, Nucl. Phys. B255 (1985) 355.

[41] C.N. Pope and K.S. Stelle, Zilch currents, supersymmetry and Kaluza-Klein consistency, Phys. Lett. B198 (1987) 151.

[42] M.J. Duff, S. Ferrara, C.N. Pope and K.S. Stelle, Massive Kaluza-Klein modes and effective theories of superstring moduli, Nucl. Phys. B333 (1990) 783.

[43] M.J. Duff, J.T. Liu and R. Minasian, Eleven-dimensional origin of string-string duality: a one loop test, Nucl. Phys. B452 (1995) 261, hep-th/9506126.

[44] H. Lü, C.N. Pope, E. Sezgin and K.S. Stelle, Stainless super p-branes, Nucl. Phys. B456 (1995) 669, hep-th/9508042.

[45] H. Lü and C.N. Pope, Multi-scalar p-brane solitons, Int. J. Mod. Phys. A12 (1997) 437, hep-th/9512153.

[46] R.R. Khuri and T. Ortin, A non-supersymmetric dyonic extreme Reissner-Nordstrøm black hole, Phys. Lett. B373 (1996) 56, hep-th/9512178. 
[47] H. Lü, C.N. Pope, T.A. Tran and K.W. Xu, Classification of p-branes, NUTs, waves and intersections, Nucl. Phys. B511 (1998) 98, hep-th/9708055.

[48] R. Güven, Plane waves in effective field theories of superstrings, Phys. Lett. B191 (1987) 275.

[49] M. Cvetic and D. Youm, Class of supersymmetric solitons with naked singularities, Phys. Rev. D51 (1995) 1617.

[50] R. Kallosh and A. Linde, Exact supersymmetric massive and massless white holes, Phys. Rev. D52 (1995) 7137, hep-th/9507022.

[51] M.J. Duff, H. Lü and C.N. Pope, Heterotic phase transitions and singularities of the gauge dyonic string, Phys. Lett. B378 (1996) 101, hep-th/9603037.

[52] M.J. Duff, J.T. Liu, H. Lü and C.N. Pope, Gauge dyonic strings and their global limit, hep-th/9711089.

[53] R. Kallosh, A. Linde, T. Ortin, A. Peet and A. Van Proeyen, Supersymmetry as a cosmic censor, Phys. Rev. D46 (1992) 5278, hep-th/9205027.

[54] M.J. Duff, R.R. Khuri, R. Minasian and J. Rahmfeld, New black hole, string and membrane solutions of the four dimensional heterotic string. Nucl. Phys. B418 (1994) 195, hep-th/9311120.

[55] M.J. Duff and J. Rahmfeld, Massive string states as extremal black holes, Phys. Lett. B345 (1995) 441, hep-th/9406105.

[56] M. Cvetic and A. Tseytlin, General class of BPS saturated dyonic black holes as exact superstring solutions, Phys. Lett. B366 (1996) 95, hep-th/9510097.

[57] M. Cvetic and D. Youm, All the static spherically symmetric black holes of heterotic string on a six torus, Nucl. Phys. B472 (1996) 249, hep-th/9512127.

[58] A. Strominger, Massless black holes and conifolds in string theory, Nucl. Phys. B451 (1995) 96, hep-th/9504090.

[59] E. Bergshoeff, M. de Roo, B. de Witt, P. van Nieuwenhuizen, "Ten-dimensional maxwell-einstein supergravity, its currents, and the issue of its auxiliary fields", Nucl. Phys. B195 (1982) 97. 